\title{
SPONTANEOUS AND STTMULATED RAMAN STUDIES OF \\ VIBRATIONAL DEPHASING IN CONDENSED PHASES
}

\section{Paul Arthur Cornelius}

\section{ABSTRACT}

Vibrational dephasing in condensed phases is studied here from both a theoretical and experimental standpoint. A theory is presented which describes the dynamics of motional or exchange processes in weakly perturbed systems. This general formalism, which has been previously used to describe motlonal narrowing in magnetic resonance, is here applied to vibrational spectroscopy. The model treats the case of a high frequency vibration anharmonically coupled to a low-frequency vibration. Intermolecular exchange of low frequency vibrational quanta results in a temperature dependent broadening and frequency shift of the high frequency vibration. Analysis of experimental data by this model yields both the exchange rates and the anharmonic couplings.

The Raman spectra of $1,2,4,5$ - tetramethyl benzene (h 14 -durene) and the deuterated analog ( $\mathrm{d}_{14}$-durene) exhibit the predicted broadenings and frequency shifts. The $\mathrm{C}-\mathrm{H}$ and $\mathrm{C}-\mathrm{D}$ stretch spectra of these molecules have been taken as a function of temperature and analyzed using the exchange model. This analysis, combined with other spectral evidence, has confirmed the applicability of the model and resulted in a detailed understanding of the dephasing mecianisms which operate in this molecule. Evidence has bien cbtained pertaining both to the physical origins of the anharmonic cupplings, and to the nature of the energy t-ansfer in the low-fzzyutncy modes. 
Direct observation of vibrational dephasing in the time domain using picosecond light pulses is also described, Including experimental apparatus and techniques. The method of restricted wavevector matching Is shown to result in an estimate of the amouric of inhomogeneous broadening present in the vibrational 1in'shape. These experiments yield a data curve which is a convolution of the laser pulse shape with the vibrational correlation function, and this can be understood in terms of the theory of transient stimulated Raman scattering. Computer methods for deconvoluting the data are described which enable the measurement of dephasing times much shorter than the laser pulse width.

An understanding of the transient stimulated Raman process used In these experiments requires that the presence of self-phase modulation be taken Into account. Existing descriptions of phase modulation have been extended to radiation flelds contalning several frequency components. When phase modulation is present in multiple component fields, a pertodic progression of new frequencles is generated with a frequency splitting equal to the separation between a pair of components in the unmodulated spectrum. Such an effect is observed in methanol, which has been known to have stimulated gain in two modes. This result suggests that phase modulation may be an inherent part of the transient stimulated excitation process of moderately sized molecules.

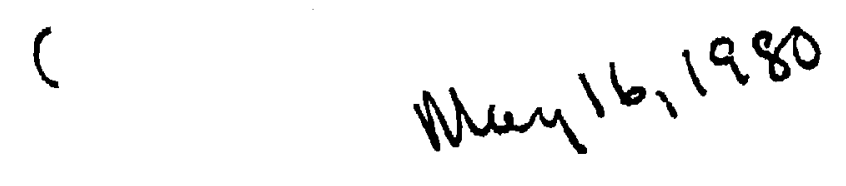


To my Parents

The beauty of things -

Is in the beholder's brain - the human mind's translation of their transhuman

Intrinsic value...so the human sense

of beauty is our metaphor of their excellence, their divine nature: - like dust in a whirlwind, making

The wild wind visible.

- Robinson Jeffers,

"The Double Axe" 
ACKNOWLEDGMENT

Behind every written account of scientific work, this is the unwritten story of the daily labors which brought the finished product to 11ght. Efficient communication of results and 1deas dictates that a colorful cast of characters be relegated to the footnotes, and that all personal debts of gratitude be only briefly acknowledged.

The largest of these debts is that owed to Bob Shelby, from whom I learned a great deal in my first few years at Berkeley. I consider myself lucky to have started graduate school with a co-worker so willing to share his knowledge, and so enjoyable to work with through thick and thin. I have had many sclentific discusstons with all of the other members of the Harris group, but spectal mention should go to Tony Gallo and Alan Campion, who were always willing to offer help and advice on any problem.

There is a long list of both friends and colleagues who deserve credit for making my stay in Berkeley both interesting and enjoyable. To name a few: Mark Lewellyn, Marc Tarrasch, Dirk Zwemer, Heinz Robota, Paul Whitmore, Mary Fullenkamp, and my neighbors Henry Luftman, LInda Young, Pauline Ho, and Alison Abbate. To all of those whose names could not be listed here: we both know who you are.

I would also like to thank the Bartlett and Connick groups for use of their Raman spectrometer. The FTIR spectra in Chapter III were taken by Linda Young and Dick MacPhatl, and the $\mathrm{d}_{14}$-durene Raman work was done by Steve Marks.

Finally, I would like to acknowledge my research director, Charles B. Harris, for financial support, for countless interesting discussions, 
and for providing a research atmosphere which makes possible the type of work described in this thesis. This work was supported in part by a grant from the National Science Foundation and by the Division of Chemical Sciences, Office of Basic Energy Sciences, U. S. Department of Energy, under contract No. W-7405-Eng-48. 
TABLE OF CONTENTS

CHAPTER

$\underline{\text { Page }}$

I INTRODUCTION .....................

II EXCHANCE THEORY. . . . . . . . . . . . 7

1. General Model of Anderson. . . . . . . . . . 7

2. Application to Vibrational Lineshapes. ...... 16

III EXCHANGE ANALYSIS OF SPONTANEOUS RAMAN SPECTRA . . . . 31

1. Other Theorles of Vibrational Dephasing. . . . . 31

2. Results and Discussion ............ 42

3. Interpretations of Exchange Theory Parameters. . . 57

4. Low-Frequency Spectra and the Exciton Mode1. . . . 72

5. Conclusions. . . . . . . . . . . . . 84

IV PICOSECOND SPECTROSCOPY. . . . . . . . . . . . 88

1. Summary of Design and Execution of Picosecond Scattering Experiments............ 89

2. Calculation of Anti-Stokes Scattering Curves . . . 113

V SELF-PHASE MODULATION IN STIMULATED RAMAN SCATTERING • 123

vi CONCLUSIONS AND PROSPECTS. . . . . . . . . . 138 APPENDIX

1 INTERMEDIATE STEP IN EXCHANGE THEORY PERIVATION. . . - 140

2 EXCHAHGE THEORY FROM ZWANZIG-MORI FORMALISM. . . . . 143

3 LISTINGS OF COMPUTER PROGRAMS. . . . . . . . . . . . 148 REFERENCES . . . . . . . . . . . . . . . . . . 167 
CHAPTER I

INTRODUCTION

One of the most fundamental processes in nature is the transfer and redistribution of energy. Everyday life provides countless examples of this, and manifestations of energy transfer on the molecular level present an equally challenging diversity, involving all molecular degrees of freedom and ambient radiation fields. In quantum systems, energy transfer is typically studied by applying a perturbation and observing the subsequent relaxation of the system back to equilibrium. The characteristic time constant for the relaxation, $T_{1}$, reveals information about the microscopic dynamics which bring about the relaxation. In addition to energy transfer, there is another process which is of equal importance, namely, the transfer of quantum-mechanical phase information. Phase transfer is illustrated most clearly in terms of an ensemble of two-level systems, in which each two-level system is represented as a harmonic oscillator. A phase-transfer event is one in which the phase of a particular oscillator is changed, but its amplitude is not affected. When such an ensemble is prepared in an initial state in which all of the oscillators are in phase, the statistical occurrence of phase-transfer events at individual sites gradually brings about random1zation of the inftially well-defined state, 1.e., the ensemble "dephases."

The combined effects of relaxation and dephasing can be described in terms of a time-dependent autocorrelation function, which is usually an exponentialjy decaying function with a time constant defined as $\mathrm{T}_{2}$. In a spectroscopic experiment, the observed lineshape is the Fourier transform of the autocorrelation function, and therefore contains contributions from both dephasing and relaxation processes. The detection and 
explanation of dephasing and relaxation processes is naturally an important area of research, not merely because of 1ts intrinsic significance, but because the study of energy dynamics necessarily involves the study of those factors which Influence it: molecular collisions, molecular structure, the perfodicity of crystal lattices, interactions between neighboring molecules in a liquid, and so on.

In a gas, the molecules spend much of their time far apart from one another. It is generally useful to consider that in the gas phase, energy and phase transfer can take place only during those isolated instants when two (or more) molecules collide. The relevant time scale is then in some way related to the collision rate. In solids and 11quids, however, a different situation exists. Every molecule is continually In close contast with Its environment, and the motions of that environment may exert a significant influence on any process that is being studied. It is not clear a prior1 to what extent the concept of a discrete, "gaslike" Interaction event may be retained when modelling such a system, nor what concepts will be useful for an elementary description of energy and phase transfer processes in condensed phases. This thesis describes an approach to one part of this question - vibrational dephasing in solids and liquids. This study has involved the development of a theoretical model, based on Intermolecular energy exchange, and the carrying out of two types of experiments: spontaneous Raman spectroscopy and picosecond laser scattering experiments.

Since vibrational dephasing times in condensed phases are typically on the order of 1-10 picoseconds, the number of experimental techniques available is 1imited. Recent advances in laser technology have made possible the generation of coherent light pulses that are a few picoseconds in length, and this has opened the possibility of studying 
vibrational dephasing and relaxation directly, f.n the time domain. The experimertal application of these pulses, which was pioneered by the Kalser group in Munich ${ }^{1-9}$ and subsequently employed in this laboratory, 10-12 allows the determination of both $T_{1}$ and $T_{2}$. Each experiment occurs in two steps: an excitation of some vibrational mode, and the subsequent probing of the excited ensemble. Although it is possible to perform the excltation step using direct infrared absorption, ${ }^{7}$ the most common technique, and the one used exclustvely in this laboratory, is stimulated Raman scattering. The probing can be done in one of two ways, elther coherently in order to measure $T_{2}$, or Incoherently in order to measure $T_{1}$.

An experiment consists of measuring the scattered signal generated In the probe process, while varying the time of arrival of the probe pulse relative to the exciting pulse. A plot of the probe scattering as a function of this time delay generates a curve which is a convolution of the probe pulse shape with the vibrational correlation function. In the case of coherent probe scattering, an important feature of the experiment is the phase-matching condition. This allows the measurement of the homogeneous dephasing time, $\mathrm{T}_{2}{ }^{*}$, removing the effects of any inhomogeneous distribution of frequencles which may be present. Comparing the dephasing time measured in this way with the linewidth of the corresponding spontaneous Raman spectrum determines the contribution of Inhomogeneities to the Raman 1ineshape.

From the theoretical standpoint, these picosecond experiments depend critically on an understanding of the transient stimulated Raman excitation process, and also on the phase-matching conditions which govern the probing process. Computer calculations, based on the theory of transient 
stimulated Raman scattering, ${ }^{6}, 10,13$ have been used to deconvolute the probe pulse shape from the experimental curves. In this way, correlation functions representing $T_{2}$ times of $<1$ psec can be measured with reasonable accuracy.

In the process of performing these experiments, it was found that the stimulated Raman spectra of some organic liquids are strongly influenced by self-phase modulation. A theory has been developed to explain these spectra, and has shown that phase-modulation is particularly 1mportant for molecules which exhibit stimulated gain in more than one mode. A particularly clear example of this has been found in the transient stimulated Raman spectrum of methanol, in which gain appears in both the symmetric and asymmetric C-H stretch modes. 14

Spontaneous Raman spectroscopy also affords a means of obtaining vibrational autocorrelation functions. In comparison with coherentprobing picosecond techniques, it offers both advantages and disadvantages. Its chlef drawback is the possible presence of inhowogeneous broadening, since it provides no means of removing or accounting for this contribution to the lineshape. On the other hand, while the picosecond experiments are limited in their application to those modes which have stimulated Raman gain, any Raman-active transition can be studied by the spontaneous technique. The ease of obtaining a Raman spectrum makes possible extensive temperature dependent studies over large regions of the spectrum, which can lead to important generalizations concerning the dynamics of the systems studied.

A large body of theoretical work currently exists which bears on the problem of interpreting spectral ifneshapes. The approaches most closely related to the theory presented in this thesis $6,7,10,12,15-29,39-59$ 
Involve the use of a stochastic description of the physical processes which influence the lineshape. Whereas much of this work has addressed questions specifically related to the probıem of vibrational dynamics in condensed phases, other treatments of energy transfer and dephasing processes $^{27-28,39-41,52-54,56}$ are also useful for understanding vibrational spectra. In particular, theories originally developed for magnetic resonance $27,28,39,40$ and extended to electronic spectroscopy ${ }^{41}$ have served as 2 gride ${ }^{15-17}$ for the theory to be discussed here.

The application of some of these Ideas to the interpretation of the temperature dependence of spontaneous Raman spectra has been the subject of extensive theoretical study in this laboratory $10,12,15-17,25$ and has generated considerable interest elsewhere. ${ }^{18-24}$ We have developed a model ${ }^{15-17}$ to describe the dephasing of high frequency $\left(3000 \mathrm{~cm}^{-1}\right.$ ) Raman transitions, which involves the presence of energy exchange in low-frequency $\left(100-500 \mathrm{~cm}^{-1}\right)$ modes. These low frequency modes are anharmonically coupled to the high frequency motions, and both the coupling mechanisms and the exchange rates can be understood using this model. The theory has been successfully applied to the C-H stretch Raman bands in $1,2,4,5$-tetramethyl benzene (durene), ${ }^{15-17}$ and to the C-D stretch bands in the deuterated form of this molecule. 25 Several other models have been advanced ${ }^{18-22}$ which account for the line broadening and frequency shift with increasing temperature that is observed in $h_{14}$-durene, but the more recent analysis of the $\mathrm{d}_{14}$-durene spectrum, combined with infrared data on both molecules, tends to support the validity of the exchange analysis. 25

The usefulness and attractiveness of exchange theory stem from its readily visualized physical basis. The paraneters which result from an 
exchange analysis of experimental data have a clearly defined physical meaning. These parameters are interesting in themselves, but more farreaching conclusions ${ }^{15-17,25}$ can be drawn by studying several modes in the same molecule, and interpreting the results in terms of a general picture of energy transfer and dephasing. Our studies on durene 25 recently demonstrated that exchange analysis can be combined with other kinds of spectral evidence to build up a quite detailed picture of the vibrational dynamics.

The organization of this thesis is as follows: in Chapter II, the exchange theory is derived, and Chapter III presents the results of our studies on the durene system. Chapter IV describes the methods of picosecond spectroscopy, and Chapter $V$ presents the analysis of the effect of self-phase modulation on stimulated Raman spectra. In Chapter VI, the Important conclusions of the work are summarized. 
CHAPTER II

EXCHANGE THEORY

This chapter presents a detalled discussion of the exchange model developed for the interpretation of temperature-dependent Raman spectroscopy. Several different derivations of the theory are possible, 17,21 , 24,26 each with some advantages of presentation over the others. For historical reasons, and for clarity in the discussion of sevcral important assumptions, the derivation based on the NMR exchange theory of Anderson will be presented here. ${ }^{27,28}$ An important alternative derivation, based on reservoir theory, is discussed in detail in References 12 and 17. It should be mentioned that these approaches differ in formal procedures only; all incorporate the same physical assumptions and approxi- . mations, and the final equations describing the Raman lineshape are the same in each case.

\section{General Model of Anderson}

We will begin by considering an isolated two-level system, whose Hamiltonian w11l be denoted $\mathcal{K}_{0}$, and whose energy splitting is $\tilde{H}_{0}$. When this system is placed into matter and is allowed to Interact with neighboring molecules, the energy levels will shift according to the effect of a "perturbation" Hamiltonian, $\mathrm{K}_{\mathrm{p}}$. As the environment of the two-level system fluctuates, the strength of the interactions comprising $\mathcal{H}_{\mathrm{p}}$ will vary in time. These motions of the environment can be represented by the "motional" Hamiltonian, $K_{m}$. The full Hamiltonian now consists of three parts (see Fig. 1):

$$
\mathscr{X}=\boldsymbol{K}_{0}+\mathscr{X}_{\mathrm{m}}+\boldsymbol{X}_{\mathrm{p}}
$$


Figure 1. An 1llustration of the three-part Hamiltonian described in the text. $\mathcal{H}_{0}$ is the Hamiltcian of the fsolated two-level system shown in (a). $\mathcal{H}_{p}$ represents the perturbation calieed by placing the two-level system into condensed matter and allowing it to finteract with its surroundings. This interaction causes a frequency shfft $\Delta \omega$ but does not broaden the Ifne (b). The motional Hamiltonian $\mathcal{H}_{\mathrm{m}}$ causes the interaction between the molecule and its surroundings to depend on time. The frequency change $\Delta w(t)$ is therefore time-dependent, and the resulting spectrun broadens. The center frequency of the spectral line will be $\omega_{0}+\Delta w_{\text {avg }}$, where $\Delta w_{\text {avg }}$ represents an average of $\Delta \omega(t)$. 


\section{GENERAL MOTIONAL HAMILTONIAN}

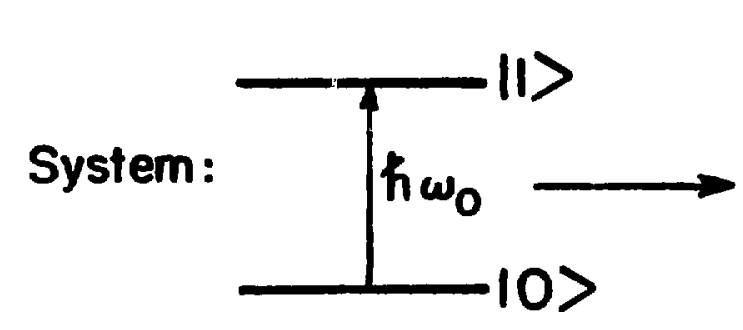

Ho

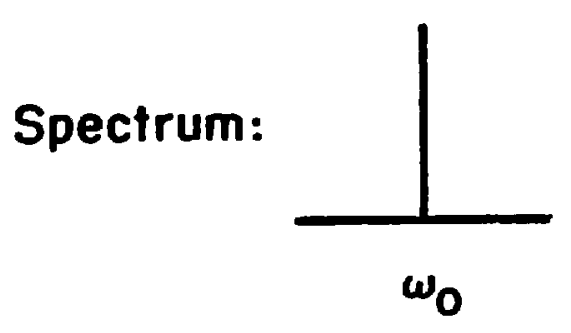

(a)

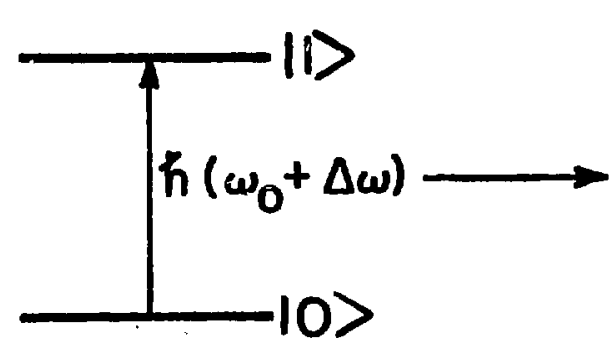

$\psi_{0}+\psi_{p}$

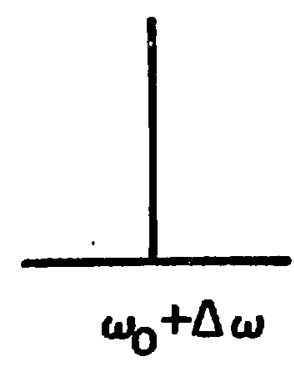

(b)

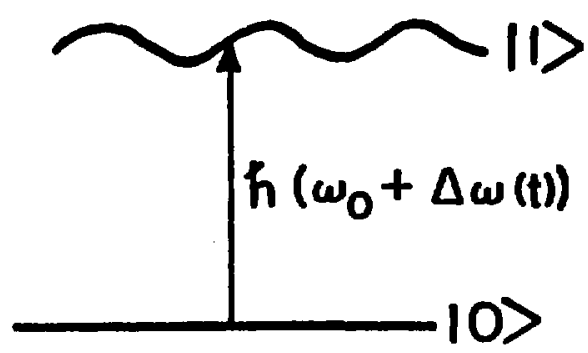

$\not_{0}+\not \psi_{p}+\not_{m}$

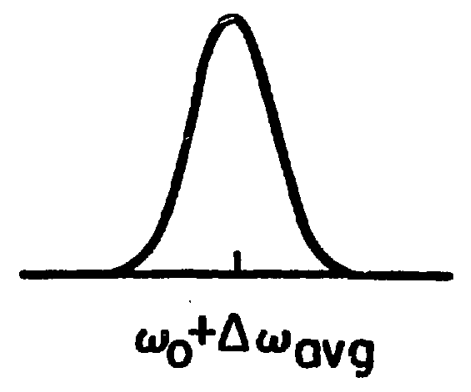

(c) 
where $\pi_{0}$ Jescribes the initial splitting, $\varkappa_{p}$ alters that splitting, and $\pi_{m}$ modifies the effect of $x_{p}$ as a function of time.

The Hamiltonian (1) is a general description of a molecule in a condensed phase, where $\pi_{0}+\pi_{p}$ determines the spectrum of the molecule In its static environment, and $\mathcal{H}_{0}+\mathcal{H}_{\mathrm{p}}+\mathcal{H}_{\mathrm{m}}$ represents the spectrum when the motions of that environment are included. From the Heisenberg equation of motion for an operator $A$ :

$$
\text { -ih } \frac{d A}{d t}=[\pi, A]
$$

we can represent the conmutation conditions which must be applied to the terms of (1) in order to describe the desired physical situation:

$$
\begin{aligned}
& {\left[x_{\mathrm{m}}, x_{\mathrm{p}}\right] \neq 0} \\
& {\left[x_{\mathrm{m}}, x_{0}\right]=0} \\
& {\left[x_{0}, x_{\mathrm{p}}\right] \neq 0}
\end{aligned}
$$

Equation ( $3 a$ ) means that the motions of the environment cause the perturbation $\pi_{p}$ to be time-dependent, whereas (3b) Indicates that those motions do not directly affect tỉe zero-order levels.

In order to calculate the spectrum, $I(\omega)$, of the system represented by (1), it is necessary to calculate the autocorrelation function of the appropriate operator - the polarizability, $\alpha$, in the case of a Raman transition:

$$
I(\omega)=\frac{1}{\sqrt{2 \pi}} \int_{-\infty}^{\infty} d t<\alpha(t) \alpha *(0)>\exp (i \omega t)
$$

The equation of motion of $\alpha$ in the interaction representation will be simply

$$
\text { in } \dot{\alpha}=\left[\mathcal{H}_{\mathrm{p}}, \alpha\right]
$$


since it is assumed that the motional Hamiltonian does not influence the spectrum directly, 1.e., $\left[\mathcal{H}_{\mathrm{m}}, \alpha\right]=0$. To compute the quantity $\langle 0|\alpha(t)| 1\rangle$, where $|0\rangle$ and $|1\rangle$ are the ground and exclted states (see FIg. 1), matrIx elements of (5) are taken to obtain

$$
\text { Ih }_{01}=\varkappa_{\mathrm{p} 00} \alpha_{01}-\alpha_{01} \mathcal{H}_{\mathrm{p} 11} \text {. }
$$

We now define a quantity $\Delta \omega_{01}$, which is the shift in resonance frequency caused by $\mathfrak{H}_{\boldsymbol{F}}$ :

$$
\Delta w_{01}=h^{-1}\left(x_{p 00}-x_{p 01}\right) .
$$

Inserting (7) into (6) gives:

$$
\dot{\alpha}_{01}=1 \Delta \omega_{01}{ }^{\alpha} 01
$$

Integration of (8) and substitution into (4) gives

$$
I(\omega)=\int_{-\infty}^{\infty} d t \exp (i \omega t) \int_{-\infty}^{\infty} d \tau \exp \left[\dot{i} \int_{\tau}^{\tau+t} \Delta \omega 0 d^{\prime}\right]
$$

The correlation function $\phi(t)=\langle\alpha(t) \alpha *(0)\rangle$ can be written as

$$
\phi(t)=\left\langle\exp \left[i \int_{t}^{t+\tau} \Delta \omega_{01} d t^{\prime}\right]\right\rangle
$$

where the brackets represent an average over all time. The time dependence of $\Delta w_{01}$ arises from the motional Hamiltonian, $\mathcal{F}_{m}$. Equation (10) is solved by choosing a particular form for $\varkappa_{p}$ and $\mathcal{H}_{m}$, thereby determining $\Delta w_{01}(t)$.

In order to make contact with the model for vibrational dephasing, we w1ll solve (10) for the case of a two-level system exchanging between 
two environments, 11lustiated in Figure 2. In environment 1, we w111 set $\Delta w_{01}=0$; in environment 2 we will set $\Delta w_{01}=\delta w$. The motional Hamlltonian will not be represented explicitly in this treatment, but w111 be assumed to cause transitions back and forth from environment 1 and 2.

At this point, two cruclal assumptions need to be introduced. The first is that the transitions between environments 1 and 2 occur adiabatically; in other words, $\mathcal{X}_{m}$ is diagonal in the base states of $\mathcal{X}_{0}$. The secular perturbation represented by $\mathcal{H}_{\mathrm{m}}$ therefore can alter the energy spacing between $|0\rangle$ and $|1\rangle$, but cannot induce transitions between them. When the two-level system changes environments, the populations of the ground and excited states will not mix, and therefore the phase of a IInear combination of $|0\rangle$ and $|1\rangle$ will not be affected. The dephasing which arises from the exchange process depicted in Figure 2 results from the presence of the frequency difference $\delta w$ in environment 2 , and not from any phase disruption in the transition from environment 1 to 2.

The second assumption is that the exchange process described by $\mathcal{H}_{\mathrm{m}}$ 1s "Markoffian." In this context, the Markoffian approximation is equivalent to saying that transitions between the two environments of Figure 2 can be completely described by a time-independent transition probability, indicated by $w_{+}$and $w_{-}$in the figure. A molecule will have a particular probability per unit time of unciergoing a transftion, regardless of how long it has been in its present environment or how it got there. This corresponds to the usual definition of a Markoffian process as one which depends only on the instantaneous state of the system, and not on its past history.

Under the assumptions of adiabaticity and Markoffian dynamics, Equation (10) can be solved for the situation depicted in Figure 2. 
Figure 2. A two-level system $|0\rangle$ and $|1\rangle$ can be placed in one of two environments. Transitione back and forth between these two environments can occur, modulating the resonance frequency of the transition. If the rates $\mathrm{w}_{+}$and $\mathrm{w}_{-}$are slow, the spectrum of an ensemble of these systems will show two discrete lines at $\omega_{0}$ and $\omega_{0}+\delta \omega$. If $w_{+}$and $w_{-}$are fast, the two lines coalasce into one, which is located in between $\omega_{0}$ and $\omega_{0}+\delta \omega$. This is the phenomenon known as exchange averaging or motional narrowing. 
EXCHANGE BETWEEN TWO ENVIRONMENTS

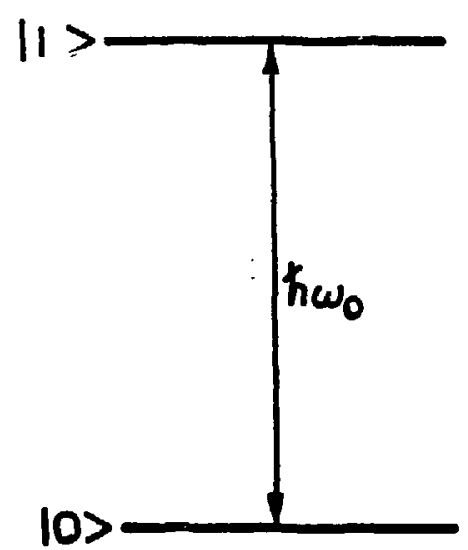

Environment 1

$\Delta \omega=0$

$$
q_{\mathrm{m}}
$$

causes transitions

from 1 to 2:

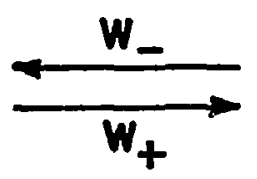

Environment 2

$\Delta \omega=\mathbf{8 \omega}$

XEL805-5125 
The solution, which is worked out in detail in Appendix 1, consists of dividing the time interval from $t$ to $t+\tau$ into $n$ equal subintervals, and requiring that $\Delta \omega$ remain constant within a single subinterval. The $n$ subintervals then represent a set of $\mathfrak{n}$ values for $\Delta w(t)$, where the probability of a glven set of $\Delta \omega$ values is determined by the transition probabilities $W_{+}$and $W_{-}$. It is assumed that ergodic behavior applies and that a statistically weighted avelaging over all sets of $\Delta \omega$ values is equivalent to the time averaging denoted by the brackets in (10). The derivation is completed by allowing $n+\infty$, and yields (Appendix 1):

$$
\phi(t)=W_{1} \cdot \exp [t(i \Delta u+\pi)] \cdot 1
$$

where ${ }_{{ }_{1}}$ is a row matrix whose elements are the equilibrtum populations in environments 1 and 2 ; $\underset{\sim}{\sim}$ is a diagonal matrix whose elements are the values of $\Delta \omega$ in the two environments, and $\pi$ is a matrix given by

$$
\underset{\sim}{\pi}=\underset{\sim}{\mathbb{W}}-\underset{\sim}{\mathbb{1}}
$$

where $\underset{\sim}{\mathbb{W}}$ is a matrix of the transition rates.

A convenient interpretation of this formalism results from considering the two-level system $|0\rangle$ and $|1\rangle$ to be a classical harmonic oscillator. A transition from environment 1 to 2 has the effect of altering the resonance frequency of the oscillator by an amount $\delta w$. If the oscillator vibrates at this shifted frequency for a length of time T and then returns to enviroment 1 , it will be out of phase by an amount $\delta \omega \tau$ with respect to an oscillator which has remained in environment 1 throughout. In an ensemble of such oscillators undergoing random transitions between environments, dephasing occurs according to the values of the parameters $W_{+}, W_{-}$and $\delta \omega$. In the case where either $\delta \omega$ or $\tau$ is very large, i.e., $\delta \omega t \gg 1$, a transition from environment 1 to 2 and back 
again represents a complete loss of phase information, and the dephasing IIfetime of the system is simply the inverse of the transition rate, $W_{+}^{-1}$. If either $\delta \omega$ or $\tau$ is very small, $1 . e ., \delta \omega \tau \ll 1$, the transition and return results in only a very silight phase shift. In this case, the dephasing lifetime will be much longer that $W_{+}^{-1}$, and the corresponding spectral lineshape will exhibit "exchange narrowing." In the intermediate regime, $\delta \omega \tau \approx 1$, the dephasing lifetime will depend sensitively on all of the parameters $W_{+}, W_{-}$and $\tau$. This is the regime of greatest interest to vibrational spectroscopy, which is the subject of the next section.

\section{Afplication to Vibrational Lineshapes}

Consider a pair of vibrational levels, the ground and first excited state of a mode whose Raman spectrum is being studied. In the harmonic oscillator approximation, and in the absence of intermolecular interactions, the Hamiltonian of this simple system can be written in terms of Its vibrational normal coordinate, $Q_{1}$ :

$$
\pi=Q_{1}^{2} \text {. }
$$

If anharmonicities are not included, higher order terms must be inserted in $\mathcal{H}$. These terms will include higher powers of $Q_{1}$ and also the coordinates of other modes:

$$
\pi=Q_{1}{ }^{2}+\sum_{j k} c_{1 j k} Q_{1} Q_{j} Q_{k}+\sum_{j k \ell} C_{1 j k \ell} Q_{1} Q_{j} Q_{k} Q_{\ell}+\ldots
$$

The effect of a given term in the anharmonic part of (14) can be calculated, to within the value of the coupling constants, using second-order perturbation theory. If the discussion is restricted to two modes, and It is further assumed that only the ground and first excited state of each are significant, the situation is illustrated in Figure 3 . The 
Figure 3. In the exchange model for vibrational dephasing, two vibrational medes - one of high frequency ( $A$ ), the other of low frequency $(B)$, couple anharmonically. The resulting level scheme is shown on the left. The resonance frequency of mode $A$ depends on the state of mode $B$; as a consequence, transitions in mode $B$ modulate the resonance frequency of $\mathrm{A}$. This situation is completely analogous to that shown in Figure 2, where the rate $W_{\text {_ }}$ can now be interpreted as the 1ifetime of the excited state of mode $B$. The energy of a quantum of mode $B$ is effectively an "activation energy" for the exchange process. 
VIBRATIONAL ENERGY EXCHANGE

BETWEEN ANHARMONICALLYY COUPLED STATES

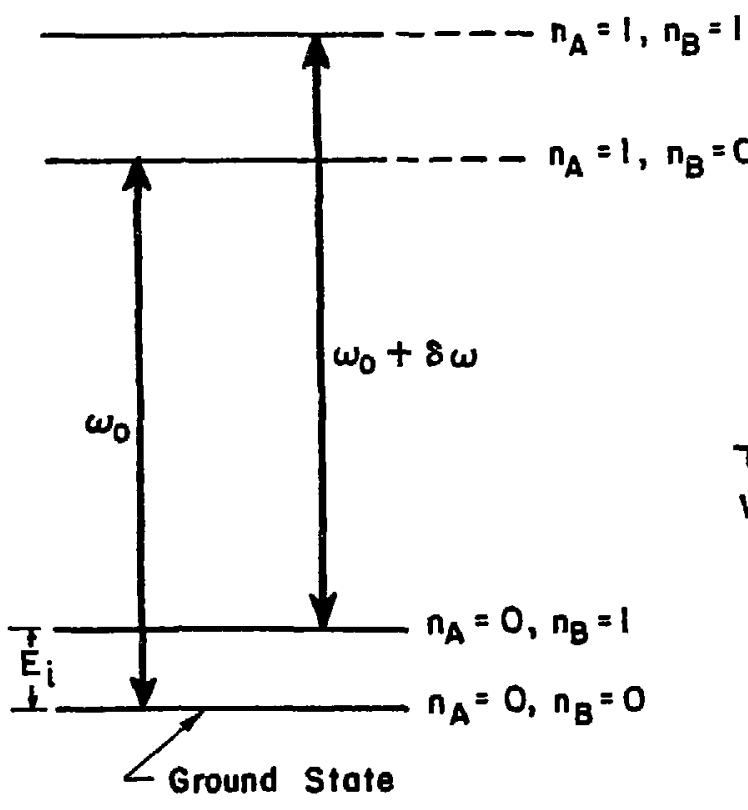

$\tau=$ Lifetime in $n_{B}=1$

$W_{+}=$Scattering from Ground Stote

Scattering Processes

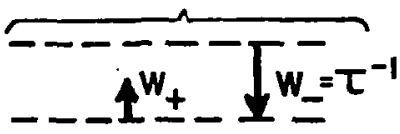

XBL 794-6107

Figure 3 
anharmonic coupling terms shift each of the vibrational levels, but the shift of the combination level $|1,1\rangle$ does not equal the sum of the shifts of $|0,1\rangle$ and $|1,0\rangle$. Thus mode 1 , the mode whose spectrum is to be studied, has a resonance frequency which depends on the state of mode 2 . Transitlons in mode 2 w11 cause the frequency of mode 1 to jump between $w$ and $w+\delta w$, In analogy with the case of two environments depicted in Figure 2 .

Table 1 illustrates the results of a perturbation calculation for typical low-order terms in the expansion of (14). The lowest-order diagonal term is of the form $Q_{1}{ }^{2} Q_{2}{ }^{2}$, and it can be expected that this term will be the dominant contribution to $\delta w$. Since no symetry resicictions apply to this term (it is always totally symmetric, regardless of the symetries of $Q_{1}$ and $\left.Q_{2}\right), 17$ any two modes cculd couple by this means.

In practice, the important case to consider is when the energy of a quantum of mode 2 is on the order of $k T$. In this case, thermal fluctuations will cause the excitation and de-excitation of mode 2 . In the subsequent discussion, it will be assumed that mode 2 is a low-energy mode $\left(100-400 \mathrm{~cm}^{-1}\right)$ and that mode 1, whose spectrum is to be calculated, is high enough in energy that 1ts exclted state is not significantly populated. A straightforward application of the Anderson formalism to this situation necessitates solving (11) for the appropriate forms of the matrices $\mathrm{W}_{1}$, $\underset{\sim}{\Delta}$, and $\underset{\sim}{\mathrm{I}}$. ${\underset{\sim}{W}}_{1}$ contains the equilibrium populations of the ground and first excited states of the low-frequency mode:

$$
\underset{\sim}{W_{I}}=\left(\begin{array}{ll}
\text { population of } & \text { population of } \\
\text { ground state } & \text { exclted state }
\end{array}\right)
$$

By detailed balance, these can be expressed in terms of the rates ${ }_{+}$ and $W_{-}$: 
Table 1. Contributions to $\delta w$ from Various Terms in the Anharmonic Potential

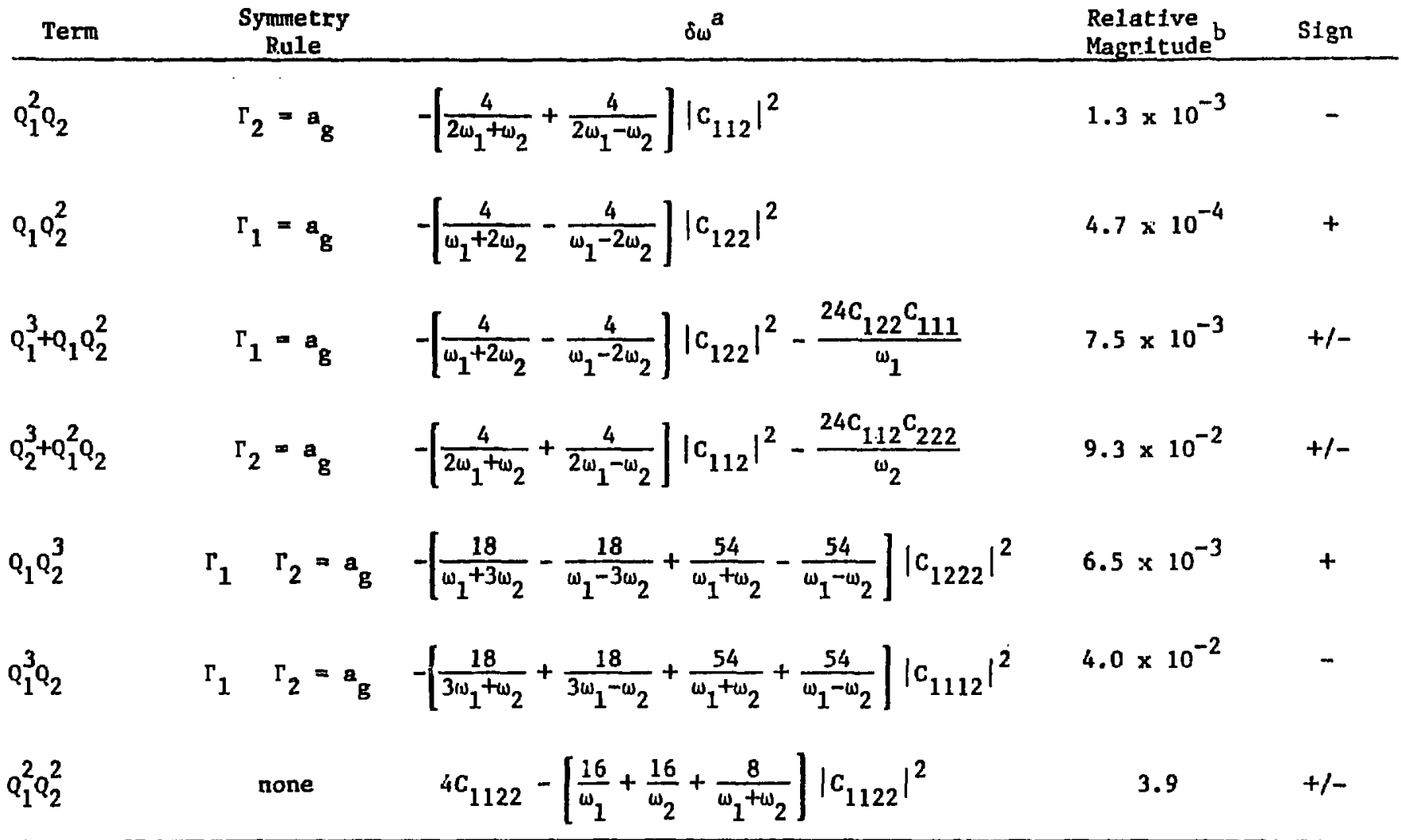

a Calculated using second order perturbation theory.

b These quantities represent $\delta \omega \mathrm{in}^{-1}$ for a situation where all c-coefficlents are equal to $1 \mathrm{~cm}^{-1}, \omega_{1}=3027 \mathrm{~cm}^{-1}$ and $\omega_{2}=263 \mathrm{~cm}^{-1}$.

ธ 


$$
w_{1}=\left(\begin{array}{cc}
\frac{W_{+} W_{-}}{1+W_{+} W_{-}} & \frac{1}{1+W_{+} W_{-}}
\end{array}\right)
$$

Since at equilibrium these populations are related by a Boltzmann factor, we can write:

$$
W_{+}=W_{-} \exp \left(-E_{1} / k T\right)
$$

where $E_{1}$ is the energy of one quantum of the low-frequency mode.

The matrix $W$ from Equation (12) is a matrix of transition rates:

$$
W=\left(\begin{array}{cc}
1-W_{+} & W_{-} \\
W_{+} & 1-W_{-}
\end{array}\right)
$$

while the matrix $\stackrel{\Delta \omega}{\sim}$ has diagonal elements only, which give the transition frequencles :

$$
\underline{\Delta \omega}=\left(\begin{array}{cc}
\omega_{0} & 0 \\
0 & \omega_{0}+\delta \omega
\end{array}\right) .
$$

Combining (12), (18), and (19) gives:

$$
1 \Delta \omega+\pi=\left(\begin{array}{cc}
1 \omega_{0}-W_{+} & w_{-} \\
w_{+} & 1\left(\omega_{0}+\delta \omega\right)-W_{-}
\end{array}\right) .
$$

Using the fact that $\phi(t)=\left\langle\alpha(t) \alpha^{\star}(0)\right\rangle$, Equations (11) and (4) can be combined to yield:

$$
\begin{aligned}
I(\omega) & =\frac{1}{\sqrt{2 \pi}} \int_{\infty}^{\infty} \exp (1 \omega t) W_{1} \cdot \exp [t(i \Delta \omega+\pi)] \cdot 1 d t \\
& =\operatorname{Re}[\underset{\sim}{W} \cdot \underset{\sim}{-1} \cdot \underset{\sim}{1}]
\end{aligned}
$$


where $A=i\left(\Delta \omega-\omega \cdot \frac{1}{\sim}\right)+\pi$. We introduce a time parameter

$$
\tau^{-1}=w_{-}
$$

which is the lifetime of mode 2, and combine (18)-(22) to give

$$
I(\omega) \alpha \frac{w_{+}(\delta \omega)^{2} /\left(1+w_{+} \tau\right)}{\left[\omega^{\prime 2}-(\delta \omega / 2)^{2}\right]+\left[\tau^{-1}\left(\omega^{\prime}+\delta \omega / 2\right)+w_{+}\left(\omega^{\prime}-\delta \omega / 2\right)\right]^{2}}
$$

where $\omega^{\prime}=\omega-\omega_{0}-\delta \omega / 2$.

This lineshape equation is plotted at three different temperatures in Figure 4. The parameters are chosen to correspond to the intermediate exchange regime $\delta \omega \tau \approx 1$, which is the case of interest for vibrational spectroscopy (see Chapter 3). As can be seen, the exchange process results in a frequency shift and a line broadening with increasing temperature. Several points should be noted concerning this figure: 1) for the particular values of the parameters chosen, the hotband $|0,1\rangle+|1,1\rangle$ transition is visible as a small shoulder on the main peak at the lowest temperature; however, it would not be visible at all if $\tau$ had been chosen to be significantly shorter. 2) The curves in Figure 4 were calculated on the assumption that $\tau$ is a temperature-independent zonstant. This assumption is expected to break down at higher temperatures. 3) The influence of higher harmonics of mode 2 is expected to become increasingly important as $\mathrm{kT}$ approaches $\mathrm{E}_{i}$. However, throughout the temperature region of experimental interest, Equation (23) is an accurate description of the exchange contribution to the lineshape.

When the frequency shift is small $\left(T+0^{\circ} \mathrm{K}\right)$, an approximate expression can be obtained from (23) by setting $\omega \cong \omega_{0}$ :

$$
I(\omega)=\left[i+\left(\omega-\omega_{\text {eff }}\right)^{2} T_{\text {eff }}{ }^{2}\right]^{-1}
$$


Figure 4. The temperature dependence of the exchange lineshape $I(\omega)$ given by Equation (23). The values of the parameters are: $\delta \omega=10.0 \mathrm{~cm}^{-1}, \tau=1.25 \mathrm{psec}, \mathrm{E}_{i}=150 \mathrm{~cm}^{-1}$. The temperatures shown are (1) $100^{\circ} \mathrm{K}$, (2) $250^{\circ} \mathrm{K}$, (3) $1000^{\circ} \mathrm{K}$. 
TEMPERATURE DEPENDENCE OF EXCHANGE LINESHAPE

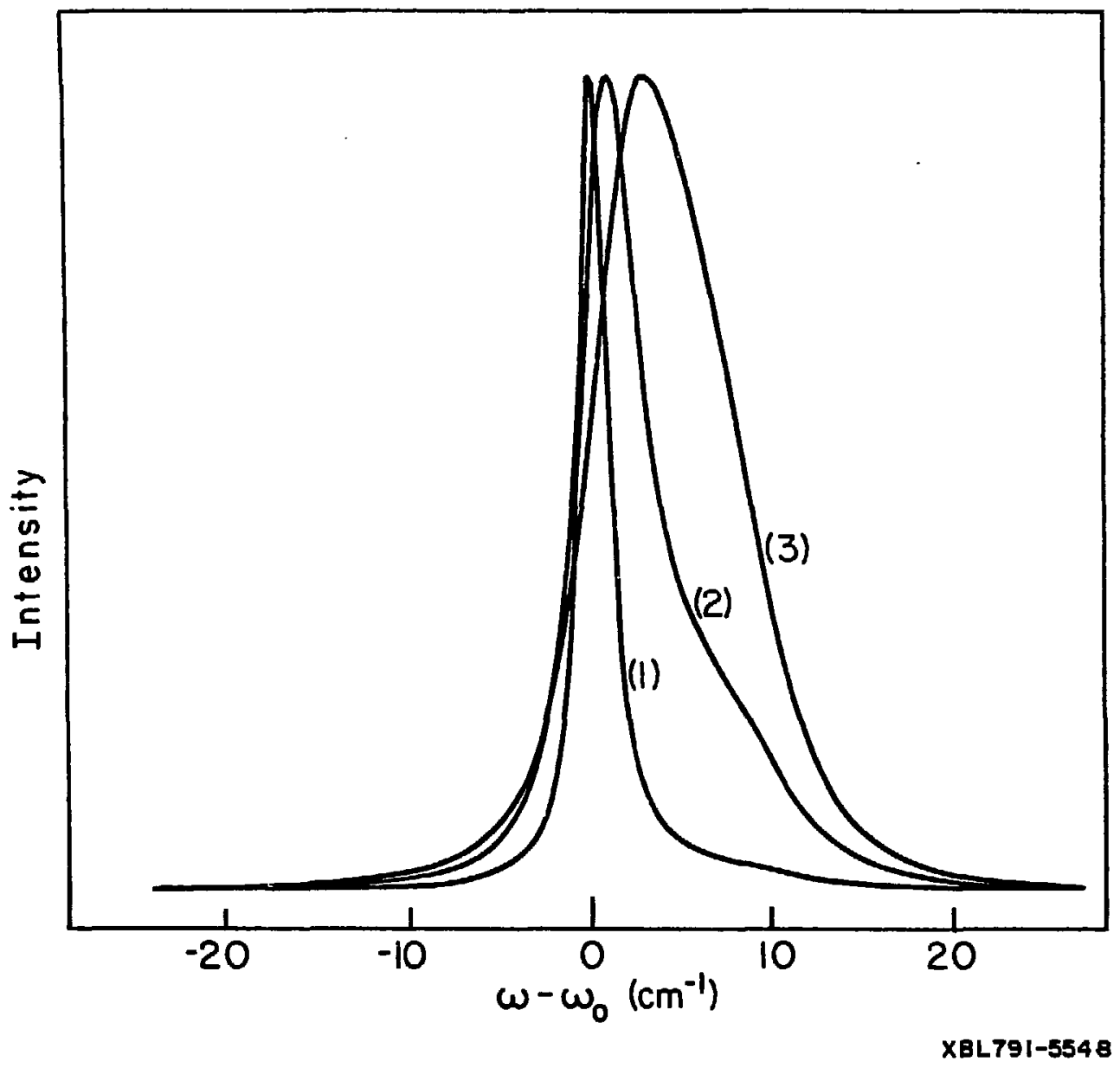

FIgure 4 
where

$$
\begin{aligned}
\omega_{\text {eff }} & =\omega_{0}+\frac{\delta \omega W_{+} \tau}{1+(\delta \omega)^{2} \tau^{2}} \\
T_{\text {eff }}^{-1} & =\frac{W_{+}(\delta \omega)^{2} \tau^{2}}{1+(\delta \omega)^{2} \tau^{2}}
\end{aligned}
$$

Equation (24) is a Lorentzian lineshape with peak position $\omega_{\text {eff }}$ and width $\mathrm{T}_{\text {eff }}^{-1}$. The width and peak position of this lineshape both vary as a function of temperature owing to the relationships (22) and (17). Substitution of these into (25) and (26) yields;

$$
\begin{aligned}
\omega_{\text {eff }} & =\omega_{0}+\frac{\delta \omega}{1+(\delta \omega)^{2} \tau^{2}}\left(\exp \left[-E_{i} / k T\right]\right) \\
T_{\text {eff }}{ }^{-1} & =\frac{(\delta \omega)^{2} \tau}{1+(\delta \omega)^{2} \tau^{2}}\left(\exp \left[-E_{i} / k T\right]\right) .
\end{aligned}
$$

These equations describe the exchange contribution to the IIneshape in the low-temperature 1imit. It is useful to represent the presence of other broadening mechanisms, which are assumed to be temperature-1ndependent, by including an additional linewidth factor, $T_{2}^{-1}$, in (28):

$$
T_{\text {eff }}^{-1}=T_{2}^{-1}+\frac{(\delta \omega)^{2} \tau^{2}}{1+(\delta \omega)^{2} \tau^{2}}\left(\exp \left[-E_{1} / k T\right]\right)
$$

Equations (27) and (29) allow graphical analysis of temperature dependent linewidths and shifts, in order to obtaln the parameters $\delta \omega$; $\tau, E_{i}, \omega_{0}$, and $T_{2}^{-1}$. The Important features of Equations (27) and (29) are as follows: 1) The peak position and width show an Arrhenius temperature dependence, $\exp \left(-E_{1} / k T\right)$, with the same "activation energy", $E_{1}$, for both the broadening and the shift. 2) In the energy level diagram 
of Figure 3, this activation energy is the energy of a quantum of the low frequency mode. 3) The second term in (29), which represents the line broadening, divided by the second term in (27), which represents the line shift, is a dimensionless constant $\delta \omega \tau$. This constant can be used to characterize the "reglme" of exchange - whether slow ( $\delta \omega \tau \ll 1)$, medium $\left(\delta_{u \tau} \approx 1\right)$, or fast $(\delta \omega \tau \gg 1)$ - and can be used to verify the assumption that $\tau$ is a constant over the temperature region of interest.

\section{Interpretation by Exchange Theory of Experimental Observations}

The hallmark of the exchange theory developed in the previous section is a broadening and frequency shifting of a vibrational lineshape with Increasing temperature. In the simple scheme thus far discussed, where the mode under ebservation is coupled to only one other mode, the broadening and stift display an Arrhenius temperature dependence, that 1s, there is an apparent activation energy for the exchange process. The activation energy for the broadening and the shift are both the same, and furthermore correspond to the energy of the fundamental of the coupled mode. Finally, the dimensionless quantity $\delta \omega \tau$ should indicate the presence of Intermediate exchange in order for this model to be applicable. If all of these conditions are met, the exchange model as discussed is a valid means of attempting to interpret the data. In this section, two points will be discussed in this connect1on: 1) the most reliable means of data analysis, and 2) under what conditions deviations from the behavior described by Equations (23), (27), and (29) may be expected.

The simplest means of data analysis is to plot the log of the line broadening and the frequency shift versus $1 / T$. Such a plot will have a slope of $-E_{1} / k$ according to Equations (27) and (29), and the intercepts 
will be given by the prefactors in these same equations. The two intercepts, one for the broadening and one for the shift, allow the determination of $\delta \omega$ and $\tau$. There are two difficulties with this procedure: the values of $\mathrm{T}_{2}^{-1}$ and $\omega_{0}$, which must be subtracted off from each point, are determined entirely from the lineshape at the lowest observed temperature, and hence are subject to experimental errors which may significantly affect the values of the parameters; also, the Equations (27) and (29) are low-temperature approximations to the exact Equation (23), and may not be valid throughout the experimental range (typically $10^{\circ} \mathrm{K}$ to room temperature).

A more satisfactory means of data analysis is to use the full lineshape expression (23), and computer programs have been developed for this purpose. Two different methods have been tried: In the first, the experimentally observed lineshapes at each temperature are digitized, and this data is then least-squares fit throughout the temperature range using Equation (23) convoluted with a temperature-independent Lorentzian line. This results in a fit of the observed temperature dependence as a function of fIve parameters: $\delta \omega, \tau, E_{1}, \omega_{0}$ and $T_{2}^{-1}$. Difficulties arise with this method when the residual low-temperature lineshape is not Lorentzian, or when two lines are close enough that they begin to overlap at high temperatures. One attempted solution to this problem, involving convolution of (23) with the observed low-temperature lineshape, proved unreliable and time-consuming. A more satisfactory approach is to use the observed widths and shifts to calculate numerically a Lorentzian lineshape, which could be subsequently run through the least squares program. This somewhat artificial procedure nevertheless has the advantage over the graphical method that the exact expression (23) is used as the basis for the temperature dependence. 
The method which was finally used as the means of analyzing the data presented in the next chapter is as follows: a program (titled LSTEMP) was developed which uses (23) to calculata the broadening and shift which result from the exchange process. This is used to perform a least-squares fit to experimental widths and shifts, using the same five adjustable parameters as above. In this case, however, only widths and peak frequencies are input into the program, which proved to be more reliable than the full lineshape procedure as well as less time-consuming. As a final check on the data, the values of $\omega_{0}$ and $T_{2}^{-1}$ obtained from this program are used to perform the graphical analysis based on (27) and (29). If the activation energies for the broadening and the fhift are the same, and agree with the value for $E_{1}$ from the least-squares analysis within experimental error, then it can reasonably be assumed the data analysis procedure is valid, and that the values of the parameters can be discussed in terms of the exchange model.

An important question concerns the interpretation of deviations from the predictions of Equations (23), (27), and (29). In developing the formalism which led to (23), the cruclal assumption was made that only two modes need to be considered: the mode under observation, and one coupled mode. In principle, a larger number of coupled modes could be accounted for by enlarglng the size of the matrices in (11) and includIng the approprlate $\delta \omega, t$ and $E_{1}$ for each additional mode. Although the problem rapidly becomes prohibitively complicated, it is illustrative to consider the effect of having two coupled modes present. A computer program developed for this purpose (named TEMDEP) has led to the followIng conclustons: 1) For certain cholces of parameters, the presence of two-mode coupling may result in an activation energy which does not match up witn the energy of either coupled mode. 2) It is also possible that 
two-mode coupling causes the activation energies for the broadening and the shift to differ considerably. 3) The ratio of the broadening to the shift is no longer expected to be temperature independent, but should still be approximately unity throughout the temperature range of practical interest.

In addition to the question of coupling to multiple modes, it is known that the quantity $\mathrm{T}_{2}^{-1}$ can be temperature-dependent. 21,22 The presence of a non-exchange, temperature-dependent contribution to the IInewidth would affect the observed activation energy for the broadening, and hence the fitted values of the exchange parameters. The importance of this effect can be evaluated experimentally in specific cases; as will be seen in the next chapter, it appears to be negligible for the cases we have studied.

In view of these considerations, it is clear that the presence of different activation energies for the broadening and the shift, a lack of correspondence between observed activation energies and known energies of the molecular modes, and a temperature dependence for the ratio of the broadening to the shift do not constitute proof of the absence of exchange. Exchange theory can readily account for such observations, although invoking a coupling to more than one mode introduces additional parameters Into the fitting process and may make the evaluation of these parameters difficult. Nevertheless, this is possible in principle.

In those cases where analysis in terms of one coupled mode is possible, the parameters obtained yield valuable information about the anharmonic couplings and energy dynamics operating in the system under investigation. In the next chapter, this analysis is carried out in detall for $1,2,4,5$-tetramethyl benzene (durene), and a thorough understanding of the vibrational dynamics of this molecule results. It is 
the possibility of this type of study which gives exchange theory Its usefulness and Importance. 
CHAPTER III

EXCHANGE ANALYSIS OF SPONTANEOUS RAMAN SPECTRA

In the previous chapter, a lineshape theory applicable to spontaneous Raman spectroscopy was developed. Several models for vibrational dephasing have been proposed by other workers as well; and in the first section of this chapter, two of those models will be discussed briefly, with a view to demonstrating how they can be tested experimentally versus the exchange theory of Chapter II.

The second section of this chapter presents experimental results of three types: the temperature dependence of the Raman spectrum of pure $\mathrm{h}_{14}$ - and pure $\mathrm{d}_{14}$-durene; the concentration dependence of the $d_{14}$-durene spectrum as it is successively diluted in $h_{14}$-durene; and the infrared spectrum of both molecules in the region where exchange theory predicts the presence of combination levels. These results are discussed in terms of exchange theory and the two models introduced in Section 1.

In Section 3, we use the parameters obtained from the exchange analysis of the data given in Section 2 to understand the vibrational interactions and dynamics of the system studied. A detailed picture results of the Importance of inter- and intramolecular couplings, resonant and nonresonant energy transfer, and delocalization of vibrational modes.

\section{Other Theories of Vibrational Dephasing}

The theory of Harris, Shelby, and Cornelius, presented In Chapter II and hereafter referred to as HSC exchange, will be used as a basis for comparing two recent models proposed by other workers: one by Abbott and $0 x$ xtoby $^{32}$ based on interactions between pairs of vibrational 
fundamentals $(\mathrm{AO})$, and the other by Wertheimer ${ }^{2 \pi}$ based on the mechanism of dynamic coupling (DC). In this section, brief explanations of these two models will be given. In parallel to the discusston of HSC exchange, each will be discussed in its simplest form where two vibrational degrees of freedom are considered.

The model of Abbott and Oxtoby (AO) is illustrated in Figure 5. The modes $A$ and $B$ are fundamentals lying close in energy, with an energy splitting of $\delta \omega$. As in HSC exchange theory above, it is assumed that the thermal activation of a low-frequency mode causes the dephasing, but here fluctuating anharmonic terms of the form:

$$
v_{\text {anh }}=f_{I I A B}(t) Q_{1}^{2} Q_{A} Q_{B}+f_{A B}(t) Q_{A} Q_{B}
$$

provide the coupling, where $A$ and $B$ are fundamentals and 1 denotes $a$ low-frequency mode.

The AO approach is similar to the formalism of Nitien and Silbey ${ }^{29}$ where the equation of motion for the matrix elements of the vibrational "superoperator" is obtained to second order in a cumulant expansion. As in the HSC case, the Markov approximation is Invoked. From this model, expressions for the frequency shifts and linewidths as a function of temperature are obtained which bear formal similarity to Equations (27) and (29) but have different physical interpretations. Assuming $\left\langle Q_{1}{ }^{2}>\right.$ and $\left\langle Q_{1}{ }^{4}\right\rangle a e^{-E_{1} / k T}$, the shift and broadening are given by:

$$
\begin{aligned}
& \text { Shift }=A \delta \omega \tau^{2} e^{-E_{I} / k T} /\left[1+\delta \omega^{2} \tau^{2}\right] \\
& \text { Width }=\text { ATe } \text { A }^{-E_{1} / k T} /\left[1+\delta \omega^{2} \tau^{2}\right] .
\end{aligned}
$$

Now $\tau$ is the decay time of the fluctuating perturbation autocorrelation function of the form: 
Figur $>$ 5. When two fundamentals differ in frequency by an amount $\delta \omega$ energy exchange between them results in dephasing. The coupling Hamiltonian involves a low-frequency mode with an energy on the order of $\mathrm{kT}$. 


\section{INTERACTING FUNDAMENTALS}

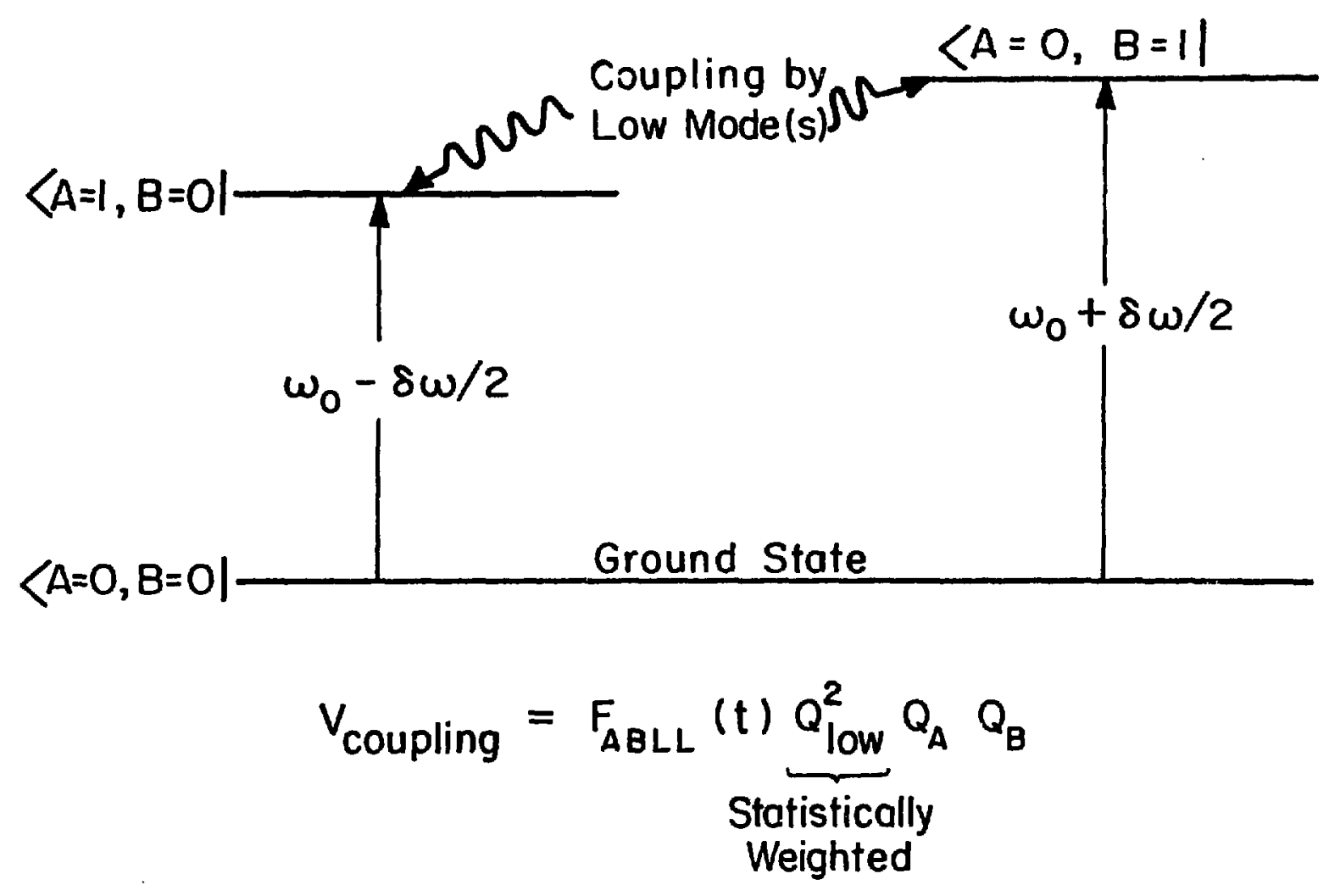




$$
<F_{\text {IIAB }}(t) F_{\text {IIAB }}(0)>a \exp (-t / \tau)
$$

where $1 \mathrm{t}$ is assumed that all the relevant decay times are the same in order to obtain the simple expressions Equations (31) and (32). Note that all the time dependence is included in $F$ and not in $Q_{1}{ }^{2}$. Thus this theory, like HSC exchange theory, predicts (in the simplest case where only two modes need be considered) an exponential temperature dependence for the broadening and shift with an activation energy equal to the energy of some low frequency mode. The following predictions also result: 1) In the specific example of Figure 5 the two 1solated fundamentals A and B should shift toward each other and display the same $E_{1}$ as the temperature is increased. 2) $\tau$ is the bath correlation time for the anharmonic force, and is typically 0.1-1.0 psec. It should also be mentioned in passing that the presence of the factor $A$ in Equations (31) and (32) makes it impossible to extract the parameters of Interest, namely $\delta \omega$ and $\tau$, as can be done in the HSC exchange model. In a recent series of papers, Wertheimer ${ }^{18-21}$ has developed a general theory of vibrational dephasing in condensed media based on the Zwanzig-Mori ${ }^{30}$ formalism. This theory 1llustrates the contributions of several mechanisms to the overall dephasing of a given transition, Including the HSC and AO exchange processes. The effects of various energy relaxation and redistribution processes, as well as cross-correlation effects, are taken into account. As was pointed out in Reference 21, the HSC exchange model of Chapter II emerges as a restricted case of the dynamic coupling theory. In Appendix 2, we discuss the nature of the approximations necessary to reduce the more general theory to this special case, and suggest how the validity of these approximations can be tested experimentally. As Appendix 2 shows, the general form of the DC theory 
introduces a dephasing mechanism based on the preence of a dispersive frequency shift $\tau^{\prime-1}$, which results from the decay of the low-frequency modes into the bath. This dispersive shift effectively brings nearly into resonance a fundamental and its anharmonically shifted hotband. Tnis results in the transfer of energy as well as phase information, and if this transfer occurs rapidly enough then one must consider the collective vibrational correlation function ${ }^{21}$ for all the modes. The importance of this contribution relative to the HSC exchange contribution depends sensitively upon the magnitudes of the various parameters, which are difficult to measure or estimate. Nevertheless, the 1ineshift and broadening should exhibit approximately Arrhenius temperature dependence, and coupling of a high frequency mode to a specific low frequency mode w111 often be observed, but the presence of additional unknown parameters makes the extraction of information from experimental data difficult.

A careful consideration of the three theoretical models suggests what experimental procedures may be employed to assess their relative applicability. In their simplest cases, the theories all predict an (approximately) exponential temperature dependence, a strong coupling to a single low-frequency mode, and an equal "activation energy" for the broadening and the frequency shift. They differ, however, in explaining the observed coupling of high and low frequency modes, and in the meanings of the frequency shift and time parameters. In Table 2 we have summarized briefly the various predictions of each model. In this section, we discuss how the differences between the theories can be related to the experiments we have performed.

In the BSC model, coupling between high-frequency and low-frequency modes is thought to result from steric interactions ${ }^{17}$ (vide infra). 
Table 2. Vibrational Dephasing Models

$\underline{\text { HSC }}$

Temperature

Dependence

Coupling

Frequency

Shift

ow

Time

Parameter,

$\tau$

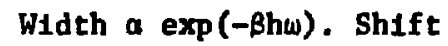
$\alpha \exp (-\beta h \omega)$. $\omega$ is frequency of the coupled low-frequency mode.

Results from anharmonic shift; dominant term is of the general form

$Q^{2} Q_{B}{ }^{2}$; suggegts steric interaction.

Sw Is anharmonic shift of combination level; may be of elther sign.

$\tau$ is resident lifetime of vibrational exciton.
$\underline{\mathrm{AO}}$

Same as HSC

Results from anharmonic term of form $Q_{1}{ }^{2} Q_{1} Q_{p}$; no specific interpretation.

ow is spacing between two adjacent fundamentals; they must shift together with increasing temperature.

$\tau$ is bath correlation time for anharmonic force constant $F_{11 A B}$. $\underline{\text { DC }}$

Very complicated, but approximately exponential as in HSC.

Results from accidental equality of dispersive shift and anharmonic shift.

Results from difference between dispersive and anharmonic shifts; may have elther sign.

Decay rates for exciton transfer processes of all types are specifically Included. 
Therefore, we would predict that isotopic substitution should not change the mode coupling scheme, 1.e., a high-frequency mode should couple to the same low-frequency mode in both the protonated and deuterated molecules. The quantity $\tau$, which can be regarded as a resident lifetime for the low-frequency vibration, should show a concentration dependence that can be Interpreted on the basis of resonant energy transfer models. The four-level exchange scheme predicts the presence of a combination level at $w_{1}+w_{2}+\delta w$ (see Figure 6). Since $\delta w$ is extracted from the analysis of the temperature-dependent 1ineshape, it is possible to compare experimental combination band frequencies with exchange theory calculated frequencies. Finally, the ratio of the width to the shift at a given temperature, for all modes studied, should yleld a temperature-independent quantity approximately equal to one.

In the AO model, two fundamentals will interact if they 1 le close together in frequency. Since the frequency spacing of the C-H stretches In $\mathrm{h}_{14}$-durene is considerably different than the C-D stretches in $\mathrm{d}_{14}$ durene, the coupling scheme may differ between the two molecules if the AO theory is correct. In elther molecule, however, two isolated fundamentals are predicted to shift toward each other with increasing temperature. Also, since the quantity $\tau$ In the AO theory is a bath correlation time, it would be expected to show at most a weak concentration dependence. In the DC mode1, couplings between modes result from a near resonance between the dispersive and the anharmonic frequency shift. Since such a resonance effect is strongly affected by small changes in either parameter, the coupling schemes in the protonated and deuterated molecules may be considerably different. The direction of shift of a given fundamental may be different in the two 1sotopic forms, and the ratio of the broadening 
Figure 6. Information about coupling of vibrational modes and vibrational dephasing of a given mode can be obtained from three different spectral regions: the fundamental region, where the mode A transition and possibly its hot band can be observed; the low frequency region, where the coupled low-frequency mode $B$ appears; and the combination band, where the peak positions can be used to confirm the presence of a coupling of mode $A$ to mode $B$. 
SPECTROSCOPY OF ANHARMONICALLY COUPLED VIBRATIONAL OSCILLATORS
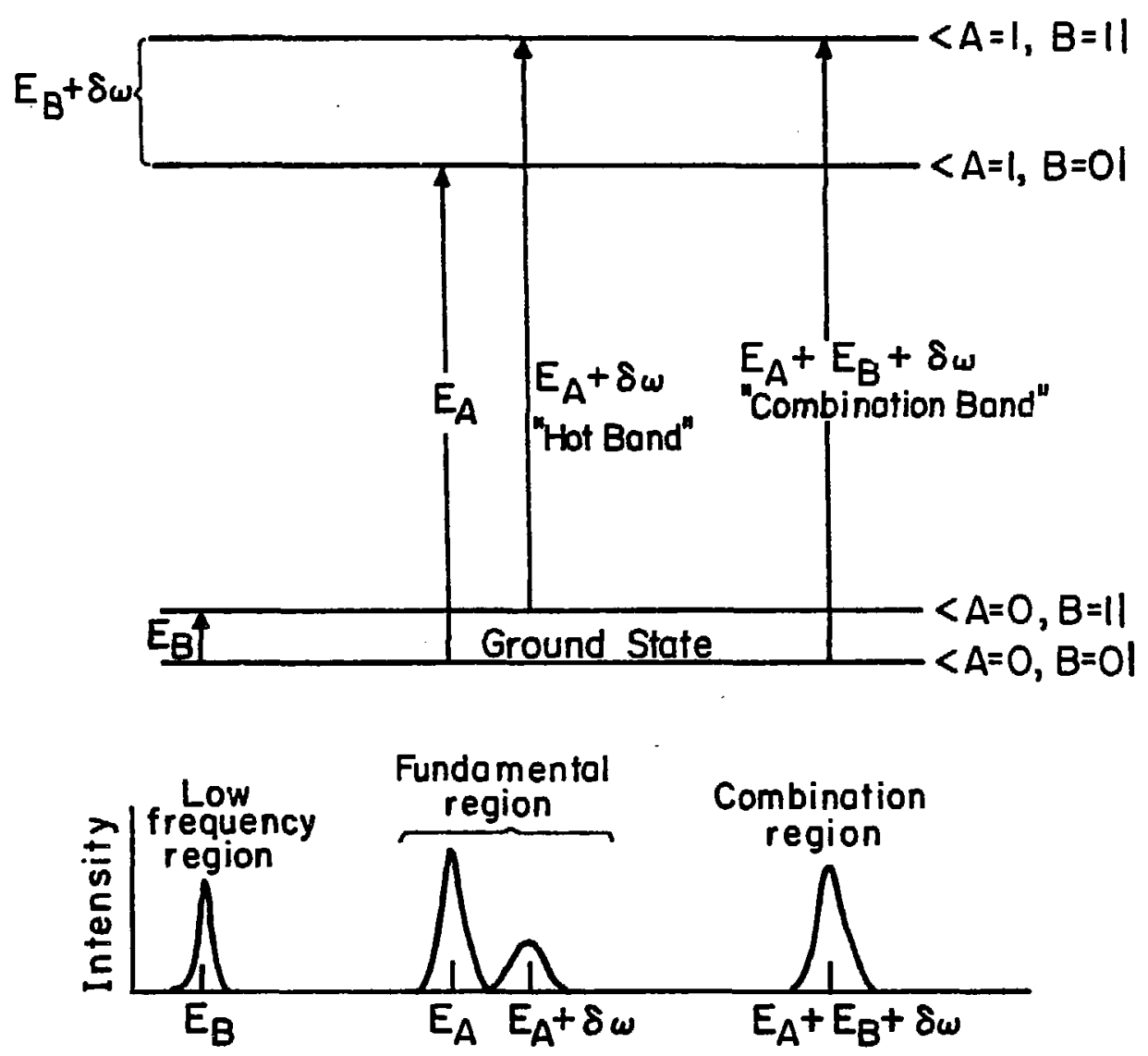

Fundomental
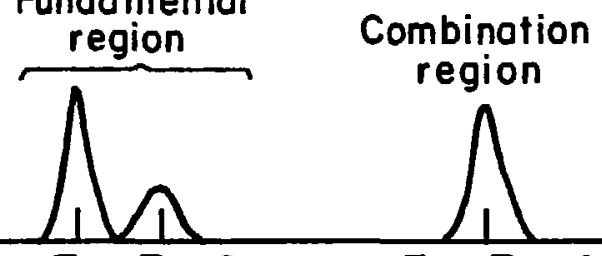
region

$E_{A} E_{A}+\delta \omega$

$E_{A}+E_{B}+\delta \omega$

Energy 
to the shift is not expected to be independent of temperature (see Appendix 2). The time parameter here is essentially the same as in the HSC model, but the anharmonic shifts are expected to be small and positive (5-10 $\mathrm{cm}^{-1}$ ). This latter prediction can be tested by observing the combination band spectrum.

Five samples of polycrystalline durene were prepared with various Isotopic compositions: pure $h_{14}$, pure $d_{14}$, and three mlxed samples with $50 \% \mathrm{~d}_{14}, 25 \% \mathrm{~d}_{14}$, and $10 \% \mathrm{~d}_{14}$ in $\mathrm{h}_{14^{\circ}}$. The samples were placed in an Air Products portable helium dewar, which could be varied in temperature from approximately $10^{\circ} \mathrm{K}$ to room temperature. The temperature was measured by a Chromel versus Gold thermocouple referenced to Ice water, which allowed temperature determination to within $¥ 1^{\circ} \mathrm{K}$ over most of the temperature range studied (slightly larger errors occur at the lowest temperatures).

The isotropic Raman spectra of the above samples were obtained in the region of interest using a Coherent CR2 Argon laser in conjunction with a Jobin-Yvon Ramanor $\mathrm{H} 625$ Raman spectrophotometer. The resolution of $1.0 \mathrm{~cm}^{-1}$ was adequate for these studies. Experimental spectra from a chart recorder were subsequently digitized using a Tektronics 4662 Digital Plotter, and computer. lineshape analysis was performed in those cases where overlapping occurred between neighboring bands. In this way, accurate values for the linewidths and peak frequencies as a function of temperature were obtained, and were then used to extract the exchange theory parameters using a least-squares fitting procedure as discussed in Chapter II. 


\section{Results and Discussion}

The Raman spectrum of $\mathrm{h}_{14}$-durene was studied as a function of temperature in the C-y stretch region from 2850-3100 $\mathrm{cm}^{-1}$. In Figure 7 , the spectra at the two extreme temperatures are shown, exhibiting five lines which strikingly manifest the broadening and shift which makes them amenable to analysis in terms of the three theorles discussed. The spectrum of $\mathrm{d}_{14}$-durene in the corresponding $\mathrm{C}-\mathrm{D}$ stretch region also shows the same type of behavior, as can be seen in Figure 8 .

Extensive computer analysis of this data, as described in Chapter IX, yields the exchange parameters in Tables 3 and 4 . Some of these parameters are meaningful only if exchange theory is valid; however, an important piece of evidence for the applicability of any of the three models is the match between the "activation energies" listed in the tables and the known energies of the low-frequency modes of the molecule. As the tables indicate, in 9 out of 12 cases the observed energy corresponds to the energy of a low-frequency fundamental. In two other cases in Table 4, the activation energy corresponds to the energy expected for a Raman-inactive combination band $\left(116 \mathrm{~cm}^{-1}\right.$ torsion plus $83.5 \mathrm{~cm}^{-1}$ 1ibration ${ }^{31}$ ).

Evidence in support of HSC exchange is obtained from the dimensionless quantity $\delta \omega \tau$, which is plotted in Figure $9 a$ and $9 b$ for the transitions studied. In both molecules, Swt is approximately unity (intermediate exchange) and is independent of temperature within experimental error. The dynamic coupling model does not, in general, predict temperature Independence for this parameter, while the HSC exchange model does assume that both $\delta \omega$ and $\tau$ are constant over the temperature region of interest. 
Figure 7. The Raman spectrum of 1,2,4,5-tetramethylbenzene at $300^{\circ} \mathrm{K}$ (top) and $11.5^{\circ} \mathrm{K}$ (bottom). The arrows indicate the direction and magnitude of the frequency shift for each line.

Figure 8. The $\mathrm{d}_{14}$-durene Raman spectrum clearly shows broadenings and shiftings characteristic of exchange. This behavior is similar to what is observed in $h_{14}$-durene. 
SPONTANEOLS RAMAN SPECTRUM: C-H STRETCHING REGION I, 2, 4, 5-TETRAMETHYL BENZENE (SOLID)

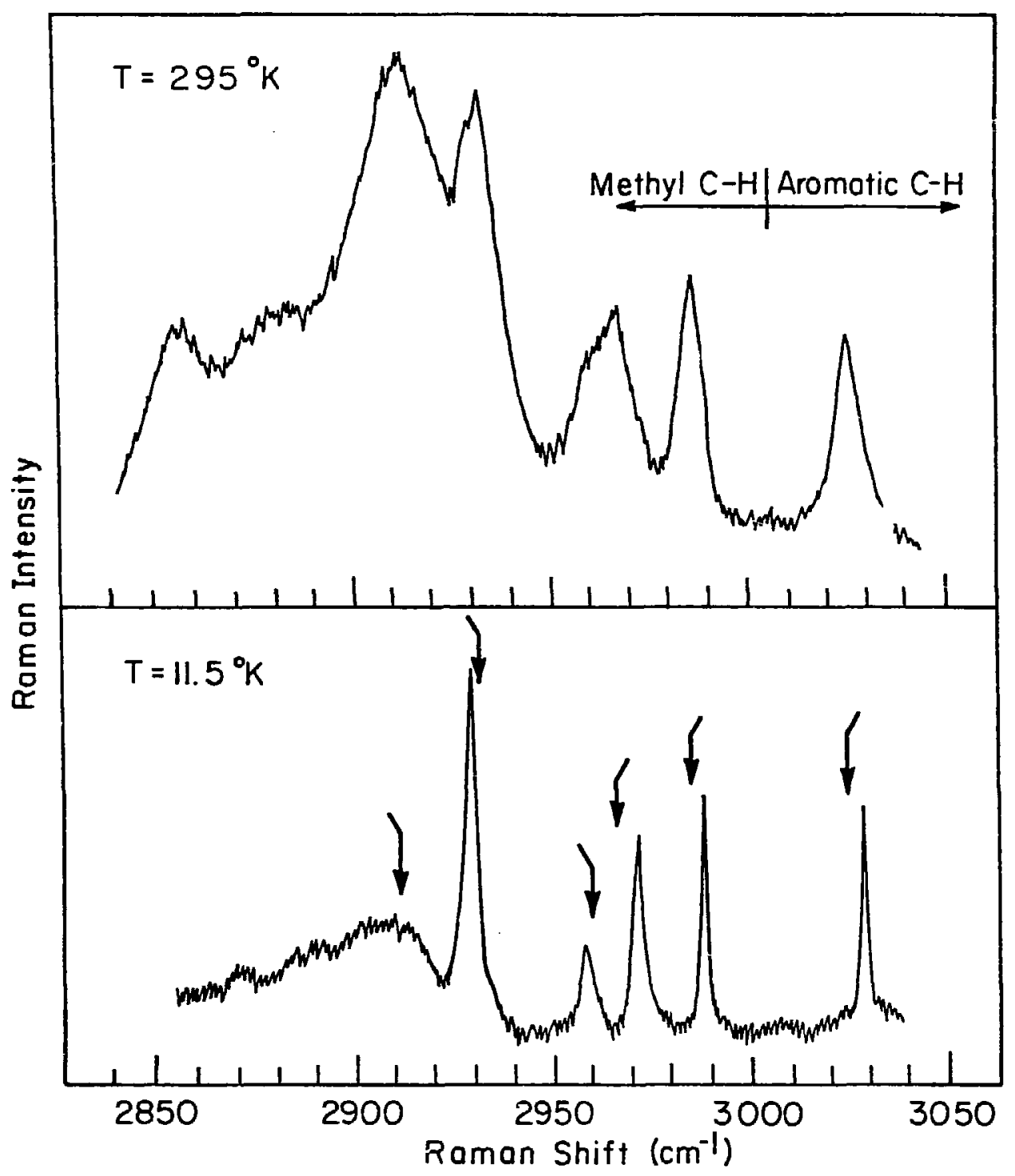

XBL $773-5179 A$ 
RAMAN SPECTRA OF $d_{14}$-DURENE C-D STRETCH REGION

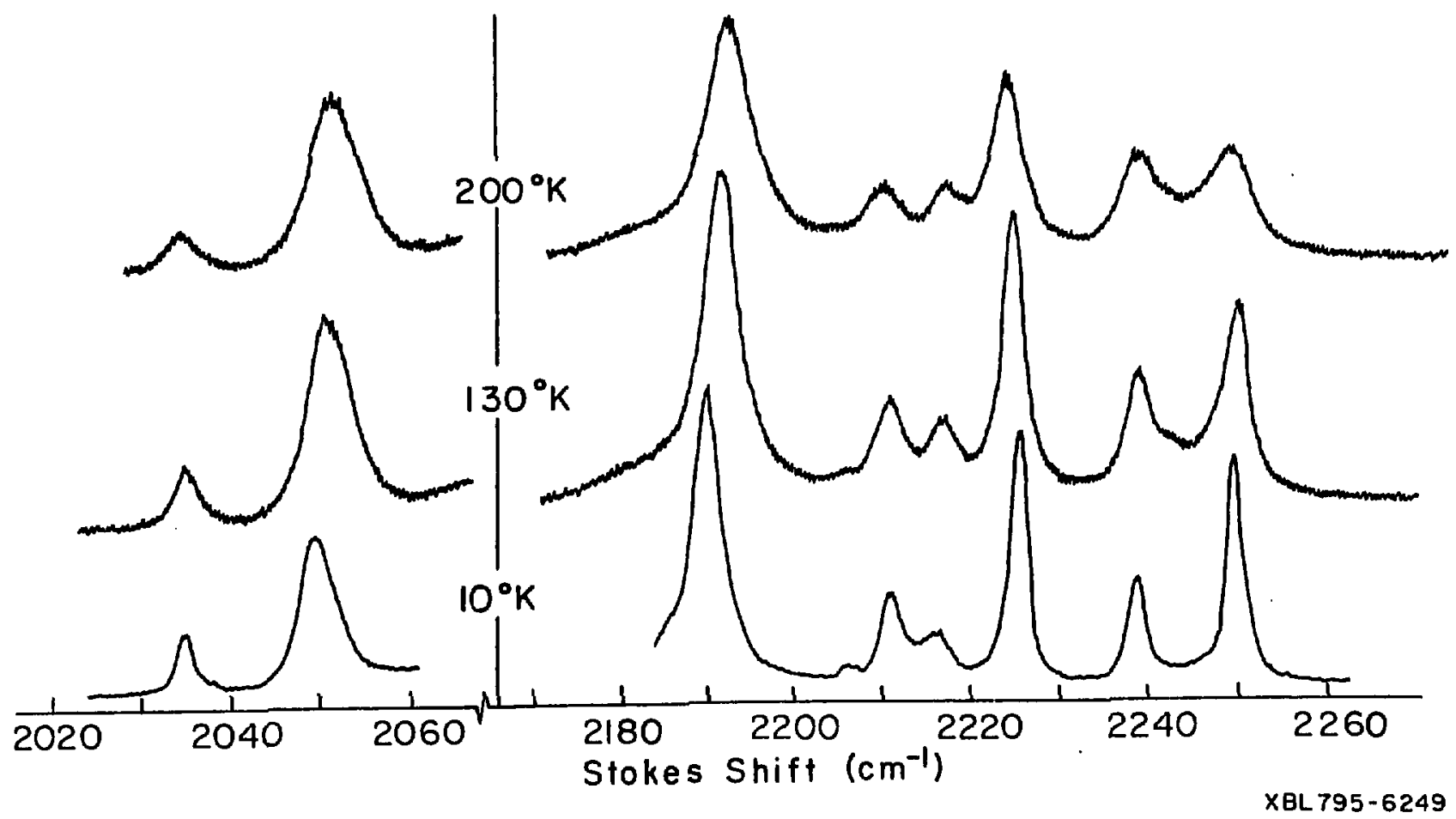

Figure 8 
Table 3. Parameters Obtained from Least-squares Analysis of Temperaturedependent Lineshape Data

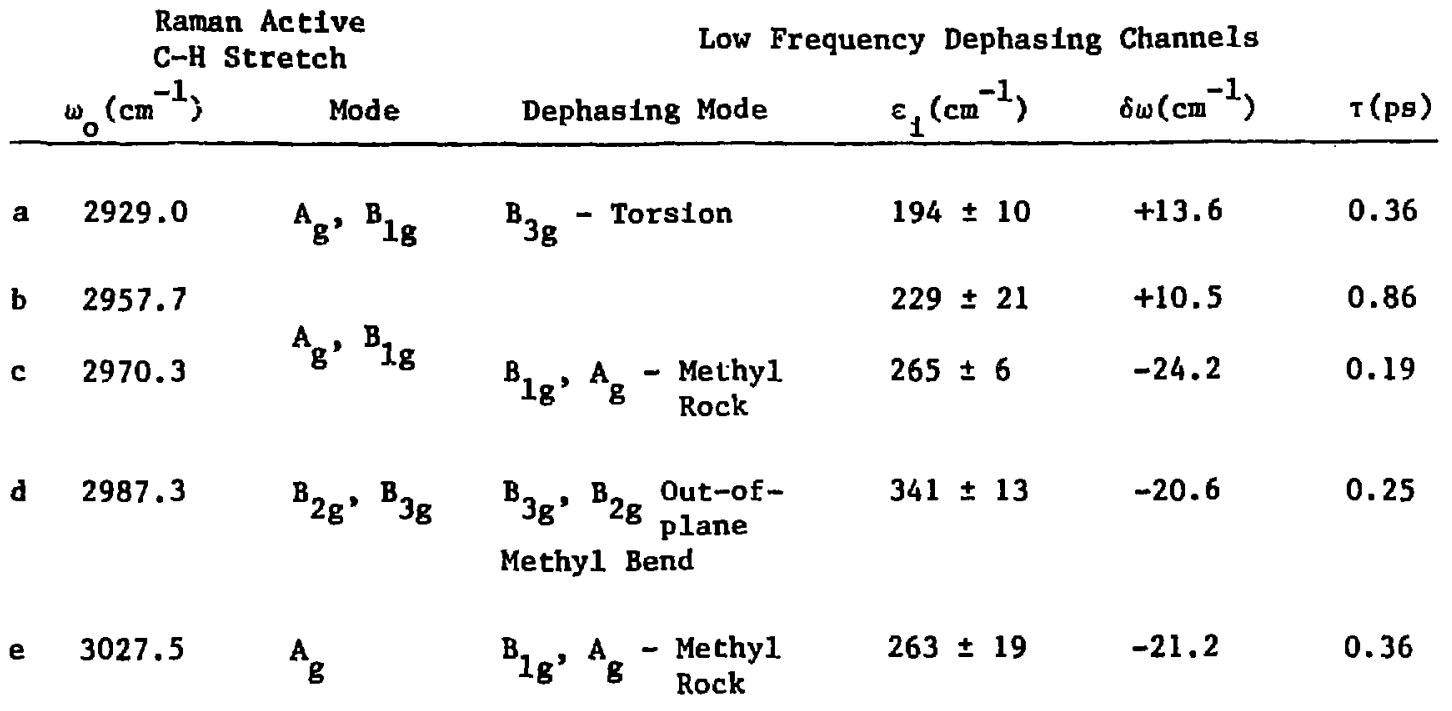


Table 4. Exchange Parameters in $d_{14}$-durene

\begin{tabular}{|c|c|c|c|c|c|c|}
\hline$\omega_{0}\left(\mathrm{~cm}^{-1}\right)$ & $\mathrm{E}_{1}\left(\mathrm{~cm}^{-1}\right)$ & Coupled low-frequency mode ${ }^{e}$ & $\delta \omega\left(\mathrm{cm}^{-1}\right)$ & $\tau($ psec $)$ & $\tau_{\text {rel }}^{a}$ & $\langle n\rangle$ \\
\hline 2035.4 & $252 \pm 10$ & $\mathrm{~B}_{1 \mathrm{~g}}$ methy1 rock & $-12.0 \pm .5$ & $.66 \pm .03$ & 2.65 & 4.02 \\
\hline 2049.9 & $190 \pm 12$ & Inactive combination band ${ }^{b}$ & $8.8 \pm .4$ & $.77 \pm .06$ & c & c \\
\hline 2191.1 & $240 \pm 20$ & $A_{g}$ methy1 rock & $19.5 \pm 1.0$ & $.30 \pm .06$ & 2.65 & 8.83 \\
\hline 2211.6 & $200 \pm 10$ & Inactive combination band ${ }^{b}$ & $-11.4 \pm .6$ & $.45 \pm .05$ & c & c \\
\hline 2225.9 & $240 \pm 15$ & $A_{8}$ methyl rock & $-16.1 \pm .7$ & $.36 \pm .07$ & 2.65 & 7.36 \\
\hline $2250.1^{d}$ & $105 \pm 10$ & & $-4.9 \pm .3$ & $3.1 \pm .3$ & 4.42 & 1.43 \\
\hline $2238.9^{d}$ & $90 \pm 15$ & & $-5.6 \pm .2$ & $4.3 \pm .2$ & 2.52 & 0.59 \\
\hline
\end{tabular}

a Calculated from the observed FWHM of the low-frequency Raman spectrum, using $\tau=1 / \pi c$ (FWHM).

b A frequency of approximately $200 \mathrm{~cm}^{-1} \mathrm{is}$ expected from a combinaticn of the torsional mode at $116 \mathrm{~cm}^{-1}$ and a libration at $83.5 \mathrm{~cm}^{-1} .36$

c The 11newidth for the coupled, Raman-Inactive combination band is not avallable.

d An additional source of error is present in these modes since the lineshapes overlap at hIgher temperatures.

e The low-frequency vibrational spectrum of $\mathrm{d}_{14}$-durene consists of a methyl rock at $240 \mathrm{~cm}^{-1}$, torstons at $116 \mathrm{~cm}^{-1}$ and $140 \mathrm{~cm}^{-1}$, and 11 brations at $83.5 \mathrm{~cm}^{-1}$ and $105 \mathrm{~cm}^{-1} .36$ 
Figure 9. The quantity $\delta \omega \tau$ is plotted as a function of temperature. The plots indicate the presence of fintermediate exchange and confirm that $\tau$ is a constant over the temperature range of the experiments. Figure $9 a$ gives the data for $\mathrm{h}_{14}$-durene; Figure $9 \mathrm{~b}$ for $\mathrm{d}_{14}$-durene. 
RATIO OF THE TEMPERATURE DEPENDENT PORTION OF THE LINEWIDTH AND FREQUENCY SHIFT IN THE RAMAN TRANSITIONS OF TETRAMETHYLBENZENE (DURENE)
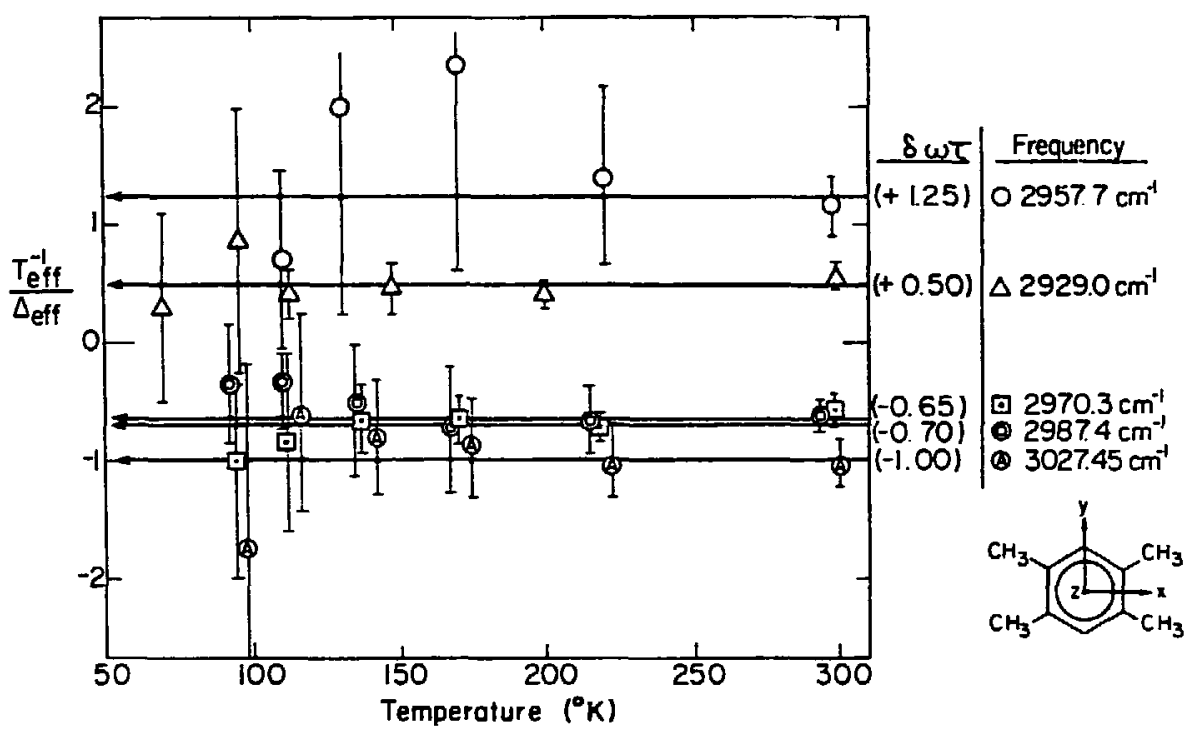

$\times 16477-58404$

Figure $9 a$ 
RATIO OF LINEBROADENING TO FREQUENCY SHIFT

AS A FUNCTION OF TEMPERATURE IN $d_{14}$-DURENE

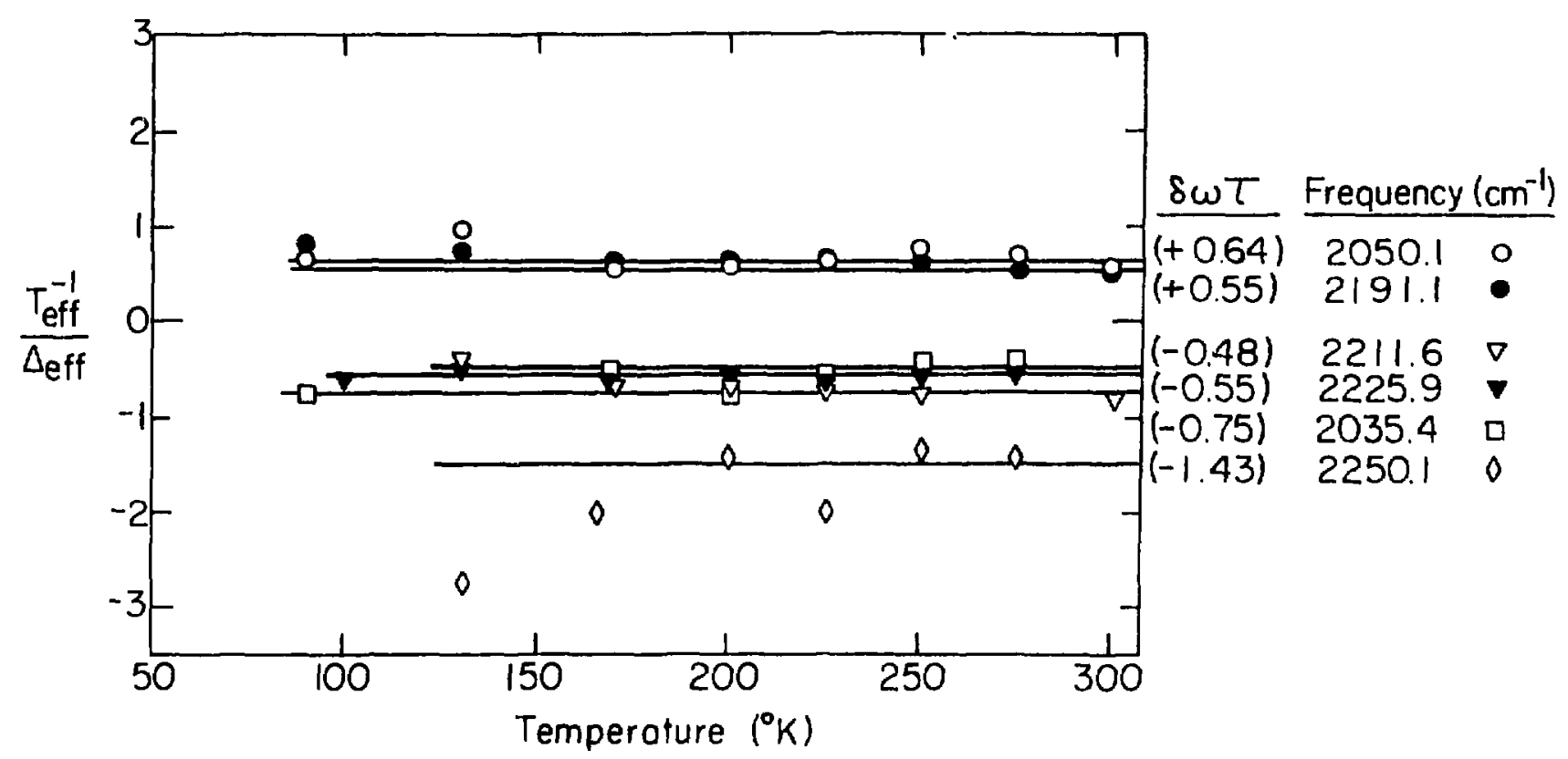

$X B L 7911-7281$

Figure 9b 
The foregoing results could also be explatned on the basis of the AO exchange model. However, the data presented in Figure 10 contradict the prediction of AO exchange that two well-separated fundamentals must shift together with increasing temperature. The figure shows two bands from the spectrum of $d_{14}$-durene; there are no Raman peaks within $100 \mathrm{~cm}^{-1}$ on the high-frequency side nor within several $100 \mathrm{~cm}^{-1}$ on the lowfrequency side. Yet the figure shows that these two peaks shift apart with increasing temperature. This could be explained by the AO model only if coupling occurs between the observed Raman peaks and some Ramaninactive transitions nearby. We have examined the IR spectrum of $\mathrm{d}_{14^{-}}$ durene in this region and found only a single peak at $2060 \mathrm{~cm}^{-1}$, and no others for at least $60 \mathrm{~cm}^{-1}$ in either direction, which indicates that the A0 model cannot account for the observed frequency shifts in Figure 10.

A further experimental test has been carried out based on a major point of distinction between HSC exchange and the DC theory, namely, the energy-level schemes used to explain the dephasing. The HSC theory predicts the preserice of combination levels which are anharmonically shifted by $\delta \omega$, whereas the DC theory predicts that the anharmonic shifts should be small and positive $\left(\sim 5 \mathrm{~cm}^{-1}\right)$. The observation of these combination bands spectroscopically therefore constitutes a crucial test of these two models. Using Fourier Transform Infrared Spectroscopy, we have successfully observed these weak bands with adequate $\left(1 \mathrm{~cm}^{-1}\right)$ resolution, and these spectra for a thin crystal of $h_{14}$-durene and for $d_{14}$-durene in $\mathrm{KBr}$ are plotted in Figures 11 and 12 respectively. The arrows on the graph indicate the peak positions predicted on the basis of exchange theory. No predictions are made for the following modes: the $2957.7 \mathrm{~cm}^{-1}$ mode In $h_{14}$-durene, which has an activation energy that does not match 
Figure 10. A portion of the Raman spectrum of $d_{14}$-durene at several temperatures. These peaks, which are clearly separated from other modes of the molecule, shift away from each other with increasing temperature, in contradiction to the $A O$ exchange model.

Figure 11. Room temperature IR spectrum of neat $h_{14}$-durene in the region where HSC theory predicts the presence of combination bands. Numbers in parenthesis are the positions of peaks predicted on the basis of the theory.

Figure 12. Room temperature IR spectrum of $\mathrm{d}_{14}$-durene in $\mathrm{KBr}$, plotted as in Figure 11. In both figures, an observed peak is located within $10 \mathrm{~cm}^{-1}$ of a predicted peak. 
FREQUENCY SHIFTS OF TWO ISOLATED BANDS

IN $d_{14}$-DURENE

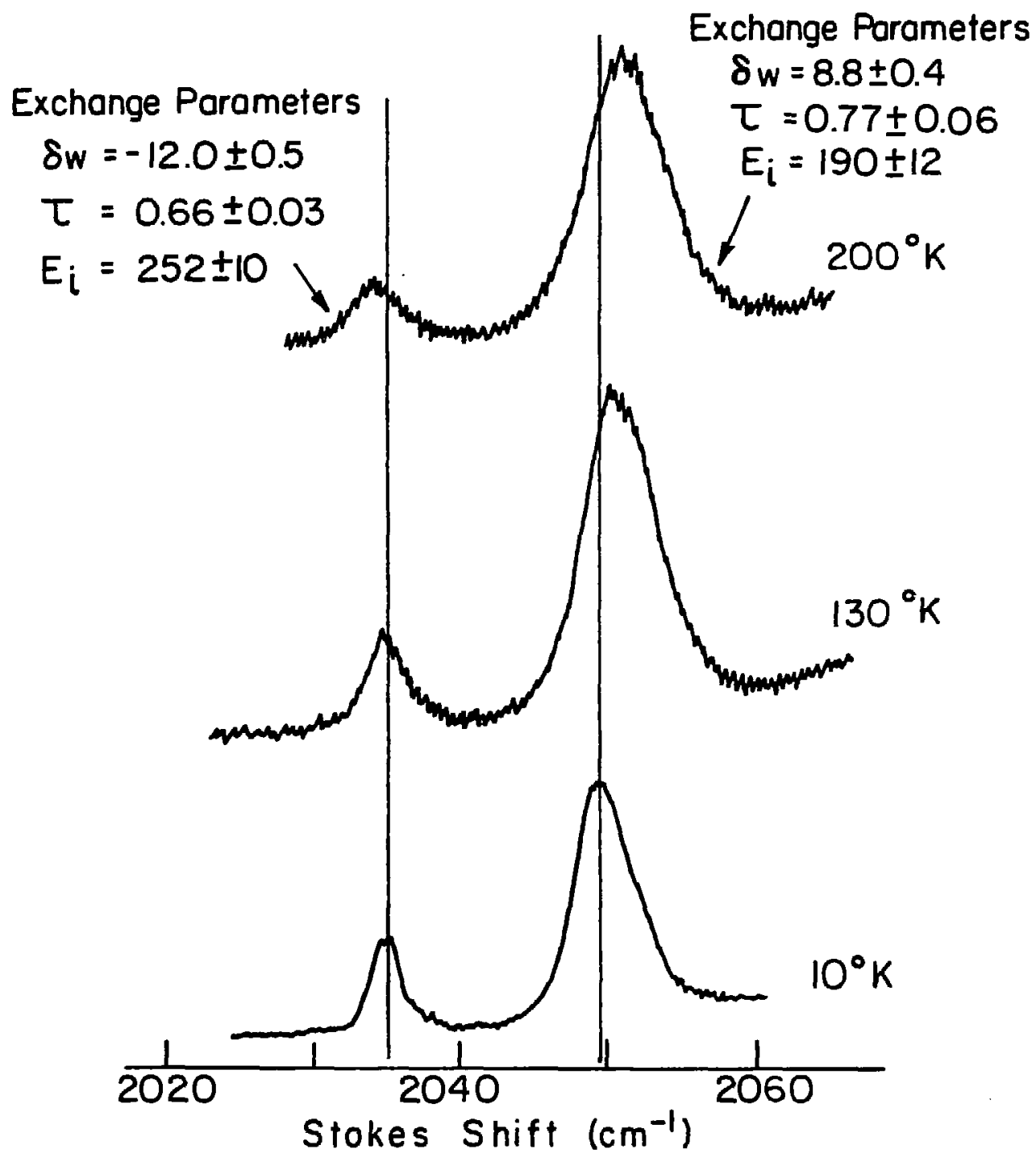

XBL7912-5432

Figure 10 


\section{FTIR SPECTRUM OF $h_{14}$ - DURENE}

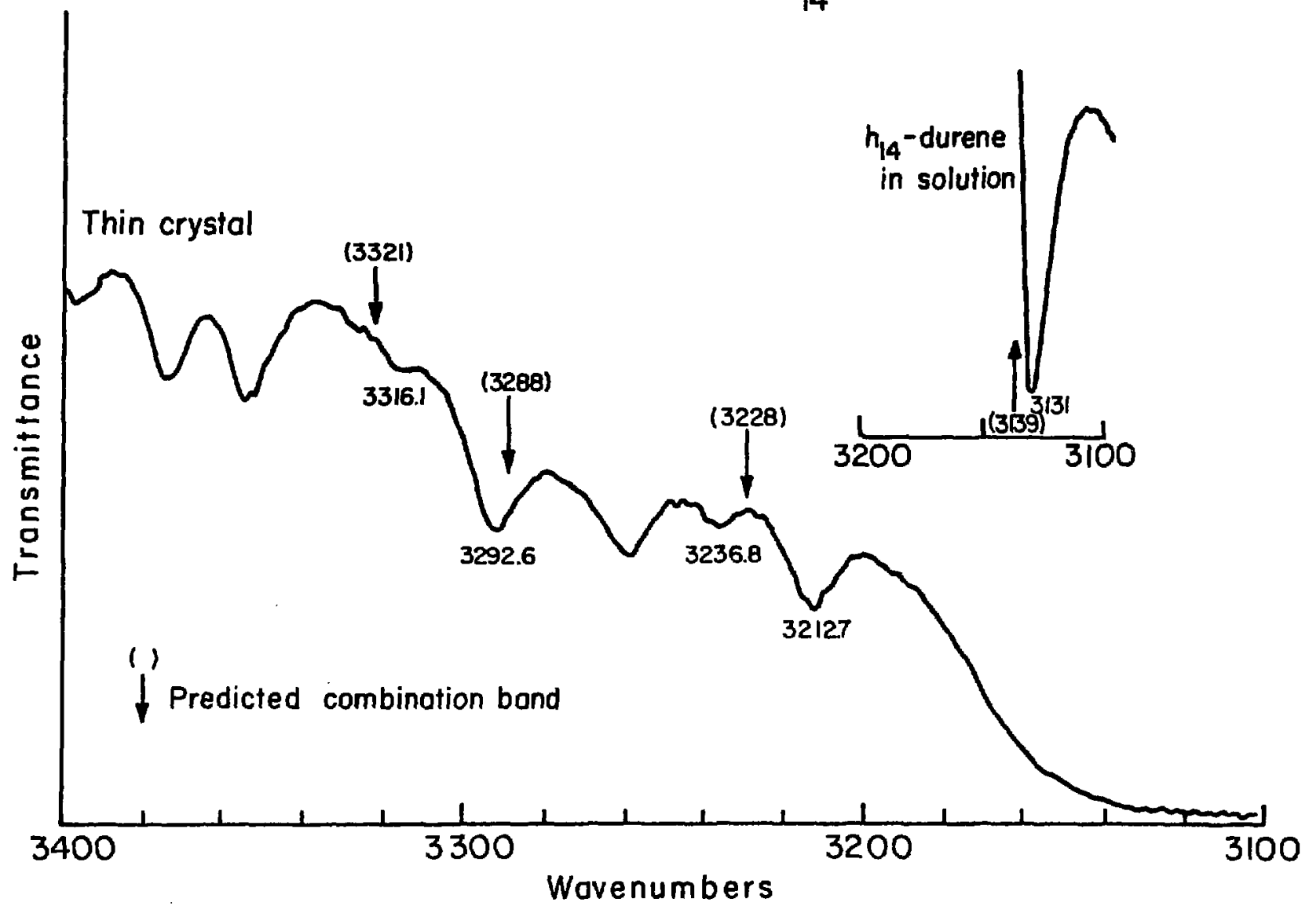

Figure 11

XBL 7912-14591 


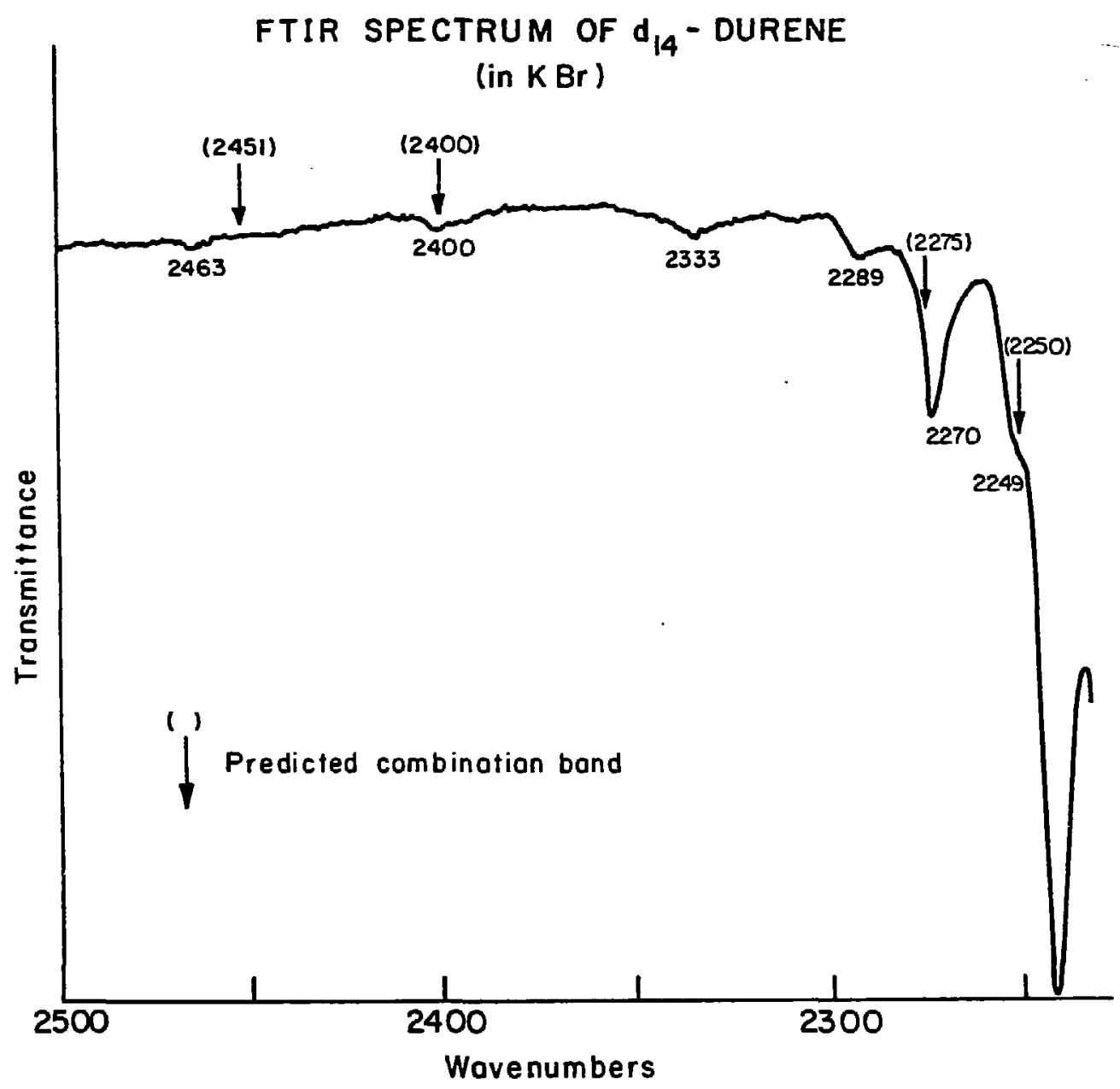

XBL 7912-14592

Figure 12 
with a low-frequency mode; the modes at $2250.1 \mathrm{~cm}^{-1}$ and $2238.9 \mathrm{~cm}^{-1}$ in $d_{14}$-durene, which are overlapping and for which it was therefore difficult to get accurate data; and the $2191.1 \mathrm{~cm}^{-1}$ mode in $\mathrm{d}_{14}$-durene, which appears to be a special case and will be discussed below in detail.

Several points should be borne in mind concerning the combination band spectra. First, the combination levels will themselves be expected to show a temperature dependence, and since the spectra were obtained at room temperature, the peaks are shifted in frequency from their zerotemperature positions by an unknown amount. Second, the spectrum of $\mathrm{d}_{14}$-durene was measured in $\mathrm{KBr}$, which contributes a matrix shift to the observed peak positions. Third, the spectrum of $\mathrm{h}_{14}$-durene in the region of interest contains a number of overlapping bands, which make accurate peak position determination difficult in some cases. We estimate that these combined effects introduce an error of $10 \mathrm{~cm}^{-1}$ or less in the peak positions. In view of this, the fact that every predicted peak falls with $10 \mathrm{~cm}^{-1}$ of an observed peak is strong evidence in favor of our model. The data is particularly convincing in the $d_{14}$ case, since the spectrum is not complicated by extra bands, and the correspondence between the predicted and observed spectra shows up clearly. We believe that this result constitutes a major piece of corroborative evidence for the exchange model.

On the basis of the evidence presented in this section, we feel Justified in retaining the simple HSC model for the purpose of analyzing the dynamics of the durene system. We recognize that the A0 exchange mechanism and the dynamic coupling mechanism must be included in any complete description of dephasing, however, our aim has been to attempt to determine the most important dephasing pathways operating in durene. All the data available can be sufficiently explained using the HSC model. 
The chief advantage of the HSC model is that it allows the ready determination of physlcally meaningful molecular parameters. These parameters are of interest in their own right, but in addition, they can be used to gain insight into those energy transfer and relaxation processes which give rise to the dephasing. In the remainder of this chapter, we assume the validity of the HSC exchange model in order to use the information which it ylelds to gain a deeper understanding of the mechanisms responsible for the dephasing in this system.

\section{Interpretations of Exchange Theory Parameters}

This section is divided into two parts: in the first, we discuss possible mechanisms for the coupling of high-frequency to low-frequency modes; in the second, we focus on how information from the low-frequency spectrum and from 1sotopic dilution experiments can be used to characterize the vibrational dynamics. Of particular interest in this connection 1s the discussion of an exciton model for energy transfer in these low frequency modes.

As discussed in the previous chapter, the fact that terms of the form $C_{11 j j} Q_{1}{ }^{2} Q_{j}{ }^{2}$ make the most significant contribution to $\delta \omega$ implies two things: $\delta w$ may be either positive or negative; and $Q_{i}$ and $Q_{j}$ may have any symmetry $\left(Q_{1}{ }^{2} Q_{j}{ }^{2}\right.$ is always totally symmetric). Any intra- or intermolecular interaction consistent with the foregoing may, in general, cause vibrational modes to couple and to exhibit a $\delta \omega$. We would like to suggest two such mechanisms which could be Invoked to explain the couplings and frequency shifts which we observe.

The most obviu.u mechanism which would couple two vibrations is a steric interaction. An illustration of this possibility in durene is shown in Figure 13. As the adjacent methyl groups torsion about the C-C 
Figure 13. These pictures illustrate the steric interaction resulting from the torsional motion of the two adjacent methyl groups. We have postulated that this steric interaction couples the torsional motion to the $2932 \mathrm{~cm}^{-1}$ methyl c-H stretch. 

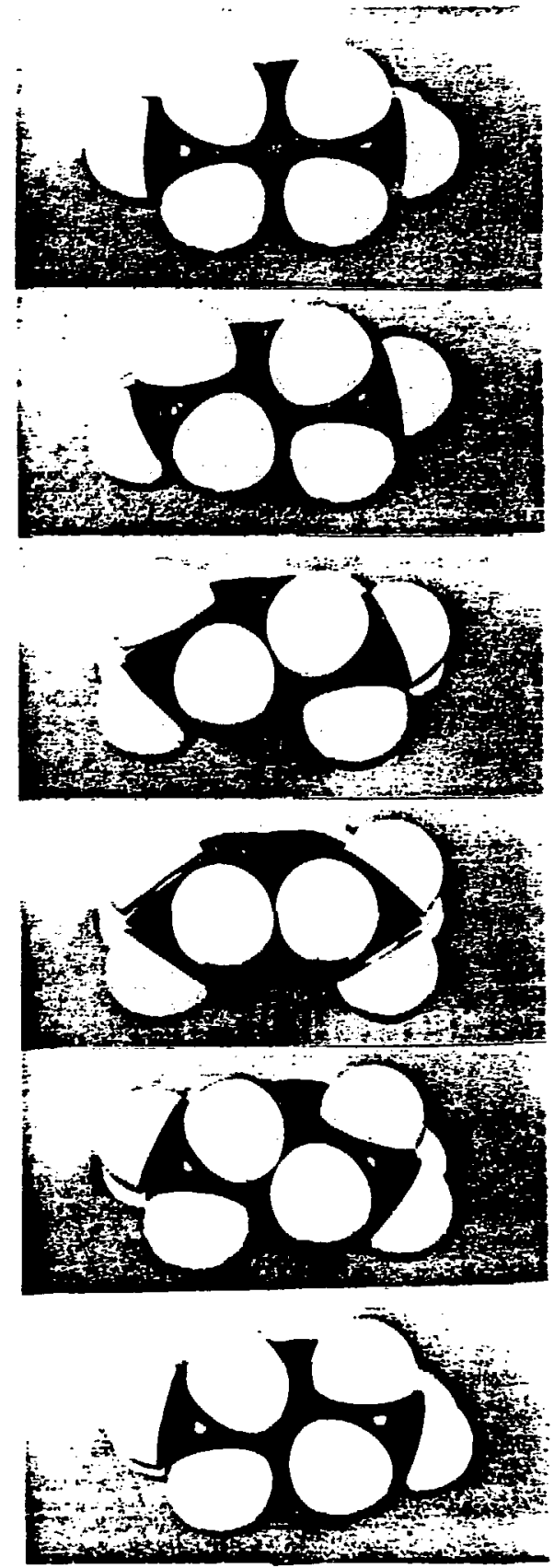

Figure 13 
bond, the nelghboring protons closely approach one another, and would be expected to interact strongly. This would evidently affect the frequency of the methyl C-H stretching modes, thus providing the type of two-mode coupling we have outlined. An energy diagram for the torsional motion is shown in Figure 14 the first excited torsional state being $197 \mathrm{~cm}^{-1}$ above the ground state. This value of $197 \mathrm{~cm}^{-1}$ should, according to exchange theory, manifest itself as an activation energy for the broadenIng and shift seen in the spectrum of the methyl C-H stretch. Our data for the C-H stretch mode at $2932 \mathrm{~cm}^{-1}$ is shown in Figures 15 and 16, and analysis of this data yields an activation energy of $194 \mathrm{~cm}^{-1}$, which agrees closely with the torsional frequency (197 $\mathrm{cm}^{-1}$, Reference 31.) The fact that $\delta w$ is positive Indicates that a torsional excitation Increases the energy of the $\mathrm{C}-\mathrm{H}$ stretch mode.

Isotopic dilution experiments carried out on $d_{14}$-durene have provided a striking plece of evidence in favor of the assumption that steric effects can be responsible for the observed couplings. Figure 17 presents the exchange parameters as a function of concentration for the $2191.1 \mathrm{~cm}^{-1} \mathrm{C}-\mathrm{D}$ stretch of $\mathrm{d}_{14}$-durene, which is coupled to the methyl rock at $240 \mathrm{~cm}^{-1}$. Both $\mathrm{E}_{1}$ and $\tau$ change significantly with concentration, which is not the case with any other mode studied. At the highest dilution $\left(10 \% d_{14}\right), E_{1}$ takes on the value of the methyl rock in $h_{14}$-durene $\left(282 \mathrm{~cm}^{-1}\right)$, and $\tau$ takes on the value measured for the methyl rock by the analysis of the $2970.3 \mathrm{~cm}^{-1}$ mode In $\mathrm{h}_{14}$-durene (see Table 3). This suggests that this high-frequency $C-D$ mode is coupled anharmonically to the methyl rocking mode on an adjacent molecule so that the exchange parameters measured characterize the molecule adjacent to the one being observed. In a highly dilute sample of $d_{14}$, the adjacent molecules are primarily 
Figure 14. Energy diagram for methyl torsion in durene. The function exhibits three-fold symetry, and the splitting between ground and excited states is $197 \mathrm{~cm}^{-1}$ (see text). 
ROTATIONAL TUNNELING IN 1,2,4,5 TETRAMETHYLBENZENE

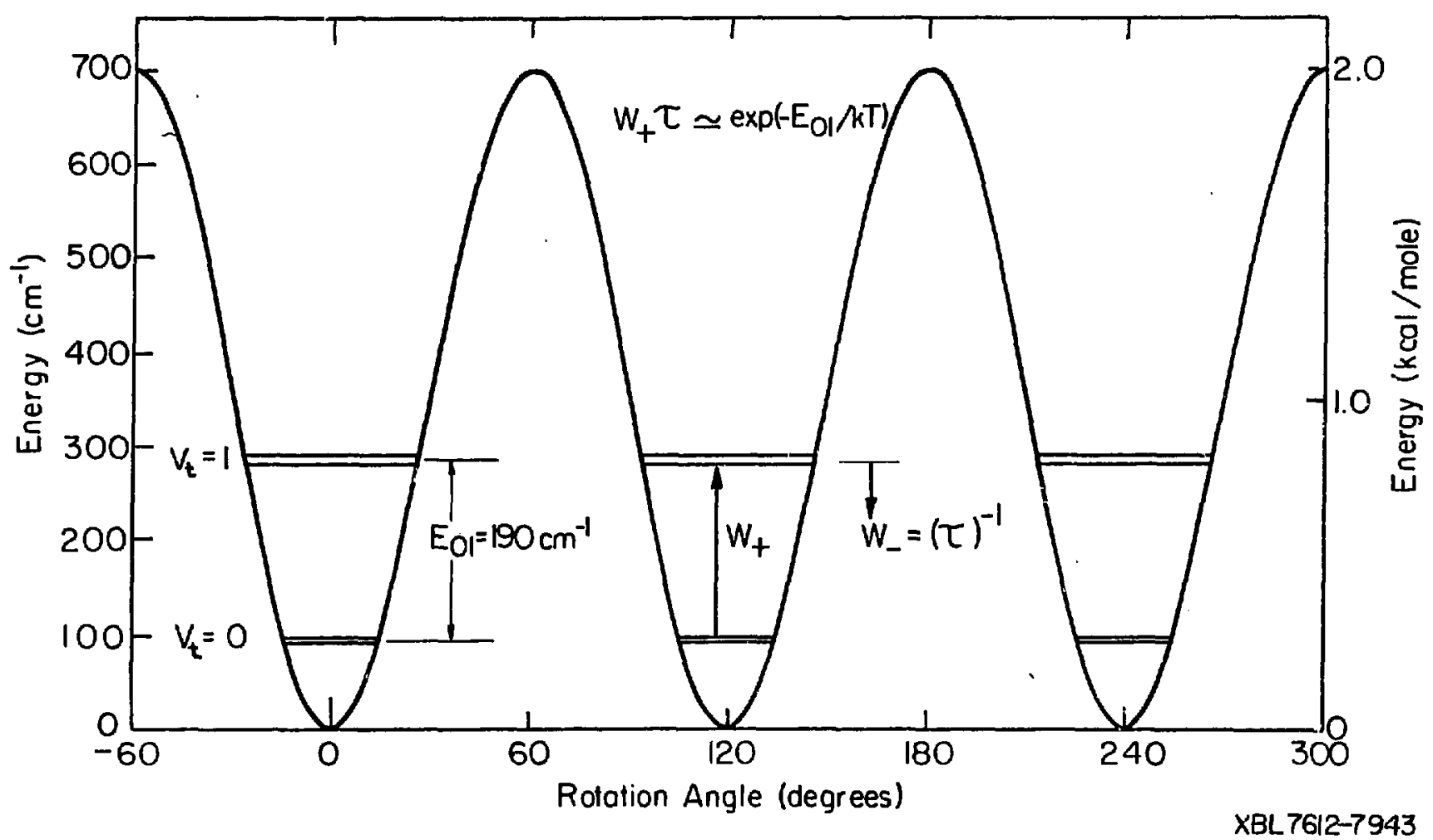

FIgure 14 
Figure 15. Linewidth vs $T$ data for the $2932 \mathrm{~cm}^{-1} \mathrm{C}-\mathrm{H}$ stretch in durene. On the left is the raw data; to obtain the straight line on the right we have subtracted a temperature independent linewidth.

Figure 16. Frequency shift vs $T$ data for the same mode as Figure 15 . Again, the raw data is on the left, and the points on the right result when the zero-temperature peak position is subtracted off. 
TEMPERATURE DEPENDENCE OF RAMAN LINEWIDTH FOR THE DURENE METHYL C-H STRETCH
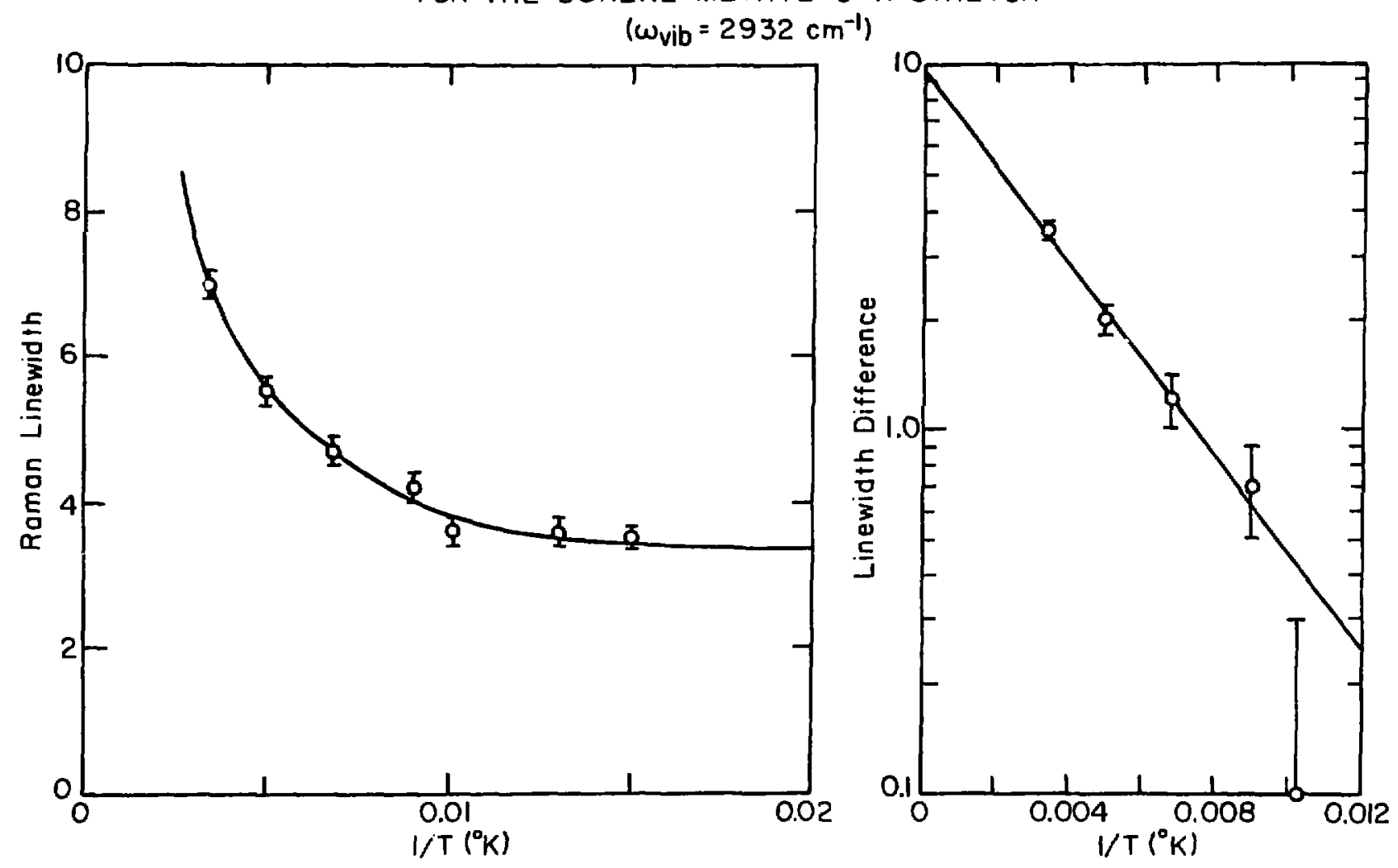

Figure 15 
TEMPERATURE DEPENDENT RAMAN FREQUENCY SHIFT FOR THE DURENE METHYL C - H STRE TCH
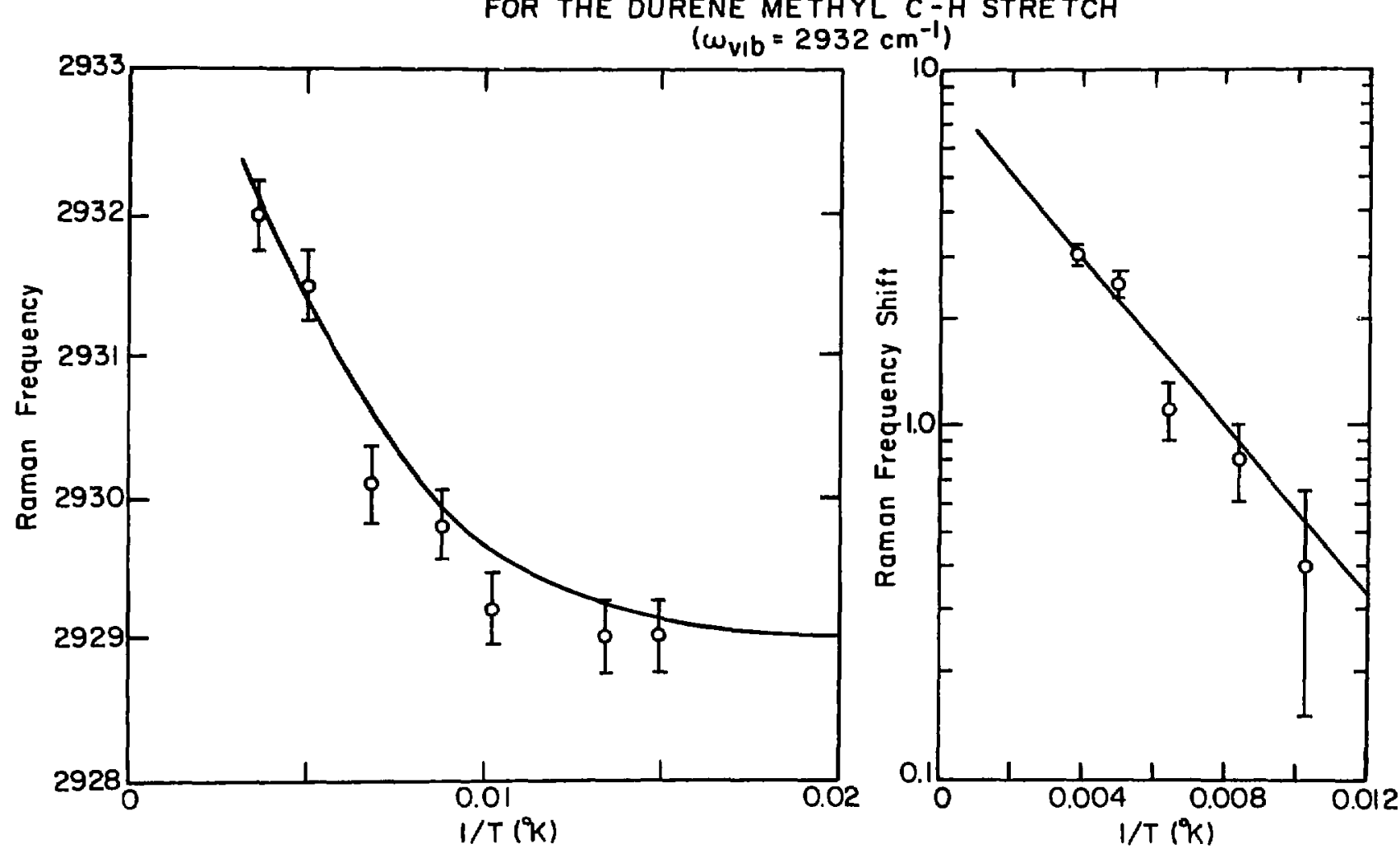

Figure 16 
Figure 17. The crystal structure of durene, showing how steric interactions between adjacent molecules can occur. The data tabulated is explained in the text by assuming that the $2191 \mathrm{~cm}^{-1} \mathrm{C}-\mathrm{D}$ stretch is coupled to the methyl rock on the adjacent molecule. 
INTERMOLECULAR VIBRATIONAL DEPHASING IN DURENE

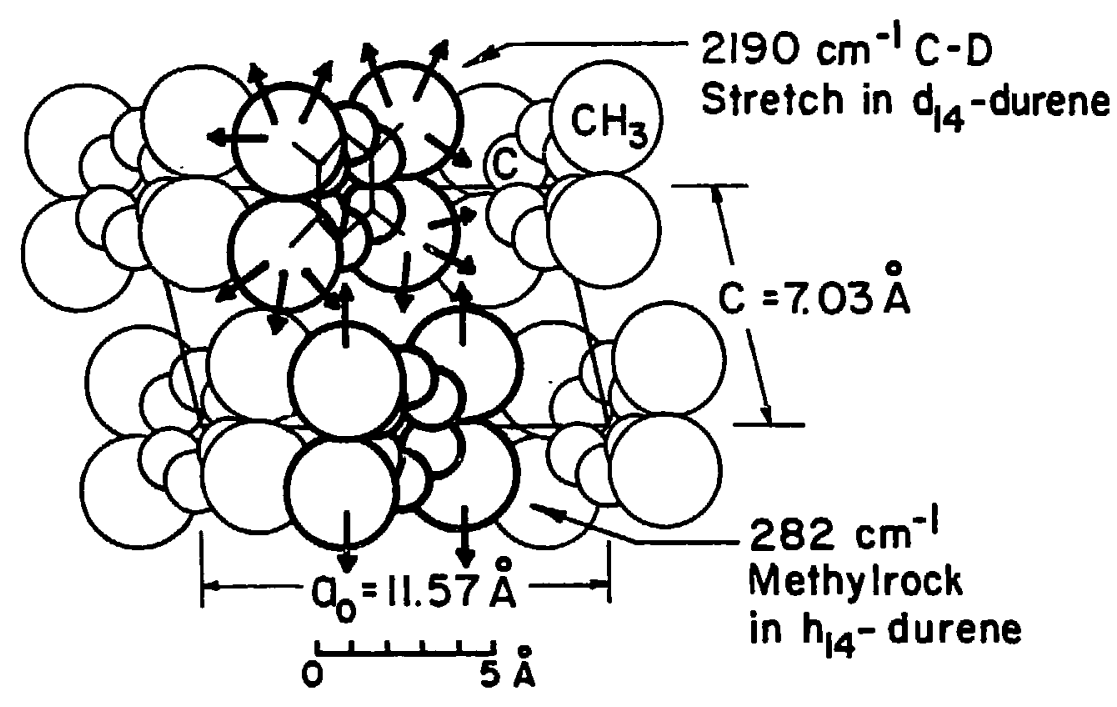

$2190 \mathrm{~cm}^{-1} \mathrm{C}-\mathrm{D}$ Stretch of $D_{14}$-Durene [Methyl D's]

$\begin{array}{lllll}\frac{\text { Environment }}{100 \% d_{14} \text {-durene }} & \frac{\delta w\left(\mathrm{~cm}^{-1}\right)}{19.5 \pm 1} & \frac{E_{i}\left(\mathrm{~cm}^{-1}\right)}{240 \pm 10} & \frac{\tau(p \mathrm{sec})}{0.30 \pm .05} \\ \begin{array}{l}50 \% d_{14} \text {-durene } \\ 50 \% \mathrm{~h}_{14} \text {-durene }\end{array} & 21.1 \pm .5 & 260 \pm 10 & 0.24 \pm .03 \\ 25 \% d_{14} \text {-durene } & 22.4 \pm .5 & 280 \pm 10 & 0.21 \pm .03 \\ 75 \% \mathrm{~h}_{14} \text {-durene } & & & \\ 10 \% d_{14} \text {-durene } & 21.6 \pm .3 & 285 \pm 10 & 0.19 \pm .03 \\ 90 \% \mathrm{~h}_{14} \text {-durene } & 21.6 \pm & & \end{array}$
XBL オ $\because 83$ 
$h_{14}$, so $E_{i}$ and $t$ approach their values for $h_{14}$-durene as dilution increases. Figure 17 shows the crystal structure of durene, and 11lustrates how this proposed intermolecular steric interaction takes place. It can reasonably be assumed that the $2970.3 \mathrm{~cm}^{-1}$ mode in $\mathrm{h}_{14}$-durene is coupled by a similar mechanism, and that a general understanding of the nature of the stertc couplings in a given molecule may require knowledge of the crystal structure as well as the geometry of the isolated molecule.

In a substituted benzene molecule, the electronegativity of the substituents strongly affects the electron density in the ring. The vibrational frequency of a proton attached to a substituted benzene ring depends on the distribution of electron density within the ring, and hence on the nature and location of the substituents. This fact suggests a second mechanism which may be responsible for coupling the vif a nal modes in durene; however, owing to the difficulty of calculating electron densities in vibrationally excited molecules, the following discussion is necessarfly speculative. It is reasonable that the exctation of a vibrational mode in a molecule such as durene would significantly perturb the electron distribution in the ring. This would then affect the frequencies of other modes, and, it should be noted, could shift them to efther lower or higher energies, depending on the specifics of the situation. In durene, one would expect this mechanism to manifest itself most strongly in the case of the aromatic proton stretch, since these protons are in close contact with the ring.

In Figures 18-19 we show our data for the aromatic $\mathrm{C}-\mathrm{H}$ stretch mode In durene $\left(3025 \mathrm{~cm}^{-1}\right)$. Again, the temperature dependence exhibits the qualitative behavior suggestive of exchange, and a careful analysis yields a $\delta \omega$ of $-21.2 \mathrm{~cm}^{-1}$ and an activation energy of $263 \mathrm{~cm}^{-1} \pm 19$. 
Figure 18. Linewidth vs $\mathrm{T}$ for the $3025 \mathrm{~cm}^{-1} \mathrm{C}-\mathrm{H}$ stretch in $\mathrm{h}_{14}$-durene. The plot is arrived at in the same way as Figures 15 and 16, where the points on the right were obtained from the raw data on the left by subtracting off a temperatureIndependent linewidth.

Figure 19. Frequency shift vs $T$ for the same $3025 \mathrm{~cm}^{-1}$ mode. The zero-temperature peak position was subtracted from the raw data on the left to give the straight line on the right. 
TEMPERATURE DEPENDENCE OF RAMAN LINEWIDTH FOR THE DURENE AROMATIC C-H STRETCH

$\left(\omega_{\text {vib }}=3025 \mathrm{~cm}^{-1}\right)$
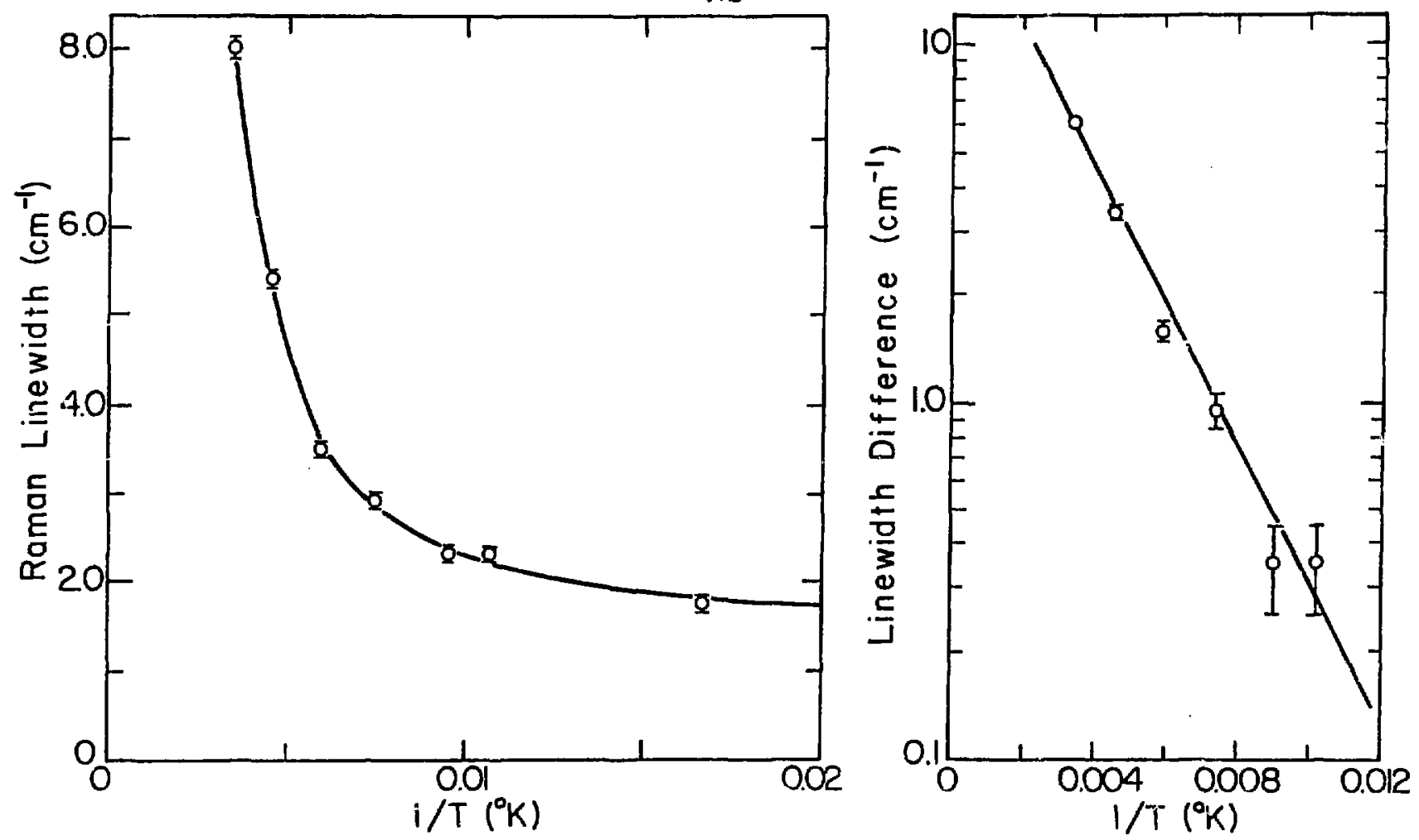

Figure 18

XBL $791-5549$ 
TEMPERATURE DEPENDENT RAMAN FREQUENCY SHIFT FOR THE DURENE AROMATIC C-H STRETCH

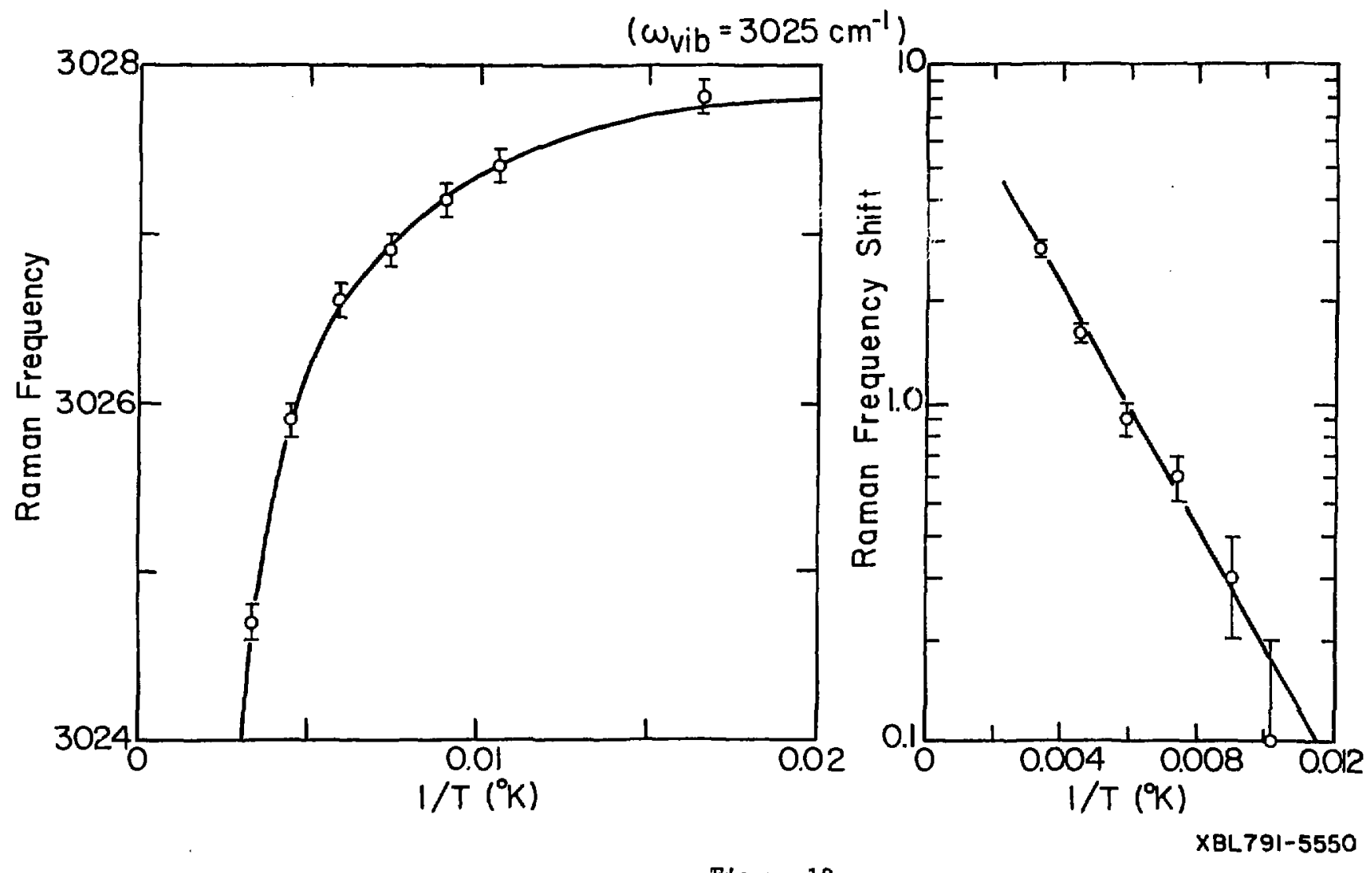

Figure 19 
This activation energy does Indeed match the energy of another molecular mode, namely, the methyl rock at $282 \mathrm{~cm}^{-1}$. Thus we would suggest that an excitation of the methyl rock at $282 \mathrm{~cm}^{-1}$ causes some change in the electron distribution in the ring, which in turn causes a shift of $-21.2 \mathrm{~cm}^{-1}$ in the frequency of the aromatic proton stretch.

\section{Low-Frequency Spectra and the Exciton Model}

In carrying out an exchange aralysis, it is obviously necessary to know the energies of the low-frequency modes of the molecule. In some cases, additional information can be obtained from a careful spectral study of this frequency region as a function of temperature and concentration.

The first example is shown in Figure 20 which shows the spectrum of a mixed crystal in the region of the $\mathrm{CH}_{3}$ and $\mathrm{CD}_{3}$ in plane methyl rocks. In $\mathrm{h}_{14}$-durene, there are two modes coupled to the methyl rock, and in $\mathrm{d}_{14}$-durene, there are three modes coupled to this motion. However, in each molecule two different values of $\tau$ are observed for this mode. This apparent contradiction ${ }^{22}$ is resolved by the data, since in both molecules the methyl rock transition is clearly split into two components at the lowest temperature. Previous work in $h_{14}$-durene 32,33 has treated the peaks around $281 \mathrm{~cm}^{-1}$ as one band. The possibility that the two peaks represent a Davydov splitting of a single transition can be ruled out, since the splitting is independent of isotopic dilution, and the higher-energy peak does not "borrow" intensity from the lower-energy one with increasing temperature. It is safe to conclude that Figure 20 illustrates two symmetry modes for the methyl rock, and that the two modes have different $\tau^{\prime} s$. This conclusion is summaried in Figure 21. 
Figure 20. Durene exhibits two Raman-active methyl rocking modes, which are resolved spectroscopically at low temperature, but coalesce by $90^{\circ} \mathrm{K}$. In addition, it should be noted that these transitions broaden and shift with increasing temperature, in agreement with the proposed vibrational exciton model. 
METHYL ROCK SPECTRA

IN $1: 3 \quad d_{14}: h_{14}$-DURENE

$d_{14}$-durene $130^{\circ} \mathrm{K}$

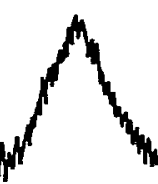

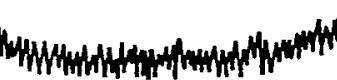

$$
h_{14} \text {-durene }
$$

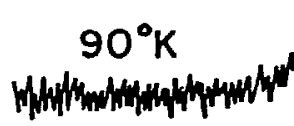

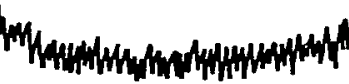
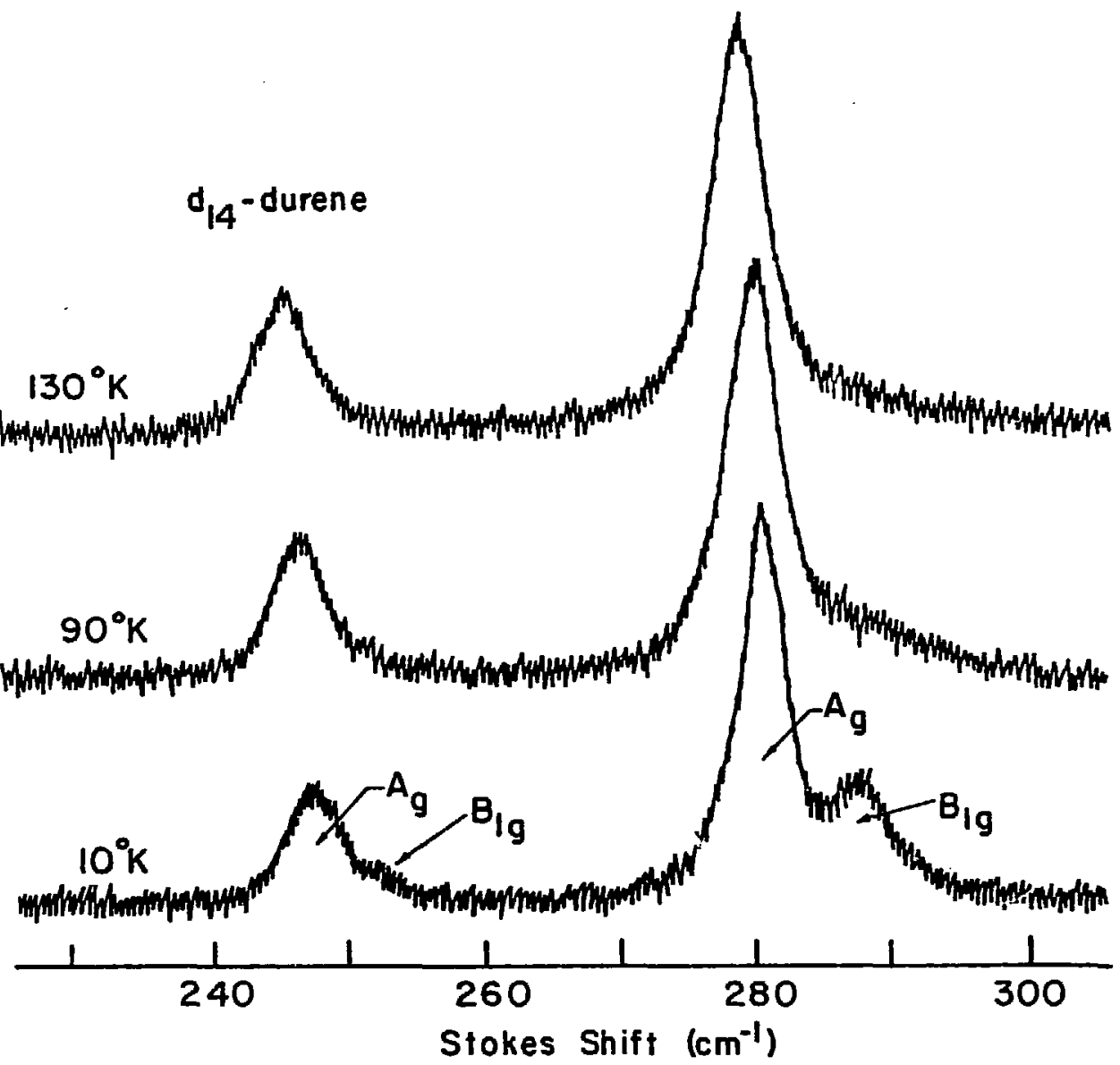

XBL 794-6108

Figure 20 
Figure 21. An illustration of the two methyl rocking motions observed In Figure 20. The different values of $T$ allow the assignment of the coupling scheme between the methyl rocks and the high-frequency modes. 
RAMAN ACTIVE IN-PLANE METHYL ROCKING MODES IN DURENE

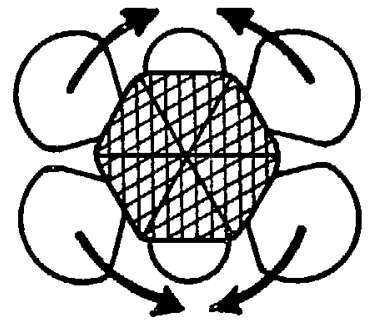

$A_{g}$ Symmetry

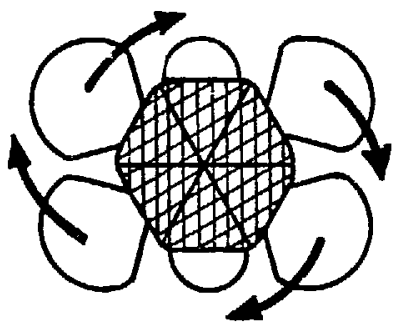

$B_{\text {Ig Symmetry }}$

Methyl Rock as Observed Dephasing Channel

\begin{tabular}{|c|c|c|c|c|}
\hline & $\begin{array}{l}\text { PEAK } \\
\left(\mathrm{cm}^{-1}\right) \\
\end{array}$ & $\begin{array}{l}E i \\
\left(\mathrm{~cm}^{-1}\right) \\
\end{array}$ & $\begin{array}{l}\tau \\
\text { (psec) }\end{array}$ & $\begin{array}{l}\text { Symmetry of } \\
\text { Methyl Rock }\end{array}$ \\
\hline \multirow{2}{*}{ Pure $h_{14}$-durene } & 2970.3 & $265 \pm 6$ & 0.19 & $A_{g}$ \\
\hline & 3027.5 & $263 \pm 19$ & 0.36 & $B_{1 g}$ \\
\hline \multirow{3}{*}{ Pure $d_{14}$-durene } & 2035.4 & $252 \pm 10$ & 0.66 & $B_{I g}$ \\
\hline & 2191.1 & $240 \pm 20$ & 0.30 & $\mathrm{Ag}$ \\
\hline & 2225.9 & $240 \pm 15$ & 0.36 & $A_{g}$ \\
\hline
\end{tabular}

XBL794-6105 
A more important question concerns the dynamics of the energy transfer in the low-frequency modes. On the basis of the observed Raman linewidths of these modes, their dephasing times can be calculated. These dephasing times are tabulated in column 6 of Table 4 and are, in several cases, longer than the measured value of $\tau$ by up to a factor of ten. ${ }^{22}$ This implies that some process which contributes to $\tau$ does not contribute to the linewidth; we postulate that this process is coherent, resonant energy transfer of low-frequency vibrational quanta. In a manner analogous to that of Reference 21 , we separate the two contributions to $\tau$ Into a resonant and a relaxation term:

$$
1 / \tau=1 / \tau_{\text {res }}+1 / \tau_{\text {rel }}
$$

Using the measured values for $\tau$ from the exchange analysis, and approximating $\tau_{r e l}$ by the dephasing time obtained from the appropriate lowfrequency mode linewidth, we can obtain an estimate for ${ }^{\tau}{ }^{-1}$, the resonant energy transfer rate. If this transfer rate exceeds the relaxation rate, a given excitation can traverse more than one molecule In its lifetime, and can properly be described as being partially delocalized or "excitonic" in character. Exciton models developed previously for electronic triplet state excitons in molecular crystals ${ }^{34-36}$ can be used as a context for the discussion and analysis of these "vibrational exc1tons." 37,38 We present here only a simple outline of a vibrational exciton model, in order to fllustrate the possibilities inherent in such a concept. It is not to be expected that complete understanding of vibrational excitonic properties will be realized here, but it will be demonstrated that a useful and interesting semiquantitative picture can be obtained from the data in a straightforward way. 
An examination of the low-frequency linewidths, in conjunction with the values of $T$ obtained from the exchange analysis, can yield an Indication of the degree of delocalization present in each low-frequency mode. We define a quantity $\langle n>$, the average number of coherent jumps for a given mode:

$$
\langle\mathbf{n}\rangle=\frac{\tau_{\text {rel }}}{\tau} \text {. }
$$

This quantity is tabulated in column 7 of Table 4, and it can be seen that the largest values of $\langle n\rangle$ occur for the modes at $2191.1 \mathrm{~cm}^{-1}$ and $2225.9 \mathrm{~cm}^{-1}$, which are both coupled to the $A_{g}$ methyl rock motion at $240 \mathrm{~cm}^{-1}$. In agreement with Reference 31, we advance the assumption that this methyl rock exhibits significant excitonic character, and present the results of an investigation of this hypothesis. This investigation has relied upon direct observation of the Raman spectrum of the methyl rock as a function of temperature and concentration, and also indirect observation through exchange analysis of the coupled highfrequency mocie at $2225.9 \mathrm{~cm}^{-1}$.

The concentration dependence of the low-temperature spectrum of the methyl rock in both $\mathrm{d}_{14}$-durene and $\mathrm{h}_{14}$-durene is given in Table 5 . The important point to note is that as each pure species is substituted with Its isotopic analog, the methyl rock peak shifts to lower frequency by approximately $3 \mathrm{~cm}^{-1}$. This can be explained readily within the framework of the vibrational exciton picture; In which the exciton $\vec{k}$ states are a band of linear combinations of isolated molecular states. In a pure crystal, the location of the spectroscopically allowed $(\vec{k}=0)$ transition within this band of states determines the observed peak frequency. The observed frequency in a dilute species, on the other hand, is simply the transition frequency of the isolated molecule, since resonant energy 
Table 5. Concentration Dependence of $A_{g}$ Methyl Rock Lineshapes $1 n h_{14}-$ and $d_{14}$-durene

\begin{tabular}{|c|c|c|c|c|}
\hline Environment & $100 \% \mathrm{~d}_{14}$-durene & $\begin{array}{l}50 \% \mathrm{~d}_{14} \text {-durene } \\
50 \% \mathrm{~h}_{14} \text {-durene }\end{array}$ & $\begin{array}{l}25 \% \mathrm{~d}_{14} \text {-durene } \\
75 \% \mathrm{~h}_{14} \text {-durene }\end{array}$ & $\begin{array}{l}10 \% \mathrm{~d}_{14} \text {-durene } \\
90 \% \mathrm{~h}_{14} \text {-durene }\end{array}$ \\
\hline \multicolumn{5}{|l|}{$d_{14}$-durene } \\
\hline $\begin{array}{l}\text { peak position } \\
\left(\mathrm{cm}^{-1}\right)\end{array}$ & 249.2 & 248.4 & 247.6 & 246.6 \\
\hline FWHM $\left(\mathrm{cm}^{-1}\right)$ & 4.0 & 5.2 & 5.0 & 4.0 \\
\hline \multicolumn{5}{|l|}{$h_{14}$-durene } \\
\hline peak position & & 278.4 & 280.4 & 280.8 \\
\hline FWHM $\left(\mathrm{cm}^{-1}\right)$ & & 4.4 & 3.6 & 3.6 \\
\hline
\end{tabular}


transfer is forbidden and the exciton picture is invalid. The data of Table 5 therefore implies that, in the pure crystal, the transition frequency of $\vec{k}=0$ is raised approximately $3 \mathrm{~cm}^{-1}$ above the transition frequency of the isolated molecule, and hence the full width of the exciton band is approximately $6 \mathrm{~cm}^{-1}$. It should be mentioned that the data cannot be accounted for by assuming that the frequency shift results from a change in intermolecular potential uron lsotoplc substitution, since both peaks shift in the same direction with dilution.

Further evidence for the validity of the exciton plcture emerges from a consideration of the temperature dependence of the linewifth of the $\mathrm{d}_{14}$-durene methyl rock, shown in Table 6 . It is known from studies of electronic exciton spectra ${ }^{34}$ that, at elevated temperatures, phonon scattering serves to localize the excitations. In effect, this results in the redistribution of oscillator strength into exciton states other than $\vec{k}=0$, and consequently as the temperature is increased, the linewidth broadens to reflect the entire range of $k$ states comprising the exciton band. 34,35 This explains the behavior observed in $\mathrm{d}_{14}$-durene, where the methyl rock broadens from $4.0 \mathrm{~cm}^{-1}$ at low temperature to 6.2 $\mathrm{cm}^{-1}$ at $275^{\circ} \mathrm{K}$, in agreement with our estimate of $6 \mathrm{~cm}^{-1}$ for the exciton bandwidth.

Although this evidence is consistently in support of the proposed exciton picture, a more crucial test of this hypothesis lies in its ability to explain the observed behavior of $\tau$ as a function of concentration. To model the concentration dependence, we assume that the relaxation time for decay of low-frequency modes into lattice modes ( $\tau_{r e l}$ in Equation 5) is independent of concentration. This assumption is equivalent to asserting that for lattice modes, the difference in frequency between a given $\mathrm{d}_{14}$-durene mode and the corresponding 
Table 6. Temperature Dependent Linewidth of $\mathrm{A}_{\mathrm{g}}$ Methyl Rock in $\mathrm{d}_{14}$-durene (FWHM in $\mathrm{cm}^{-1}$ )

\begin{tabular}{lllllll}
$10^{\circ} \mathrm{K}$ & $90^{\circ}$ & $130^{\circ}$ & $170^{\circ}$ & $225^{\circ}$ & $250^{\circ}$ & $275^{\circ}$ \\
\hline 4.0 & 4.6 & 4.8 & 6.0 & 6.1 & 6.1 & 6.2
\end{tabular}


$\mathrm{h}_{14}$-durene mode 1 s less than the linewidths of those modes, or in other words, the trap depth for lattice modes is less than their bandwidth (the amalgamation 11 init). 36,39 Conversely, the resonant energy transfer time ( $\tau_{\text {res }}$ in Equation (34) may be concentration dependent, since from Figure 20 the lineshapes of the methyl rock in the two isotopic species are well separated. If the molecule under observation is present in a concentiation $c$, its effective $\tau_{\text {res }}$ will be a function of the concentration and the pure crystal resonant transfer time, rres,pure:

$$
1 / \tau_{\text {res }}=\alpha(c) 1 / \tau_{\text {res, }} \text { pure }
$$

Substitution of (7) into Equation (5) now gives:

$$
\frac{1}{\tau}=\alpha(c)\left[\frac{1}{\tau_{\text {res, pure }}}\right]+\frac{1}{\tau_{\text {rel }}} \text {. }
$$

The function $\alpha(c)$ characterizes the type of energy transfer: in a localized, non-excitonic picture, where nearest neighbor interactions are important, $\alpha(c)$ decreases smoothly from $a(c)=1$ at $c=1$ to $\alpha(c)=0$ at $c=0$; in a delocalized, excitonic picture, where resonant interactions extend over several lattice sites, $\alpha(z)$ remains constant from $c=1$ down to some critical concentration (typically 10\%), below which Anderson localization takes place. 36 Thus, if $\tau$ increases with dilution, it Indicates non-excitonic behavior, while a constant $\tau$ is indicative of exciton behavior.

Examples of both were observed in our series of mixed crystal studies, and the data for one of each type is given in Table 7. In agreement with our expectations concerning the $A_{g}$ methyl rock, $\tau$ for the coupled high-frequency mode at $2225 \mathrm{~cm}^{-1}$ remains constant as a function of dilution. This behavior, taken with the other avallable evidence, 
Table 7. Concentration Dependence of Exchange Parameters of C-D Stretch Modes in $\mathrm{d}_{14}$-durene

$$
\underline{2225 \mathrm{~cm}^{-1} \text { Stretch }}
$$

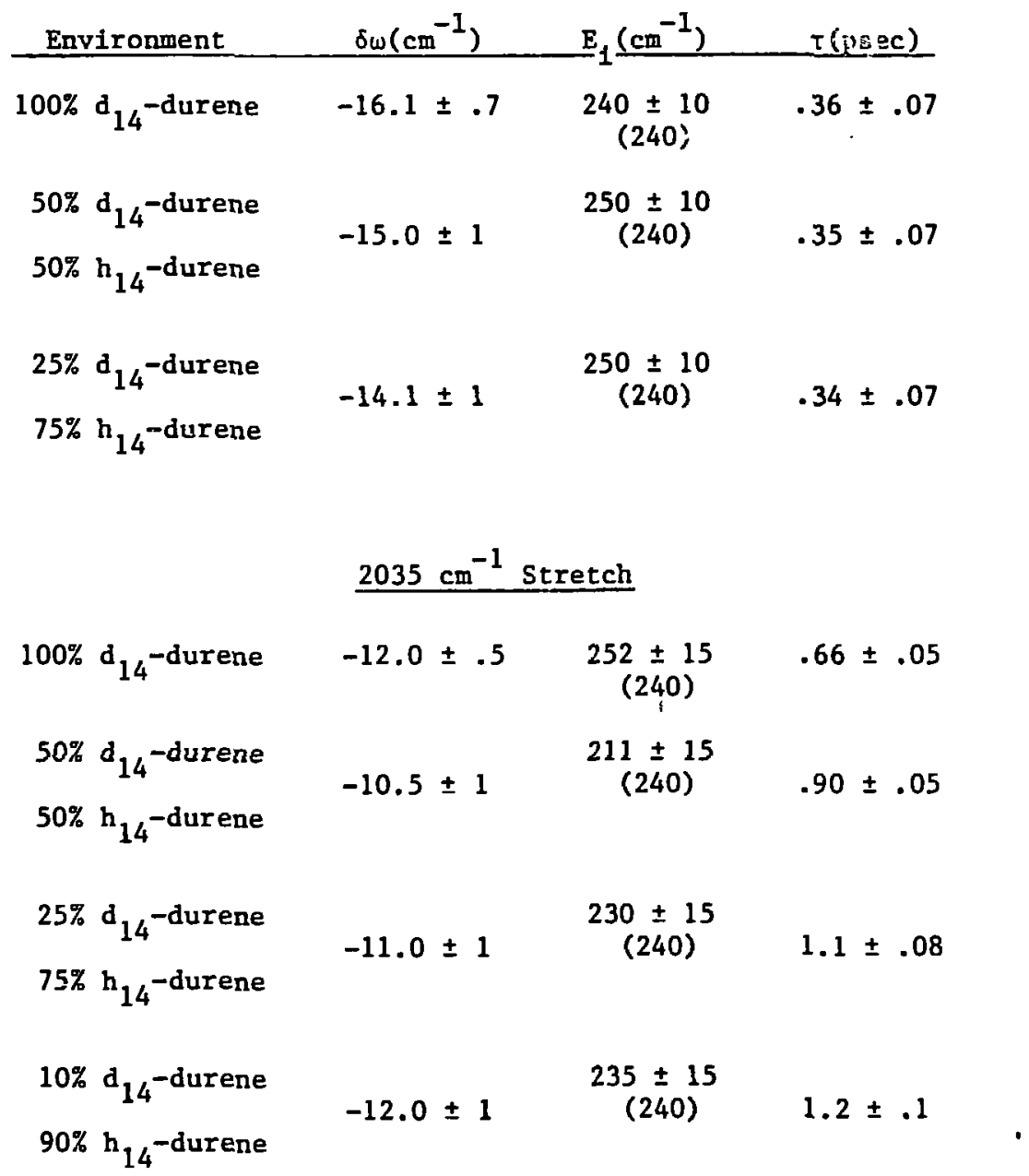


provides justification for the use of the exciton model in describing the dynamics of this mode.

A different trend appears in the data from the $2035 \mathrm{~cm}^{-1}$ mode, which is coupled to the $\mathrm{B}_{1 \mathrm{~g}}$ methyl rock. In this case, $\tau$ lengthens by a factor of two over the concentration range studied, as given in Table 7 and shown graphically in Figure 22. By a simple linear extrapolation of the data, we estimate $\tau_{\text {rel }}$, the relaxation time into lattice modes for the $\mathrm{B}_{1 \mathrm{~g}}$ methyl rock, to be $1.4 \pm .1 \mathrm{psec}$, and the energy transfer time, 'res', to be $1.2 \pm .1$ psec. The value of $\tau_{\text {rel }}$ obtained in this way differs by less than a factor of two from the inverse linewidth of this mode. Based on these values, we conclude that the exchange mechanism for this mode consists of excitations and de-excitations of isolated molecules.

The results of the foregoing analysis lend new insight into the detailed nature of the exchange process. They 1llustrate how spectral evidence of various kinds may be used to characterize the exchange mechanism in terms of 1 ts localized or delocalized nature, and how the linewidth of a low frequency mode, together witl: exchange analysis of the coupled high-frequency modes, can be used to provide a direct measure of the degree of excitonic character present in that low-frequency mode. The data suggests that, in this molecule, different degrees of delocalization occur for different low-frequency modes, but that the concept of a delocalized vibrational exciton must not be neglected in modeling the dynamical behavior of vibrations in molecular solids.

\section{Conclusion}

The results presented here provide strong evidence for the utility of the simple exchange model. The presence of intermediate exchange and the excellent correspondence between calculated activation energies and 
Figure 22. The data is for the $2035 \mathrm{~cm}^{-1}$ mode, which is coupled to the $B_{1 g}$ methyl rock. The slope of the line gives an estimate for $\tau_{\text {res }}$ of $1.2 \pm .1$ psec; the intercept gives $\tau_{\text {rel }}$ as $1.4 \pm .1$ psec. These values suggest that the $B_{1 g}$ methyl rock does not exhibit signiflcant excitonic properties. 
DEPENDENCE OF INVERSE EXCHANGE TIME ON CONCENTRATION ( $2035 \mathrm{~cm}^{-1}$ C-D STRETCH $d_{14}$-DURENE)

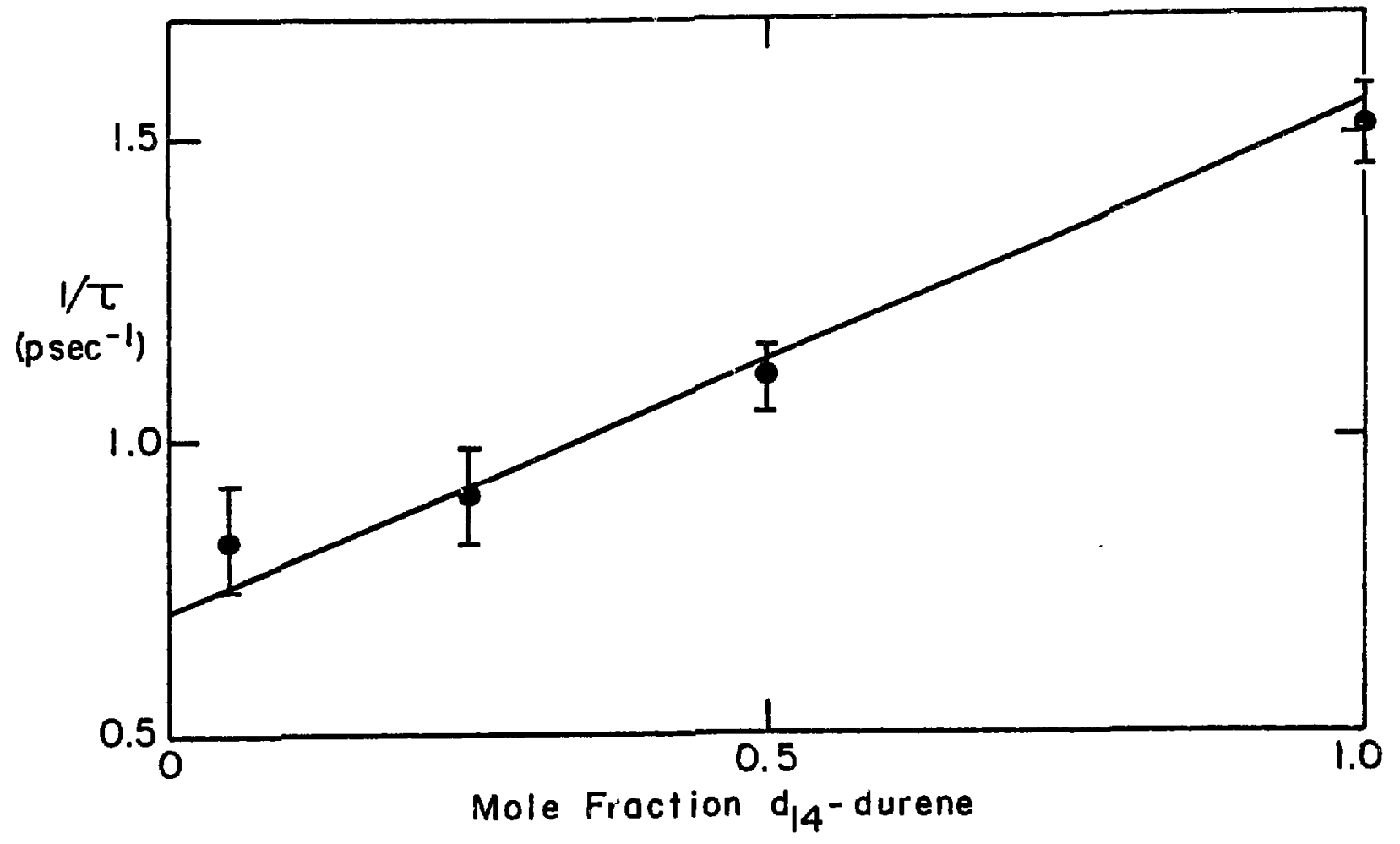

Figure 22

XBL794-6106 
known energies of low-frequency modes constitute 1mportant support for the exchange model. Of particular interest is the successful observation by infrared spectroscopy of the combination bands predicted by exchange theory. This latter result, along with qualitative observations of the temperature dependent frequency shifts in these molecules, tends to corroborate exchange theory in preference to other proposed models.

The results of the exchange theory analysis applied to highfrequency modes, plus measurements of the linewidths of the coupled low-frequency modes, have led to the conclusion that the low frequency methyl group modes of these molecules exhibit significant delocalized or "excitonic" character. The assumption of exciton behavior not only explains the observed low-frequency mode linewldths, but also enables a serles of mixed crystal studies to yleld insight into the relative importance of resonant and non-resonant energy transfer processes in the exchange mechanism.

The use of exchange theory to interpret this simple series of experiments has resulted in a detalled understanding of the dynamics of this system. Further applications of this theory to other such systems and, in particular, to the problem of vibrational deptasing in liquids is currently in progress. 11 
CHAPTER IV

\section{PICOSECOND SPECTROSCOPY}

While the results of the previous three chapters represent a means by which detailed dynamical Information can be obtained, the method of Raman spectroscopy suffers from two inherent shortcomings: the observed lineshape is a composite of dynamic and static effects, the latter giving rise to inhomogeneous broadening; and the processes of population relaxation and dephasing both contribute to the lineshape and cannot be separated. Although exchange theory will remain applicable as long as the contributions from inhomogeneous broadening and population relaxation remain small or are independent of temperature, it is nevertheless desirable to have a method for measuring $T_{1}$ and $T_{2}$ separately. Raman scattering of picosecond pulses offers a means by which this can be accomplished, provided that the vibrational mode of interest has stimulated Raman gain. In this type of experiment, an intense, coherent picosecond pulse prepares the sample in a well-defined state of excitation. This state decays and is subsequentiy probed by a weaker pulse, whose scattering characterizes the amount of excitation remaining in the ensemble. Owing to the coherent nature of the excitation, wave-vector matching conditions apply to this probing process. By choosing the appropriate experimental geometry, the probe scattering can be either coherent or incoherent, thereby measuring either $T_{2}$ ot $T_{1}$. In the case where the coherent decay is observed, the wave-vector matching conditions can be used to restrict the probe scattering to a selectively chosen subgroup of the inhomogeneous distribution, thus determining the homogeneous dephasing time, $T_{2 *^{*}}$ These capabilities make this technique a 
compliment to the type of spontaneous Raman studies described in the first three chapters.

Both the experimental apparatus 8,12 and the theory of transient stimulated Raman scattering ${ }^{6,9}, 12,13$ have been described \&n detail elsewhere, and will only be summarized here along with some early unpublished results. This chapter will focus on the methods used for fitting and understanding experimental data, and some of the conclusions based on these calculations will be examined.

1. Summary of Design and Execution of Picosecond Scattering Experiments

The laser used in these experiments and its operation has been discussed at great length, ${ }^{1-9,12}$ expecially in Reference 12 . Figure 23 illustrates the apparatus schematically and explains briefly its operation. A pulse train is generated by a passively mode-locked Nd:glass oscillator, anä a spark gap and Pockels cell is used to select a single pulse from the early part of this train (see Fig. 24). This pulse passes through three stages of amplification, attaining an energy of 50-100 mJ. It is then passed through a saturable absorber to compress the wings of the pulse, and to attenuate that fraction of the barkground pulse trair. ch was not rejected by the pulse selector. A : : 1 of KDP provides second harmonic generation, resulting in a $5300 \mathrm{~A}$ pulse of approximately $10 \mathrm{~mJ}$ and 6-8 picoseconds FWHM. The spectral width of the pulses is generally within a factor of 2 of the uncertainty principle jimit, although there is some shot-to-shot variation in the pulse spectrum.

The experimental set up is tllustrated in Figure 25 . The pulse emerging from the doubling crystal is split into two parts, an exciting and a probing pulse. The latter is passed through an optical delay line 
Figure 23. Diagram of Nd:glass laser. The laser oscillator is formed by mirrors $M I$ and $M 2$, the latter being partly transmitting. A contacted dye cell $D$ holds the flowing saturable dye which provides mode locking. The cavity also includes the Brewster-angled Nd:glass rod $L$, and an aperture $A$ which selects for $\mathrm{TEM}_{00}$-mode. The pulse train emerging from $\mathrm{M} 2$ passes through a half-wave rotator $\lambda / 2$ and two crossed Glan-Thompson polarizers GP. A portion of the pulse train is reflected by the beam splicter BS into a spark gap SG; when a 1ight pulse of sufficlent intensity enters the spark gap, it breaks down and releases a high-voltage pulse to the Pockels cell PC. The polarization of the next pulse in the laser pulse train is rotated, causing it to pass through the second GP undeflected. It is then passed twice through amplifier $A_{1}$ and once through $A_{2}$, and finally through a KDP doubling crystal and on to the experiment. 
MODE LOCKED Nd-GLASS LASER SYSTEM

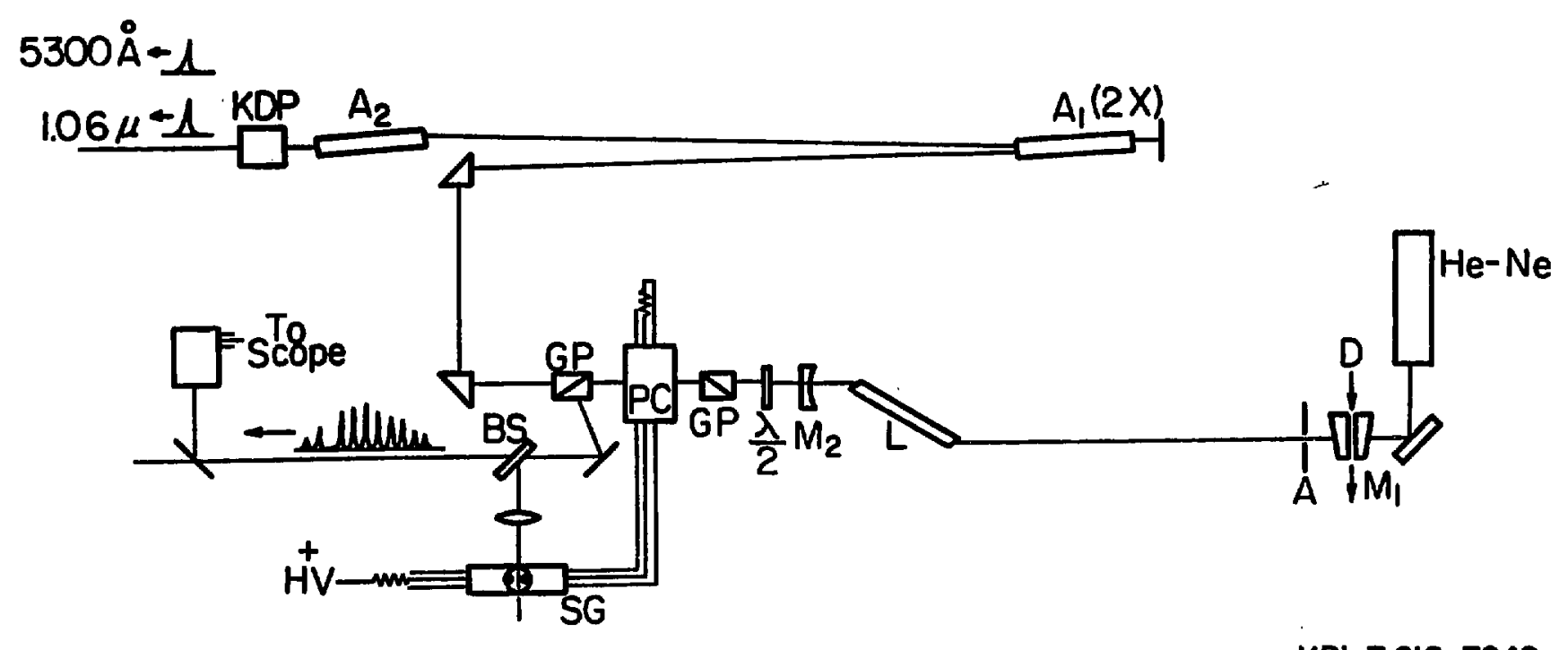

XBL 7612-7942

FIgure 23 
Figure 24. These are five typical pulse trains created by the laser system of Figure 23. In $A$, all three trains are cleanly mode-locked and are sitible for doing an experiment. In $B$, the traces show the presence of extraneous pulses. Such shots must be rejected in an experimental situation. The bottom trace in $B$ shows a missing pulse, which has been selected by the Pockels cell and sent through the amplifiers to the experiment. For best pulse quality, pulse selection should occur as shown, just before the peak of the pulse train envelope. 


\section{EXAMPLES OF MODE-LOCKED PICOSECOND PULSE TRAINS}

(A)

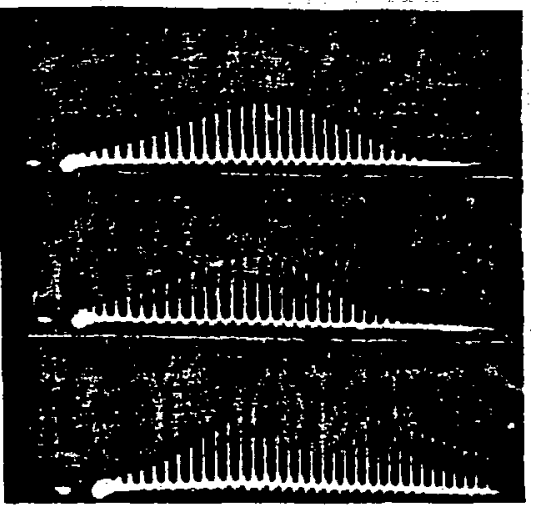

(B)

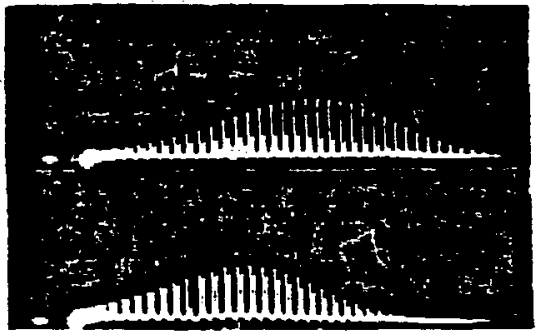

Figure 24 
Figure 25. This figure describes the setup for performing a $T_{2}$ measurement. A $\mathrm{T}_{1}$ measurement would use the 1.06 fundamental pulse for the exciting pulse, but the setup is otherwise similar to what is shown here. A beam splitter BS splits the pulse into two parts: the exciting beam is focussed by telescope $T_{2}$ into the cell. The probe beam passes through an optical delay line DL, is filtered by $F_{1}$ to remove unwanted background light (generated by nonlinear processes in the KDP doubling crystal), is passed through a $\lambda / 2$ plate to rotate its polarization, and is focussed into the cell by telescope $T_{1}$. Photomultiplier tube PMT2 and filter $F_{3}$ detect the Stokes radiation generated by the exciting pulse. An aperture $A$, filter $F_{2}$, and the spectrometer SP allow the photomultiplier tube PMT3 to read the probe scattered antiStokes radiation. In some applications, PMT3 is replaced by an optical multichannel analyzer. 
MEASUREMENT OF VIBRATIONAL DEPHASING TIME $\left(T_{2}\right)$ USING COHERENT ANTI-STOKES SCATTERING

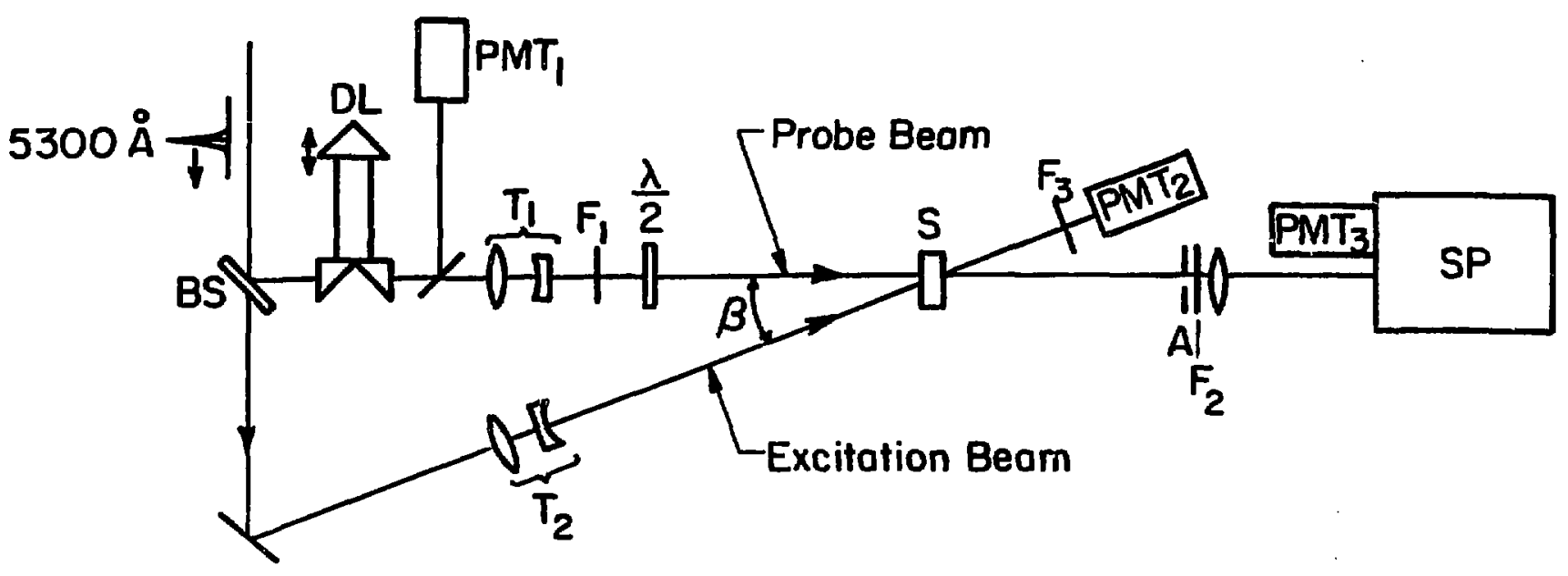

Ref: von der Linde, Laubereou and Koiser, Phys. Rev. Lett., 26, 954 (1971)

Figure 25 
which allows the temporal overlap of the pulses to be adjusted, while the beams axe crossed spatially inslde the sample cell at a position just before the exit window. Photomultipliers monitor the incoming intensity of the probe beam and the scattered radiation from the excitation and probing processes. A half-wave plate inserted into the probe beam causes the pulses tn have orthogonal polarizations, which facilitates the discrimination of the weak probe scattering from unwanted background generated by the excltation pulse.

The experimental method is described by Figure 26, and discussed in more detail in the next section. The exciting pulse pumps population into the $v=1$ vibrationdl state by stimulated Raman scattering, in which a laser photon of wavevector $\vec{k}_{L}$ is annihtlated and a stokes photon $\vec{k}_{S}$ and an excited vibration $\vec{k}_{\text {vib }}$ are created:

$$
\vec{k}_{L}=\vec{k}_{S}+\vec{k}_{\text {vib }}
$$

Since the high-frequency vibrational modes typically studied in these experiments are nearly dispersionless, $\vec{k}_{v i b}$ may be of any length necessary to satisfy (37) for a given cholce of $\vec{k}_{s}$. In other words, $\vec{k}_{s}$ may point In any direction and the relationship (37) wil.1 be automatically satisfied. Since backward stimulated Raman scattering 13,60 is negligible In ultrashort pulses, the Stokes radiation is emitted in a cone around the direction of $\vec{k}_{L}$.

The probing pulse interacts with the excited population to produce anti-Stokes scattering, in which $\vec{k}_{v i b}$ and a photon $\vec{k}_{p}$ from the probe pulse are annihilated to produce an anti-stokes photon $\vec{k}_{A S}$ :

$$
\vec{k}_{P}+\vec{k}_{v i b}=\vec{k}_{A S} .
$$


Figure 26. (a) An energy level diagram for the exciting and probing processes. In the excitation step, a laser photon $w_{L}$ creates a virtual state; emission from this virtual state of a Stokes photon $\omega_{s}$ leaves an excited vibration. In the probing process, a probe pulse photon $\omega_{p}$ is absorbed by the $v=1$ population to produce a virtual state, which can then emit an anti-stokes photon $\omega_{\text {as }}$ to de-excite tile vibration. (b) Indicates the wavevector geometry governing the overall excitation and probing event, as discussed at length in the text. 
(a) STIMULATED RAMAN SCATTERING; EXCITATION AND PROBING PROCESSES

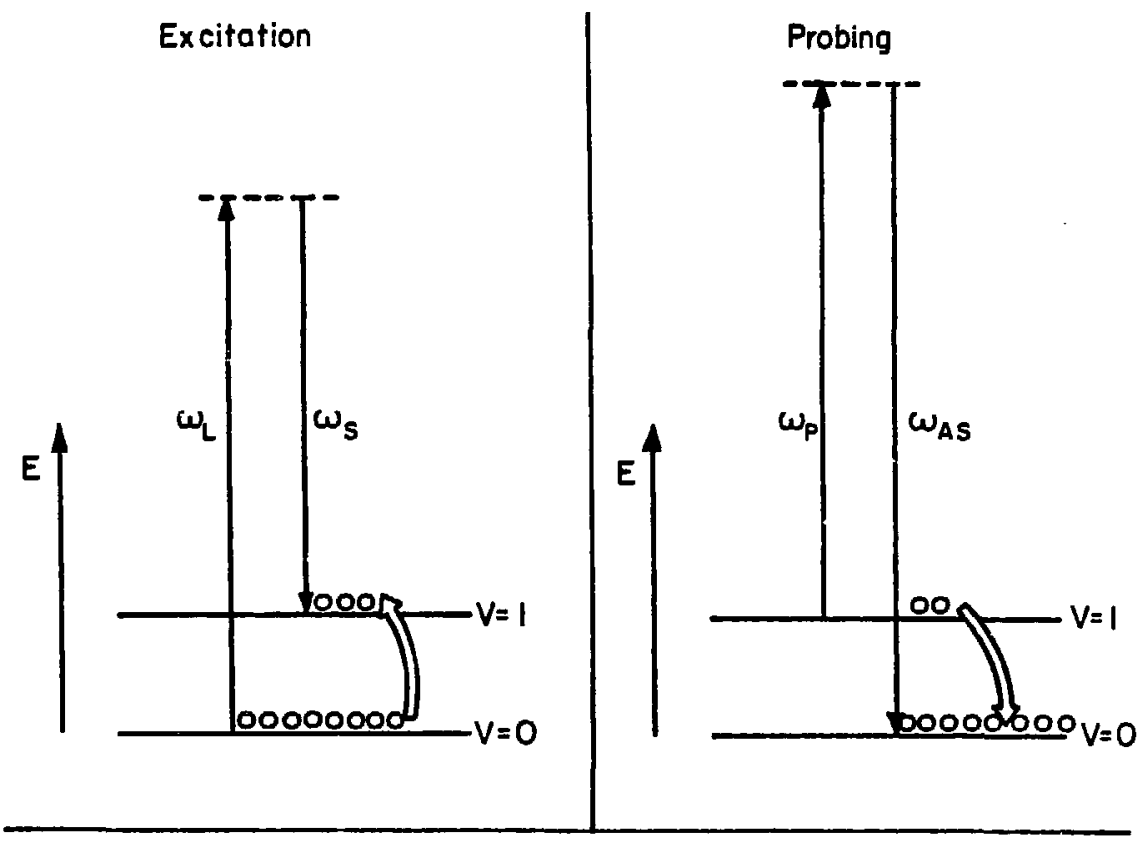

(b) WAVE VECTOR MATCHING GEOMETRY FOR COHERENT ANTI-STOKES SCATTERING

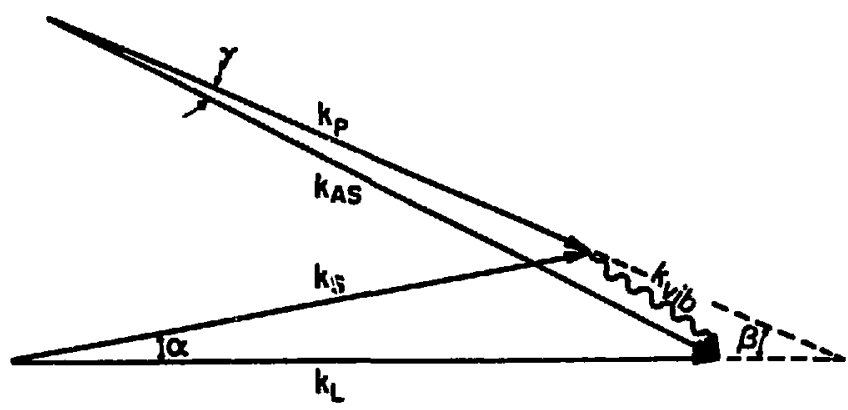

XBL 7612-7944

F1gure 26 
Wavevector matching for the overall exciting plus probing process is achieved only when

$$
\vec{k}_{\mathrm{L}}+\vec{k}_{\mathrm{P}}=\vec{k}_{\mathrm{AS}}+\vec{k}_{\mathrm{S}}
$$

which is obtained by adding (37) and (38). In a dispersionless medium, Equation (39) would be satisfied when $\vec{k}_{L}=\vec{k}_{P}$, $1 . e$, when the exciting and probing pulses are collinear and of equal frequency. Since the wavevectors depend on the index of refraction through the relationship $\vec{k}=n / \lambda$, in the presence of dispersion Equation (39) can only be satisfled when $\vec{k}_{L}$ and $\vec{k}_{P}$ are noncollinear by an angle $\beta$ (see Figure 26b). The consequences of this wavevector matching condition are what give this type of experiment its versatility, since by varying the probing interaction the experimenter can vary the information contained in the probe scattering signal. The wavevector mismatch, $\Delta \vec{k}$, is defined as:

$$
\Delta \vec{k}=\vec{k}_{\mathrm{AS}}+\vec{k}_{\mathrm{S}}-\vec{k}_{\mathrm{L}}-\vec{k}_{\mathrm{P}}
$$

The intensicy of the coherently scattered probe signal depends on $\overrightarrow{\Delta k}$ as 9,12

$$
I_{\operatorname{coh}} \alpha \frac{1}{1+2|\Delta \vec{k}|^{2} L^{2}}
$$

where the stimulated gain of the medium is described by $L$, the characterIstic length required for the stimulated Stokes excitation field to grow by one factor of $e$. When $|\Delta \vec{k}| \cong 0$, phase matching is achieved in the probing process, and the anti-Stokes signal propogates coherently through the sample. In this case, the observed anti-Stokes signal represents the overlap of the probing pulse shape with the coherent ensemble left behind by the exciting pulse; varylng the time delay between the two pulses 
allows the coherent decay time $\left(T_{2}\right)$ of the vibrators to be monitored, When $|\overrightarrow{\Delta k}|$ is large, on the other hand, $I_{\text {coh }} \rightarrow 0$ and only spontaneous anti-Stokes scattering is possible. The resulting incoherent signal, measured as a function of time delay, monitors the decay of the population $\left(T_{1}\right)$ of the exclted state. By performing these two experiments on the same vibration, one can determine both $T_{1}$ and $T_{2}$, which is not possible with spontaneous Raman scattering.

A second consequence of (40) appears when the vibrational ensemble is inhomogeneously broadened. The Inhomogeneous distribution can be degcribed by asgigning different resonance frequencies to different oscillators in the ensemble. Each individual osclllator is assumed to have the same Lorentzian spectrum, so the spectrum of the whole ensemble is a convolution of that Lorentzian with the Inhomogeneous distribution. The stimulated Raman emission from such an ensemble can be described theoretically, $3,6,9,10,12$ but applying the theory requires laborious numerical calculations. An effort to reduce the calculational complex1ty through an approximation ${ }^{6}$ appears quantitatively inadequate, ${ }^{10}$ although some important qualitative features are correctly described. The important point is that during the excitation process, the vibrations are driven at $w_{S}$, the frequency of maximum Stokes gain. Following the excitation, the relaxing vibrators oscillate at their individual resonance frequencies. 6 This means that the excitation process occurs predominantly at a single frequency, whereas the probing process occurs over a range of frequencles encompassing the whole Inhomogeneous IIneshape. The probe scattering can be calculated in the following way: the Stokes frequency $w_{S}$, the exciting, probing: and Stokes wavevectors $\vec{k}_{L}$ $\vec{k}_{P}$, and $\vec{k}_{S}$, the angle 6 between the exciting and probing beams, and the 
dispersion of the medium are fixed. Based on these parameters, the Stokes angle $\alpha$ can be calculated as $f \tau^{-1}$.ows for a given choice of antiStokes frequency and wavevector $\omega_{\mathrm{AS}}$ and $\vec{k}_{\mathrm{AS}}$ :

$$
\cos a=\frac{x z+\sqrt{(x z)^{2}+\left(y^{2}+x^{2}\right)\left(y^{2}-z^{2}\right)}}{x^{2}+y^{2}}
$$

where

$$
\begin{aligned}
& z=\left|\vec{k}_{A S}\right|^{2}-\left|\vec{k}_{L}\right|^{2}-\left|\vec{k}_{\mathrm{P}}\right|^{2}-\left|\vec{k}_{\mathrm{S}}\right|^{2}-2\left|\vec{k}_{\mathrm{L}}\right|\left|\vec{k}_{\mathrm{P}}\right| \cos \beta \\
& \mathrm{x}=-2\left(\vec{k}_{\mathrm{P}} \vec{k}_{\mathrm{S}} \cos \beta+\vec{k}_{\mathrm{L}} \vec{k}_{\mathrm{S}}\right) \\
& \mathrm{y}=2\left(\vec{k}_{\mathrm{P}} \vec{k}_{\mathrm{S}} \sin \beta\right) .
\end{aligned}
$$

Once $\alpha$ has been determined, the ant1-Stokes scattering angle $\gamma$ can be calculated by elementary geometry (see Fig. 26b). The important point of this calculation is that as the anti-Stokes frequency is varied across the Inhomogeneous lineshape, the scattering angle $\gamma$ will change This is 1llustrated in Figure 27, which is a calculation performed by the computer program KMATCH using Equations (42)-(45). Since $\gamma$ varies as a function of frequency, the scattering from different isochromats can be spatially resolved. By placing an aperture in the anti-Stokes beam, it is possible to selectively probe only a small part of the inhomogeneous distribution. The difference between selective and non-selective probing methods is illustrated in Figure 28. Using the selective probing method, a $\mathrm{T}_{2}$ time approaching the dephasing time of a single isochromat can be measured. Comparison of th:s time with the corresponding spontaneous Raman linewidth determines the presence or absence of inhomegeneous broadening. 
Flgure 27. An illustration of the wavevector matching calculation described by the text. The calculation is for the $2835 \mathrm{~cm}^{-1}$ line in methanol, and the angle $\beta$ is chosen to minimtze $\gamma$ at the center of the line. The graph shows how $\gamma$ varies with frequency, and can be used to determine how much of the inhomogeneous ensemble is being probed when an aperture is placed in the anti-stokes beam. 
FREQUENCY DEPENDENCE OF ANTI-STOKES ANGLE

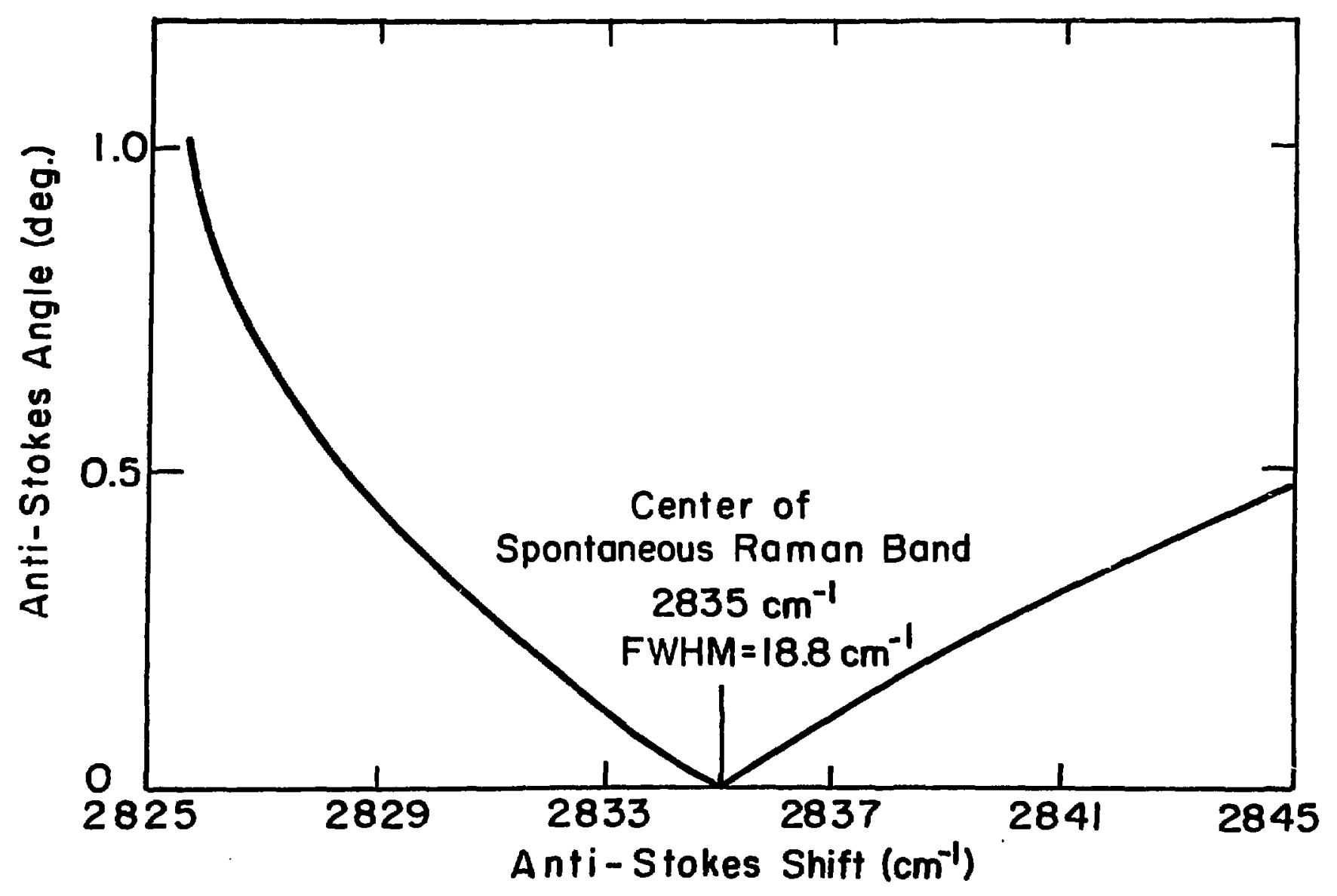


F1gure 28. "Selective" refers to the case of selective k-vector matching, where only a single component of the inhomogeneous ensemble generates the probe slgnal. "Non-selective" refers to the case where all components contribute to the observed scattering. A comparison of these two curves, or a comparison between the "selective" curve and the spontaneous Raman linewidth, allows the estimation of the importance of inhomogeneous broadening in the mode being observed. 
COHERENT PROBE SCATTERING FOR INHOMOGENEOUS VIBRATIONAL LINES

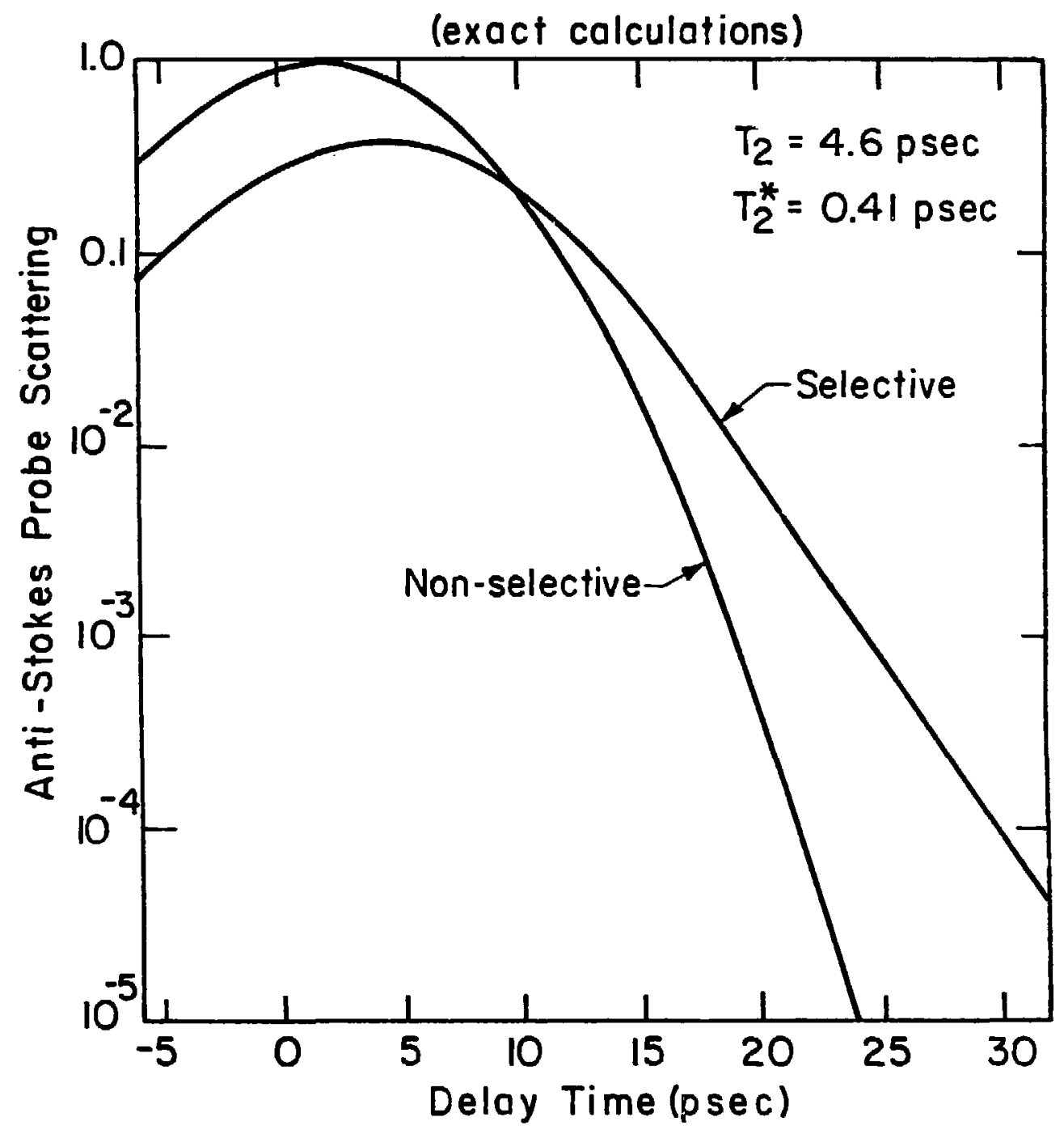

$X B L 783-4731$

Figure 23 
Despite the possibilities of this method, the practical difficulties Involved have thus far 1imited the value of this line of investigation. The measurement of homogeneous dephasing times by restricted wavevector matching is experimentally feasible only when the dispersion of the medium is large enough to cause $\gamma$ to vary rapidly as a function of $\omega_{A S}$. Nevertheless, even in the case where unrestricted wavevector matching is employed - in other words, no aperture is placed in the anti-Stokes beam some selectivity is still attained because of the stimulated nature of the excitation process. Figure 29 shows that ${ }^{10}$ individual oscillators within the Inhomogeneous ensemble are excited with unequal amplitudes, depending on their distance from the line center. An oscillator close to the center of the line receives relatively more excitation than those on the wings of the profile by orders of magnitude; because of this, the probe scattering signal that is ultimately measured in this experiment is weighted in favor of those isochromats near the center. Unfortunately, the exact amount of selectivity achieved in this way is difficult to estimats. In specific cases.

In spite of these theoretical reservations, we have performed several experiments using this unrestricted probing technique. Figures 30 and 31 show our data for liquid para-dimethyl benzene (xylene) and solid durene at room temperature. In addition, we have measured dephasing times for liquid durene at $353^{\circ} \mathrm{K}\left(\mathrm{T}_{2}=0.5 \mathrm{psec}\right)$, ethanol at room temperature $\left(T_{2}=0.5 \mathrm{psec}\right)$, and $1,1,1-$ trichloroethane at room temperature $\left(T_{2}=\right.$ $3.5 \mathrm{psec}$ ). In each case, the vibrational mode studied was a symmetric C-H stretch; the results in ethanol and trichloroethane were repeats of measurements originally made by the Kaiser group. $1,3,7-9$

These results are pr. sented here only for the purpose of 1llustrating the possibilities of this method, since other workers in this group have 
Figure 29. The relative excitation of the components of an inhomogeneous line. Within fifteen wavenumbers of the peak, the degree of excitation of an individual oscillator has dropped by over two orders of magnitude, indicating that the stimulated excitation process selectively excites components that are near the center of the inhomogeneous distribution. 
RELATIVE VIBRATIONAL EXCITATION DURING SRS FOR DIFFERENT HOMOGENEOUS COMPONENTS OF INHOMOGENEOUS VIBRATIONAL LINE

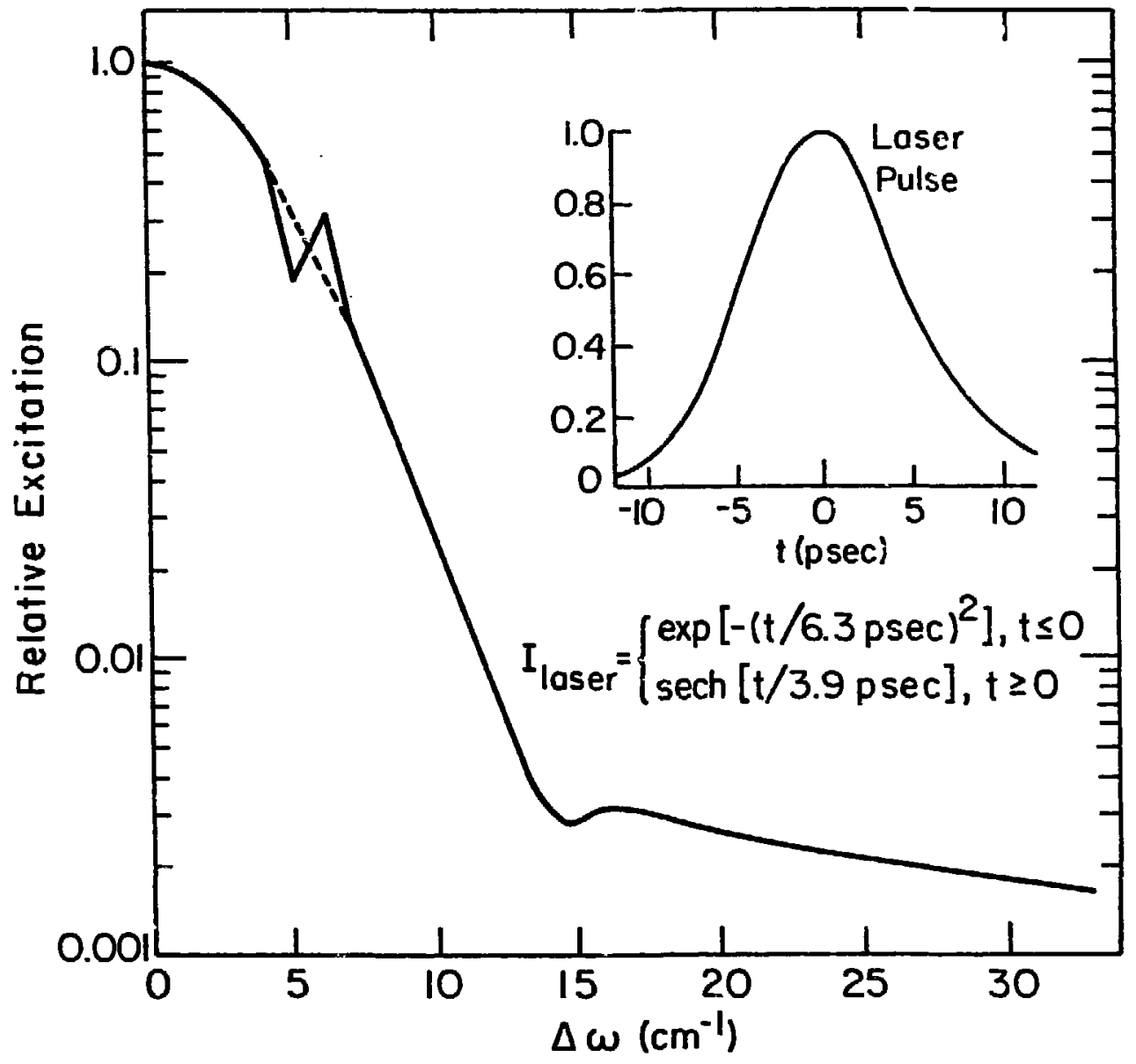

XBL 783-4736

Figure 29 
Figure 30. Coherent anti-Stokes scattering as a function of delay for the $2912 \mathrm{~cm}^{-1}$ vibration of $p$-xylene. The value of $\mathrm{T}_{2}$ determined by the fitting process described in the next section is 2.2 psec.

Figure 31. Coherent anti-Stokes scattering for the $2914 \mathrm{~cm}^{-1}$ vibration of solid durene. The fitting process gives the best $\mathrm{T}_{2}$ as $0.75 \mathrm{psec}$. 
COHERENT ANTI-STOKES SCATTERING IN $p$-XYLENE

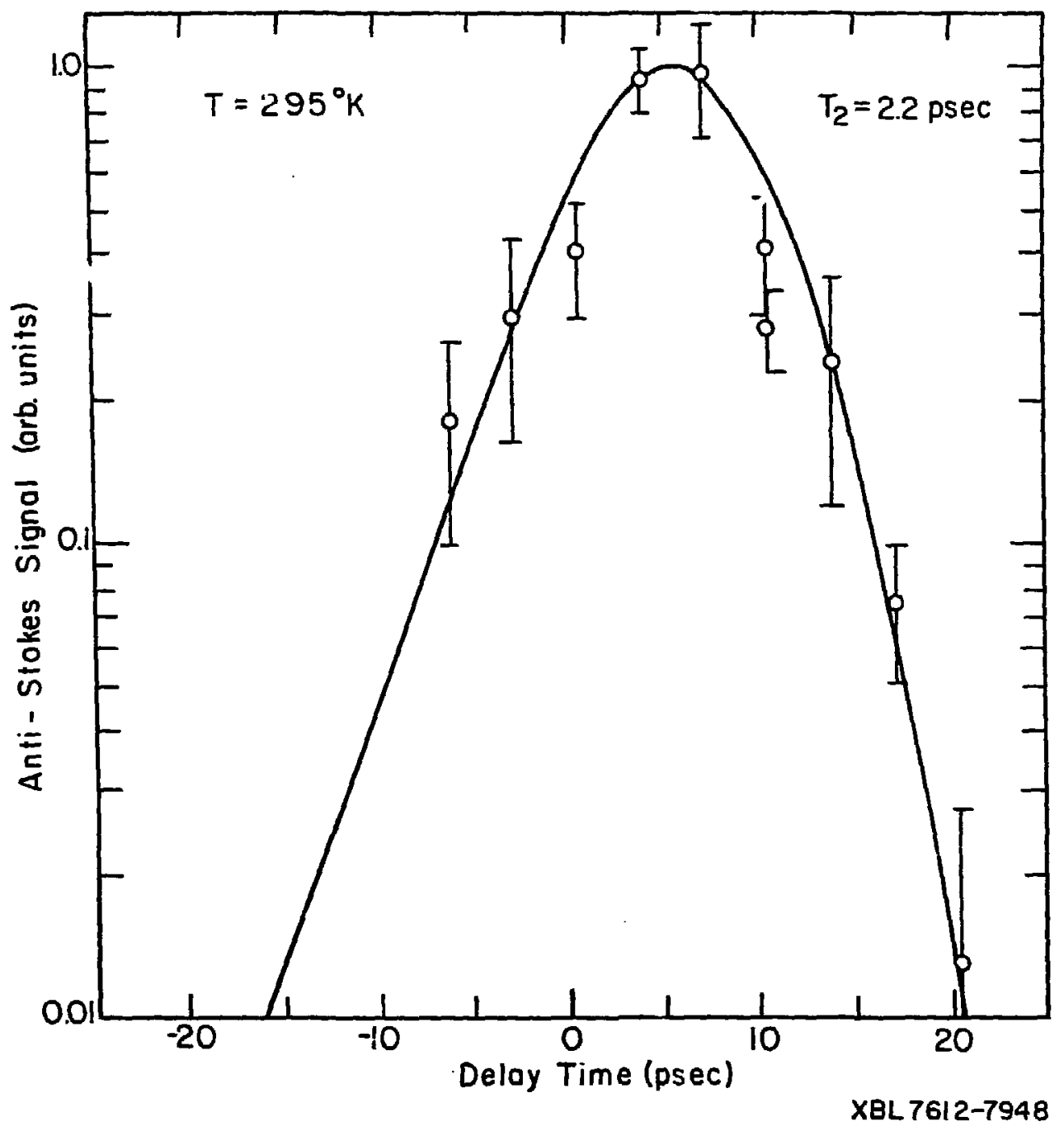

Figure 30 
COHERENT ANTI-STOKES SCATTERING IN DURENE (SOLID)

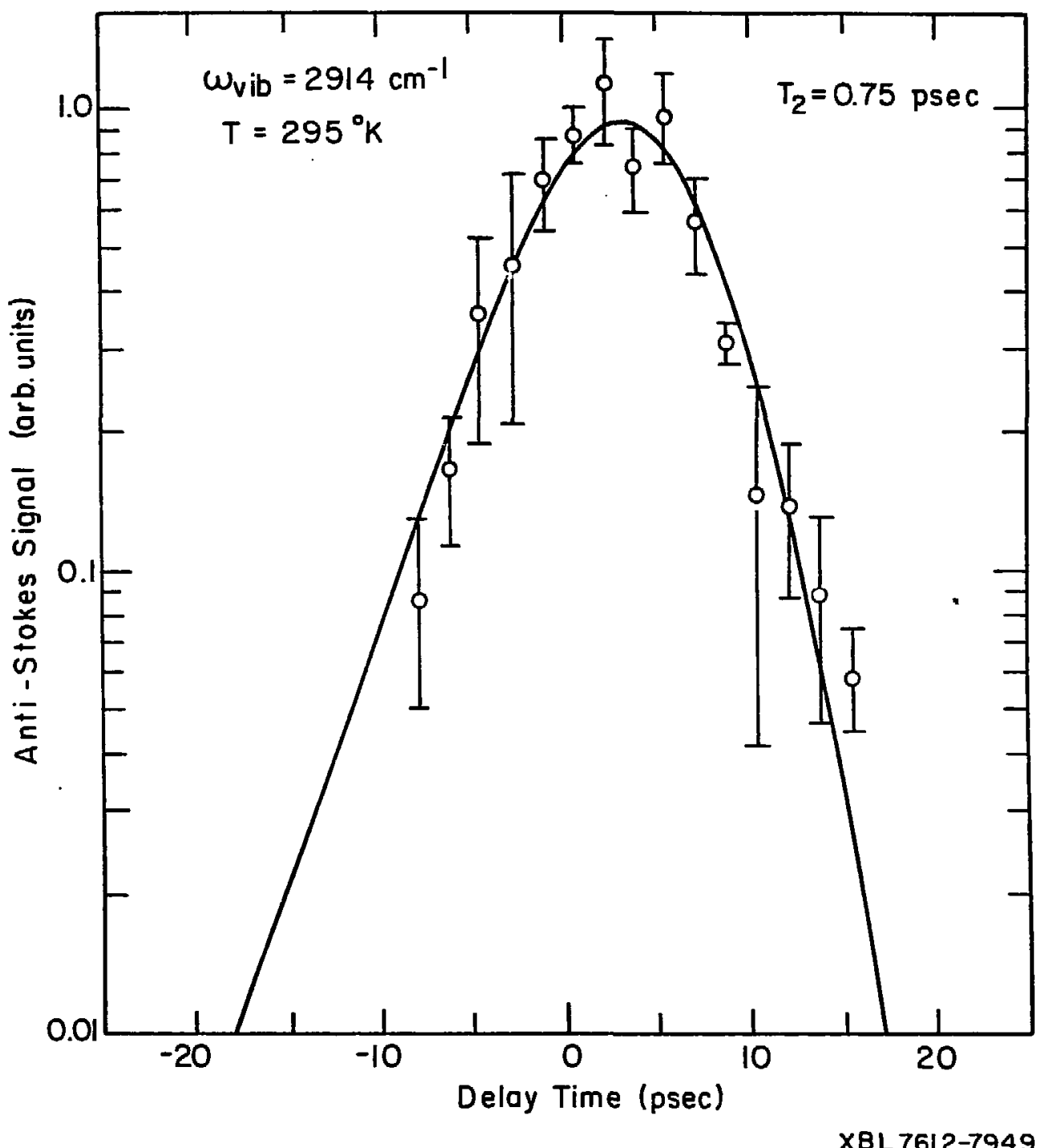

Figure 31 
recently deronstrated ${ }^{11}$ a superior method of measuring homogeneous dephasing times using a different experimental geometry. In these experiments, the exciting and probing beam traverse the sample collinearly, and the Stokes scattered probe signal is detected. 7,9 The requirements of restrictive wavevector matching are automatically satisfied, enabling a more reliable interpretation of the experimental curves. For a discussion of the results of these experiments, see Reference 11.

For measuring $T_{1}$ by incoherent anti-Stokes probing, the chief difficulty is the detection of the relatively small numbe: of spontaneously emitted photons. In contrast to the $T_{2}$ experiments, the interpretation of the curves is relatively straightforward since $T_{1}$ times are longer than $T_{2} \cdot{ }^{4,5}$ We have performed one such measurement, in $1,1,1$-trichloroethane $\left(T_{1}=6.6 \mathrm{psec}\right)$; this was a repeat of an experiment originally reported in Reference 4. An attempt was made to measure $T_{1}$ in solid durene, but did not meet with success and was finally abandoned. To digress a moment, one possible explanation for the failure of this experiment is the recent observation of the importance of self-phase modulation In the stimulated Raman excitation process, particularly for moderately oized molecules such as durene and xylene. As will be discussed in detail in Chapter $v$, the stimulated Stokes excitation spectra of these molecules show enormous broadening and a lack of shot-to-shot reproducibility. The unreliable nature of the excitation process could not only account for the problems encountered attempting to measure $T_{1}$ in durene, but also calls into question the validity of the $T_{2}$ times measured in xylene and in both liquid and solid durene.

For small molecules which do not have the problem of phase-modulation, these picosecond techniques represent a unique source of experimental information. Despite the obstacles, these methods should prove to be useful 
tools in the future study of vibrational dynamics.

\section{Calculation of Anti-Stokes Scattering Curves}

A theoretical descr:-tion of transient Stimulated Raman scattering was first carried out by Carman, ${ }^{13}$ and other workers subsequently developed this theory. to include the anti-Stokes probing processes described in the previous section. 3,6-10,12 Numerical calculations based on this theory can be used to show how the experimentally observed probe signal varies with delay time between the pulses. The parameters which enter the calculation include the exciting and probe pulse shapes, the intensities of the pulses, the steady-state stimulated Raman gain factor, and the dephasing time (or relaxation time for a $\mathrm{T}_{1}$ experiment) of the vibration. If the gain factor and the properties of the laser pulse are known, then the calculations can be used to extract the dephasing time from the experimental data. This method allows the measurement of dephasing times considerably shorter than the pulse width.

The theory has been extensively discussed in References 6 and 12 . The problem is treated completely classically, using the nonlinear wave equation to describe the propagation of the light fields, and describing the vibrational medium as an ensemble of harmonic oscillators. The buildup of the vibrational amplitude is described by the integrodifferential equation: 12

$$
\frac{\partial Q\left(z, t^{\prime}\right)}{\partial z}=K_{1} K_{2} \int_{-\infty}^{t^{\prime}} d t^{\prime \prime} \exp \left[-\Gamma\left(t^{\prime}-t^{\prime \prime}\right)\right]\left|E_{L}\left(t^{\prime}\right)\right|^{2} Q\left(z, t^{\prime}\right)
$$

where $Q\left(z, t^{\prime}\right)$ is the vibrational amplitude at position $z$ and time $t^{\prime}$; $\Gamma=1 / T_{2} ; x_{1}=\left(8 \omega_{v 1 b} m\right)^{-1}(\partial \alpha / \partial q)$, where $\omega_{v i b}$ is the vibrational frequency and $\partial \alpha / \partial q$ is the differential polarizability; $\kappa_{2}=\left(\mu_{0} N / 4\right)(\partial \alpha / \partial q)\left(\omega_{s}{ }^{2} / \kappa_{s}\right)$, where $N$ is the molecular number dentiy; and $\left|E_{L}(t)\right|^{2}$ is the laser pulse 
Intensity envelope. The initial conditions are derived by assuming a constant Input Stokes field, $\mathrm{E}_{S}{ }^{\mathrm{O}}$, which represents the spontaneous emission background that becomes amplified through the stimulated gain process :

$$
Q\left(0, t^{\prime}\right)=1 \kappa_{1} E_{S}^{0} \int_{-\infty}^{t^{\prime}} d t^{\prime \prime} \exp \left[-\Gamma\left(t^{\prime}-t^{\prime \prime}\right)\right] E_{L}\left(t^{\prime \prime}\right)
$$

The quantity that is observed experimentally is the integrated antiStokes signal as a function of delay $t_{D}$, which is given by:

$$
I\left(t_{D}\right)=4 k_{j}{ }^{2} L^{2}\left[1+2|\Delta \vec{k}|^{2} L^{2}\right]^{-1} \int_{-\infty}^{\infty} d t^{\prime}\left|E_{P}\left(t^{\prime}-t_{D}\right)\right|^{2}\left|Q\left(t^{\prime}\right)\right|^{2}
$$

where $\kappa_{3}=\left(\mu_{0} N / 4\right)(\partial \alpha / \partial q), E_{P}(t)$ is the probe pulse envelope, and $Q\left(t^{\prime}\right)$ Is the vibrational amplitude at the end of the cell. The integral in Equation (48) is a convolution integral involving the probe pulse shape and the time-dependent vibrational amplitude. The calculation of $I\left(t_{D}\right)$ is performed in two steps: first Equation (46) is integrated numerically to obtain $Q\left(t^{\prime}\right)$ at the end of the cell, and then this function is squared and convoluted with the probe pulse shape to give finally $I\left(t_{D}\right)$.

Several details of this procedure should be pointed out: 1) In a coherent scattering experiment, the probe pulse and the exciting pulse have the same shape, since they are derived from the same $5300 \AA$ laser pulse by a beam splitter. 2) It has been found empirically that these pulses are described by a function of the form ${ }^{12}$

$$
E(t)= \begin{cases}\operatorname{sech}(t / C 2) & t>0 \\ \operatorname{Exp}(-t / C 1)^{2} & t<0\end{cases}
$$

The parameters $C_{1}$ and $C_{2}$ are all that is needed to define the pulse shape. 3) The factor $k_{1} k_{2}$ is related to the steady-state stimulated Raman gafn factor $\mathbf{G}_{\mathbf{s s}}$ by 


$$
G_{S S}=\kappa_{1} \kappa_{2} / T_{2}
$$

4) It is necessary to calculate only a normalized probe signal; thus the factor preceding the integral sign in (48) can be dropped.

A set of computer programs has been developed which carry out the numerical integration of (46), perform the convolution in (48), compute the statistical error between this calculated curve and a given set of experimental data, and produce a plot showing the calculated curve ana the experimental points. For convenience, the programs have been written to run on the LSI-11 microcomputer dedicated to the picosecond experiment. FORTRAN listings of all of these programs and instructions for their use are provided in Appendix 3.

The method used for the numerical integration is based on standard Runge-Kutta techniques. ${ }^{61}$ The Runge-Kutta formula for a general function $y^{\prime}=f(x, y)$ is

$$
y(x+h)=\frac{1}{6} h\left(\kappa_{1}+2 \kappa_{2}+2 \kappa_{3}+\kappa_{4}\right)+y(x)
$$

where $k_{1}=f(x, y), k_{2}=f\left(x+\frac{1}{2} h, y+\frac{1}{2} k_{1}\right), k_{3}=f\left(x+\frac{1}{2} h, y+\frac{1}{2} k_{2}\right)$, and $k_{4}=f\left(x+h, y+k_{3}\right)$. This requires four values of $f$ to be computed in order to calculate $y(x+h)$. Since (46) requires a numerical integration in two dimensions, the following procedure is used: ${ }^{12}$ an array if numbers is set up which represents the vibrational amplitude $Q$ as a function of time at some distance $z_{0}$ into the cell. The stimulated Raman cell is then divided into discrete steps along $z$, and the entire array $Q$ is stepped from one distance to the next using (51) element by element. This is done by first calculating an array representing $\partial Q / \partial z$ as a function of time by numerically performing the integral on the right-hand 
side of (46). This array is analogous to the first term on the righthand side of (51), except that it is a whole array and not just the value of a one-dimensional function. Performing three more integrations of (46) as indicated by (51) provides all of the information needed to step by one increment along $z$. This is done by applying (51) to each element of $Q$ as if that element contained the value of a one-dimensional function. Ditimately this procedure results in the function $Q(\ell, t)$, where $\ell$ is the total cell length. In practice, it was necessary to take 100-300 steps along $z$ in oraer to get the function $Q$ to within a few percent. 12

The function $Q(l, t)$ is squared and then convoluted with the probe Intensity profile as indicated by (48). The convolution is performed by simple brute force time-shifting and multiplication. It was found that a smooth curve could be obtained by taking only every fifth point in the calculated $Q^{2}$ and $E_{P}{ }^{2}$, resulting in a considerable savings in computer time.

These programs can be used in a systematic way to analyze experimental data. The first step is to evaluate the pulse shape parameters $C_{1}$ and $C_{2}$ in Equation (49). Two methods are available for doing this. The first is based on the fact that, when $T_{2}$ is very short, $Q\left(t^{\prime}\right)$ in the convolution integral (48) is a very sharply peaked function. It acts somewhat like a delta function, causing the function $I\left(t_{D}\right)$ to closely approximate the laser pulse shape. In other words, when the vibrational dephasing time is short, the probe scattered signal looks like a "picture" of the laser pulse, and the pulse shape is obtalned directly. The second method assumes that the correct value of $\mathrm{T}_{2}$ is known for one molecule out of several that have been studied. The computer programs are used to calculate probe scattering curves based on the known value of $\mathrm{T}_{2}$ and regarding the quantities $C_{1}$ and $C_{2}$ as variable parameters. By trial and 
error, one can then find the values of $C_{1}$ and $C_{2}$ which best reproduce the experimental curves. These values of $\mathrm{C}_{1}$ and $\mathrm{C}_{2}$ can then be used to fit the data on other molecules.

Once the pulse shape is known, the next step is to calculate a set of curves using a range of different $T_{2}$ values. These curves are stored in the computer, and are compared statistically with a set of experimental points. The value of $\mathrm{T}_{2}$ is then taken from that calculated curve which gives the best fit. This procedure somewhat compensates for the fact that no analytical expression exists for the probe scattered signal and that the standard type of least-square data analysis is not possible. This whole process is rendered considerably less laborious than it appears through the use of the RT-11 BATCH processor, which is part of the software available for the LST-11. BATCH is able to manipulate sequentially numbered files and execute a series of programs without intervention from the user. This makes it possible to calculate a set of 20-30 curves and perform a statistical comparison of each with 10-15 experimental data files overnight. An example of a BATCH listing which does this is also provided in Appendix 3.

A set of curves with different $T_{2}$ times calculated in this way is shown in Figure 32. Note that for values of $T_{2}$ comparable to or greater than the pulse width, the decaying wing of the curve is dominated by the decay of the vibrations and not the laser pulse. The fitting procedure just discussed is only necessary to estimate dephasing times that are shorter than the pulse width, in which case the wing of the curve represents the combined effects of the decaying pulse shape and the decaying vibrations. A set of experimental data, showing the best-fitting calculated curve, is given in Figure $33 .{ }^{62}$ 
Eigure 32. Calculated anti-Stokes signal for different dephasing times. The pulse shape was described by $\mathrm{Cl}=8.0$ and $\mathrm{C2}=5.0$.

Figure 33. Experimental data for the symetric C-H stretch in dimethyl sulfide 62 fit using the pulse shape used to calculate the curves in Figure 32. The best fit gives $T_{2}=4.5$. The Insert shows how the standard deviation of the data varies as a function of the value of $T_{2}$ input into the programs. 
CALCULATED PROBE SIGNAL VERSUS DELAY TIME

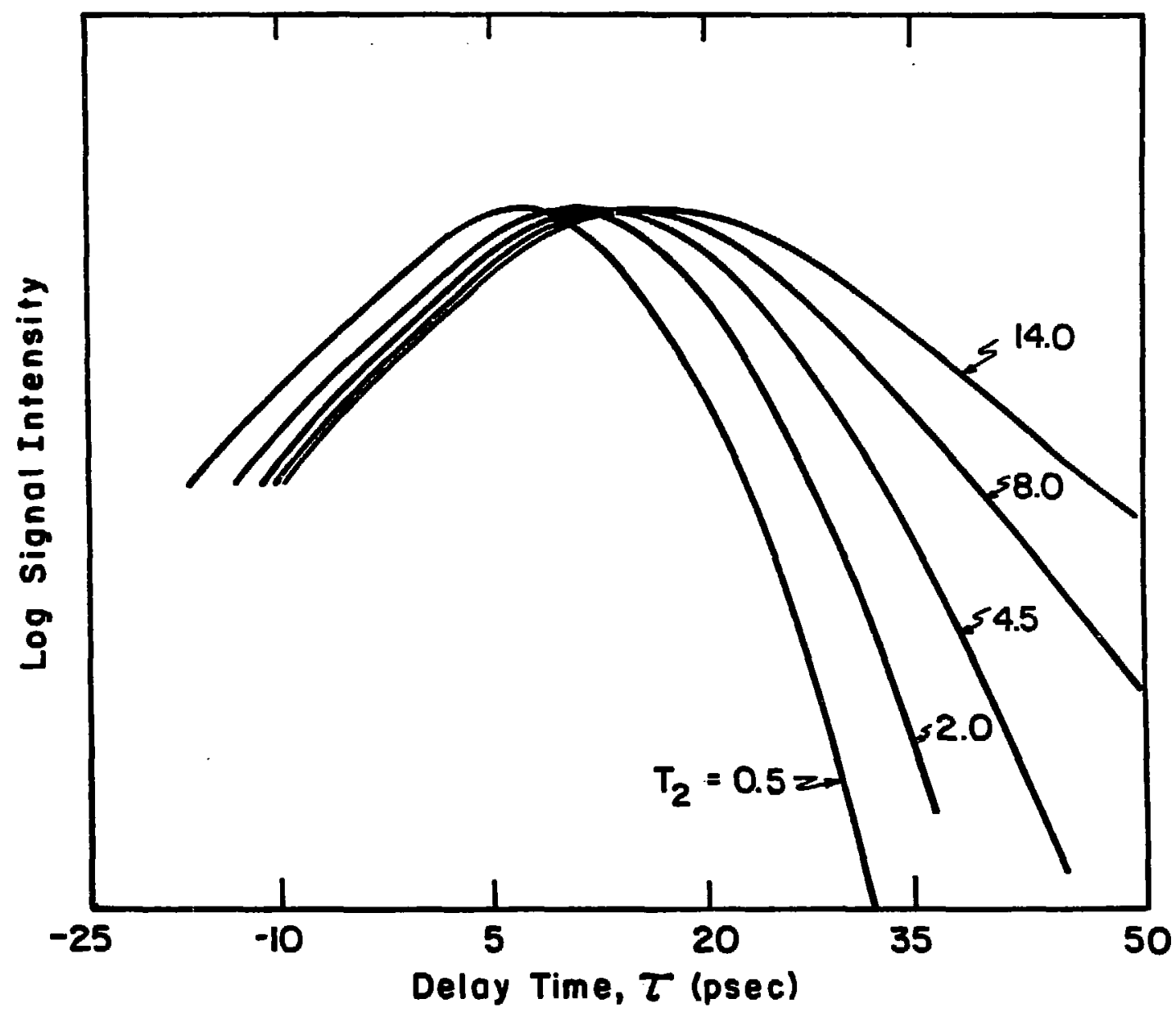

$X B L 805-5124$

Figure 32 
PROBE SCATTERING VERSUS DELAY TIME IN DIMETHYL SULFIDE

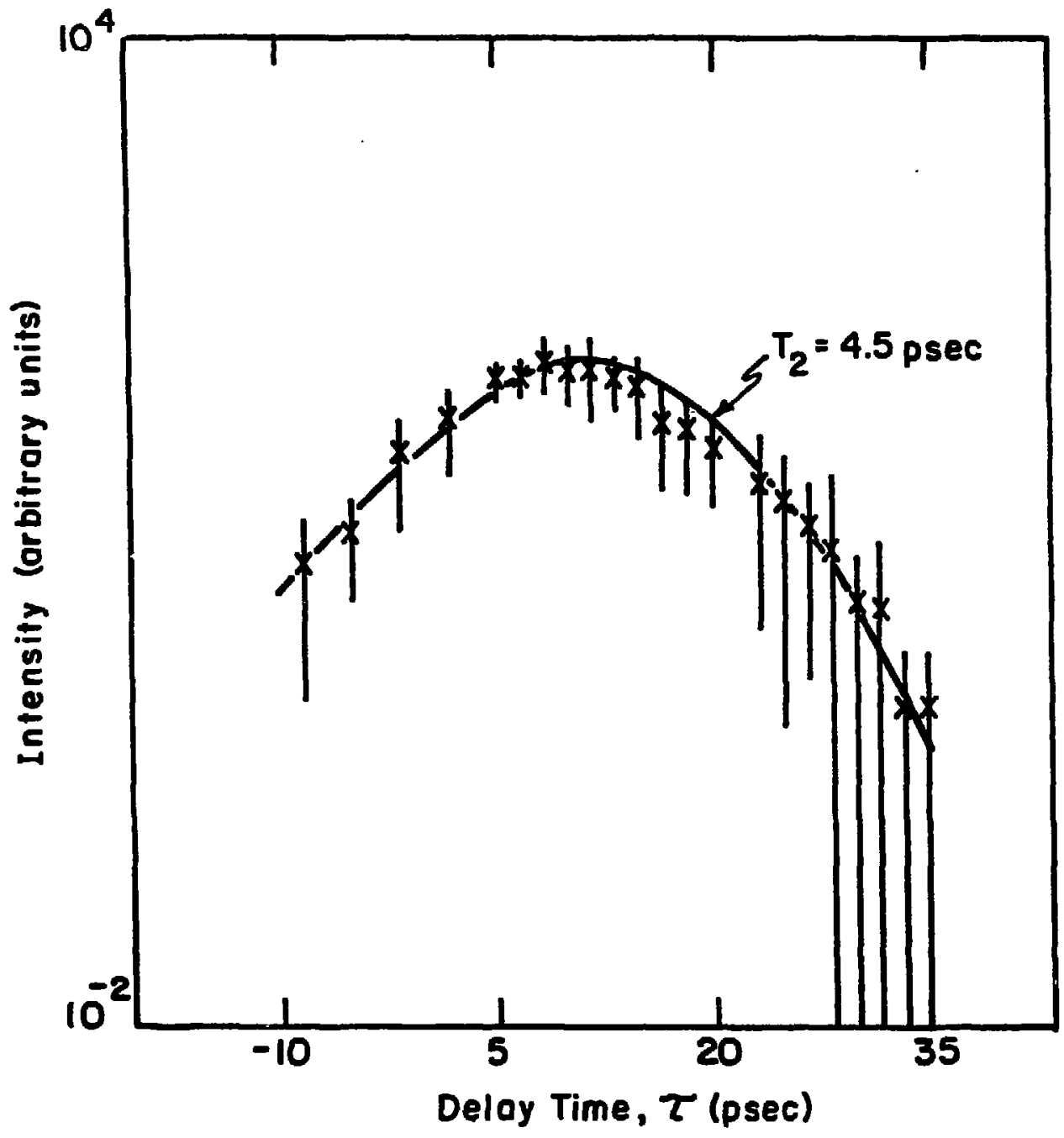

XBL 805-5123

Figure 33 
It should be mentioned that this procedure can be readily adapted to the case of inhomogeneous 1ines. The main difference occurs in Equation (46), which must be changed to

$$
\frac{\partial Q_{j}\left(z, t^{\prime}\right)}{\partial z}=k_{1} K_{2} \int_{-\infty}^{t^{\prime}} d t^{\prime \prime} \exp \left[-\Gamma_{j}\left(t^{\prime}-t^{\prime \prime}\right)\right]\left|E_{L}\left(t^{\prime \prime}\right)\right|^{2}\left[\sum_{m}\left(N_{m} / N\right) Q_{m}(z, t)\right]
$$

where $Q_{j}$ is the vibrational amplitude of a single component of the inhomogeneous Iine, $\mathrm{N}_{j}$ is the number density of the oscillators of that comporient, $N$ is the total number of oscillators, and $\Gamma_{j}=1 / T_{2}+i \Delta \omega_{j}$ where $\Delta w_{j}$ is the difference in frequency between the oscillators of component $j$ and the peak of the Inhomogeneous profile. Equation (48) for the probe scattering is changed by replacing $Q$ with a sum over those components $Q_{j}$ that are being probed (if selective wavevector matching is being used, not all components will contribute to the probe scattering). The problem with this calculation is that the number of integrals required goes up with the number of components in the Inhomogeneous distribution, and as a result of computer time limitations this calculation is impractical to do on a day-to-day basis. Figures 28 and 29 were calculated using the method just described, but for routine data analysis the calculation based on a single-component homogeneous line seems the best choice. One suggestion can be made to reduce the computational magnitude of the Inhomogeneous scattering calculation, although this has never been implemented. The first step is to perform the numerical integration of (46) using a value of $T_{2}$ which corresponds to the width of the total Inhomogeneous distribution; 1.e., the line is temporarily assumed to be homogeneous. The function $Q$ resulting from this calculation is taken as a first approximation to the quantity $\sum_{m}\left(N_{m} / N\right) Q_{m} 1 n(52)$. Each individual 
component $Q_{j}$ can be calculated from (52) by an integration along $z$ only, since the time integral on the right hand side has already been done in the first step. Once the individual components $Q_{j}$ have been calculated, they can be summed to compute a new $\sum_{m}\left(N_{m} / N\right) Q_{m}$, and the process repeated until convergence is achieved. 
CHAPTER V

SELF-PHASE MODULATION IN STTMULATED RAMAN SCATTERING

In the theory of stimulated Raman scattering described in the previous chapter, it was assumed that only one distinct mode of the molecule exhibits gain, and that the presence of the other vibrational modes may be neglected. For small molecules, this assumption is frequently justifled. For moderate sized molecules, such as xylene and durene, and for the exceptional case of methano1. 14 this assumption breaks down. In xylene, we have observed Stokes emission from the C-H stretch region that was extremely broad and Irreproducible from shot to shot; often radiation appeared $100 \mathrm{~cm}^{-1}$ or more from any Raman-active mode of the molecule. In methanol, both the symmetric and asymmetric C-H stretch modes have stimulated gain, ${ }^{14}$ and both peaks appear in the stimulated Stokes spectrum with roughly equal intensities. The stimulated spectrum of both xylene and methanol commonly exhibit a periodic progression of extra peaks, which is strongly suggestive that self-phase modulation is present. ${ }^{63-67}$ Existing theoretical descriptions of stimulated Raman excitation by picosecond pulses ${ }^{6-10,12,13}$ do not treat the effect of self-phase modulation in the presence of Stokes scattering from more than one mode. In this chapter, a theory is presented which describes the important features of phase-modulated, multiple mode stokes scattering.

The inclusion of self-phase modulation directly into the theory of stimulated Raman scattering, while possible in princlple, would lead to a problem of enormous computational complexity. A more practical approach was therefore employed, ${ }^{68}$ which consists of treating the phase modulation process separately. A general theory of phase-modulation was 
developed to describe the propagation of a light flejd consisting of any desired number of pulse shapes and frequencies. This phase-modulation theory could then be applied to a situation analogous to that encountered in transient stimulated Raman scattering, by choosing a light field which consists of a laser pulse and any number of Stokes pulses. The pulse shapes are calculated by the method discussed in the previous chapter. While this does not represent a rigorous description of stimulated gain in the presence of phase-modulation, the theory successfully explains the qualitative features of the spectra that we obse, Is addition, the separate theory of phase-modulation is of general interest and may find applications in problems not related to stimulated Raman scattering.

The propagation of a light field through matter is described by the Maxwell wave equation:

$$
\nabla^{2} \vec{E}-\frac{1}{c^{2}} \frac{\partial^{2} \vec{E}}{\partial t^{2}}=\frac{1}{c^{2}} \frac{\partial^{2} \vec{p}}{\partial t^{2}}
$$

The properties of the material determine the polarization $\overrightarrow{\mathbf{P}}$ by the relationship

$$
\overrightarrow{\mathbf{P}}=\overrightarrow{\vec{X}} \cdot \overrightarrow{\mathrm{E}}
$$

where the susceptibility $\overrightarrow{\vec{x}}$ is a complex, frequency-dependent second-rank tensor. ${ }^{68-70}$ The susceptibility completely describes, in classical terms, the optical properties of the medium. "Linear" optics, Including the effects of absorption, reflection, refraction, and so on, is described by a susceptibility $\vec{x}_{1}$ which is independent of the applied field. Nonlinear optics is described by expanding the susceptibility in powers of the applied field, namely: 


$$
\overrightarrow{\vec{x}}=\overrightarrow{\vec{x}}_{1}+\stackrel{\vec{t}}{\vec{x}}_{2} \cdot \overrightarrow{\vec{E}}+\stackrel{\vec{t}}{\vec{x}}_{3} \cdot \overrightarrow{\mathrm{E}} \cdot \overrightarrow{\mathrm{E}}+\ldots
$$

where each of the terms in the expansion is a successively higher rank tensor. From symetry arguments, $\chi_{2}$ vanishes in liquids and solids possessing a center of inversion symetry, 69,70 so for the purposes of this discussion $x_{3}$ is the lowest order nonlinearity of importance. For simplicity, it will be assimed that $x_{3}$ is a real scalar and that all fields are plane waves traveling in the z-direction. The susceptibility now assumes the form

$$
x=x_{1}+x_{3}|E|^{2}
$$

If higher powers of $\mathrm{E}$ are neglected. The index of refraction is defined by

$$
\mathbf{n}=(1+x)^{3 / 2}
$$

and $n$ can be expanded in powers of $E$ from (56) to give

$$
\mathrm{n}=\mathrm{n}_{0}+\mathrm{n}_{2}|\mathrm{E}|^{2}
$$

Equation (58) describes a situation in which the index of refraction depends on the intensity of the 1ight field. The effect of this can be understood by considering two waves, one propagating through a linear medium and the other through the nonlinear medium described by (58). The phase velocity is $\mathrm{c} / \mathrm{n}$; when the IInear wave has traveled a distance $z$ tts phase has changed by

$$
\phi_{\text {IInear }}=z \frac{c}{n_{0}}
$$


whereas the nonlinear wave has undergone a phase change of

$$
\phi_{\text {nonlinear }}=\frac{z c}{n_{0}+n_{2}|E|^{2}}
$$

The relative phase shift, $\phi$, of the nonlinear wave is then 63,64

$$
\phi=\operatorname{zen}_{2}|\mathrm{E}|^{2}=\mathrm{a}|\mathrm{E}|^{2} \text {. }
$$

If the time dependence of the linear field is represented by a pulse envelope $E_{0}(t)$ and a frequency factor $\exp (1 \omega t)$ :

$$
E_{\text {linear }}(t)=E_{0}(t) \exp \left(i \omega_{0} t\right)+\text { complex conjugate (c.c.) }
$$

then the nonlinear field must include the additional phase factor $\phi$ from (61):

$$
E_{\text {nonlinear }}(t)=E_{0}(t) \exp \left(i \omega_{0} t\right) \exp (i \phi i: i+c \cdot c .
$$

Note that $\phi$ is time dependent because $|E|^{2}$ in Equation (61) is time dependent.

The spectrum of the nonlinear field is the square modulus of the Fourier transform of (63):

$$
\begin{aligned}
I(\omega) & =\left|\int_{-\infty}^{\infty} \exp (1 \omega t) E_{\text {nonlinear }}(t)\right|^{2} \\
& =\left|\int_{-\infty}^{\infty} \exp (1 \omega t) \exp \left(1 \omega_{0} t+1 \phi(t)\right) E_{0}(t) d t\right|^{2}
\end{aligned}
$$

This equation has been used previously to describe the effect of self-phase modulation in a variety of systems. ${ }^{63-67}$ Additional pulses of arbitrary shape and frequency can be formally incorporated into the spectrum by changing (62) to read: 


$$
E_{\text {linear }}(t)=\sum_{I} E_{i}(t) \exp \left(i w_{i} t\right)+c \cdot c .
$$

and making (63) read:

$$
E_{\text {nonlinear }}(t)=E_{\text {linear }}(t) \exp (1 \phi(t))
$$

Frow (65)

$$
\begin{aligned}
|E|^{2} & =\sum_{i} \sum_{j} E_{i}(t) E_{j}(t) \exp \left(1\left[\omega_{i}+\omega_{j}\right] t\right)+c . c . \\
& +\sum_{1} \sum_{j} E_{i}(t) E_{j}{ }^{*}(t) \exp \left(i\left[\omega_{i}-\omega_{j}\right] t\right)+c \cdot c .
\end{aligned}
$$

For the purposes of illustration, consider one term in this expression for $|E|^{2}$, say $E_{1}(t) E_{2}{ }^{*}(t) \exp \left(1\left[\omega_{1}-\omega_{2}\right] t\right)$. Using (61) to substitute for $\phi(t)$ In (66) gives

$$
\begin{aligned}
E_{\text {nonlinear }}(t) & =E_{1 \text { inear }}(t) \exp \left[\operatorname{laE}_{1}(t) E_{2}{ }^{*}(t) \exp \left(i\left[\omega_{1}-\omega_{2}\right] t\right)\right] \\
& =E_{11 \text { near }}(t) \exp \left[A(t) \exp \left(i\left[\omega_{1}-\omega_{2}\right] t\right)\right] .
\end{aligned}
$$

Expanding the exponential:

$$
\begin{gathered}
E_{\text {nonlinear }}(t)=E_{11 \text { near }}\left(t ; A(t) \exp \left(i\left[\omega_{1}-\omega_{2}\right] t\right)\right. \\
+\frac{1}{2} A^{2}(t) \exp \left(21\left[\omega_{1}-\omega_{2}\right] t\right) \\
+\ldots\} .
\end{gathered}
$$

When this is inserted 1nto (64) and the Fourier transform computed, the first term in the bracket will generate a peak at a frequency of $\omega_{0}+$ $\omega_{1}-\omega_{2}$ (where $\omega_{0} 1 s$ the frequency of a peak in the spectrum of $E_{1 \text { inear }}$ ); the second term in brackets generates a peak at $\omega_{0}+2\left(\omega_{1}-\omega_{2}\right)$, and so on. In other words, the phase modulation of the field (65) generates a series of "satellite" peaks around the parent peak at $\omega_{0}$; the satellite peaks are separated from each other by the frequency difference between a pair 
of peaks in the spectrum of $E_{1 \text { inear }}$

It is useful to distingulsh the two manifestations of phase-modulation which emerge from the foregoing discussion. The first is described by Equations (62) and (63), and is the only effect present in fields containIng InItially one frequency component. This phase modulation produces frequency shifts $\Delta(t)$ which are proportional to the time derivative of the nonlinear phase factor $\phi:^{64}$

$$
\Delta(t)=\frac{\partial}{\partial t} \phi(t)=a \frac{\partial}{\partial t}|E(t)|^{2}
$$

From (61):

$$
|E(t)|^{2}=\left|E_{0}(t)\right|^{2}+E_{0}^{2} \exp \left(21 \omega_{0} t\right)+c \cdot c .
$$

Near $w=w_{0}$, only the first term in (71) is important, so that combining (70) and (71) gives

$$
\Delta(t)=a \frac{\partial}{\partial t}\left|E_{0}(t)\right|^{2}
$$

This equation means that the nonlinear Index produces an instantaneous frequency shift which is proportional to the derivative of the pulse Intensity envelope. This effect, which is also present in fields of more than one component, w111 be termed "envelope modulation."

The second type of phase-modulation is described by (65) and (66). As already discussed, the appearance of the frequency factors $\exp \left(i\left[w_{i}-w_{j}\right] t\right)$ generates new satellite components separated by $w_{i}-w_{j}$. This effect, which appears only in multiple component fields, is independent of the exact shape of the pulse envelope and will be termed "satellite modulation." 
To 1llustrate these ideas, a serles of computer calculations was performed. 71 In each case, an input pulse was chosen with the general form of $E_{\text {linear }}$ in Equation (65). Equation (61) could then be used to calculate $\phi(t)$. $E_{\text {linear }}$ and $\phi(t)$ were substituted into (66) to generate $E_{\text {nonlinear, whose Fourier transform (64) was taken using a standard Fast }}$ Fourier Transform ${ }^{72}$ routine. The resulting spectrum was plotted over the desired frequency range.

The calculation is begun by storing the input pulse in the computer as a one-dimenstonal array of complex numbers. To do this, the pulse shape $E_{1}(t)$ is first calculated as an array of real numbers, which is then multiplied by a complex frequency factor exp $\left(i w_{i} t\right)$. Multiple component Input pulses are constructed by simply adding together the arrays representing the individual components, making it possible to vary the Intensity and peak position of each component In any desired way. It should be noted that the product of the nonlinear constant a in Equation (61) and the pulse peak intensity form a single parameter In these calculations, which will be denoted $\phi_{\text {peak. Changes in the values }}$ of $z$ and $n_{2}$ have the same effect as changes in the pulse intensity; the values of all of these quantities can thus be accounted for by this single parameter $\phi_{\text {peak }}$. Throughout these calculations, the dispersion of $\mathbf{n}_{2}$ was neglected.

An additional feature of the computer programs can be used to delete one of the components of the input pulse just before taking the Fourier transform. The pulse w111 not appeer in the spectrum, but is included in $E(t)$ for the purpose of calculating the nonlinear phase factor $\phi$. This corresponds to the experimental situation when a stimulated Raman epectrum is belng taken, since the laser pulso may contribute to the 
nonlinear index of refraction, but is not observed since it is thousands of wavenumbers from the Stokes wavelength.

Using these methods, the effects of varying the pulse envelope, the number and distribution of frequency components, and the intensities of each was systematically investigated. Three different pulse envelopes were used: 1) gaussian, 2) a typical Nd:glass laser pulse shape of the type described by Equation (49), and 3) a Stokes pulse shape calculated numerically by the methods described in Chapter IV. The results of the calculations are 1llustrated by F1gures 34 and 35 , and show the two contributions to the modulation discussed above. Envelope modulation appears in every case, but does not become important until $\phi_{\text {peak }} \geq 10$. Satellite modulation, on the other hand, generates the peaks wh1ch grow in Figure $35(f)-(h)$, and visibly affects the appearance of the two-component spectrum at $\phi_{\text {peak }}$ values $\geq 0.1$. The fact that satellite modulation appears at such low intensities indicates that multiple component Stokes spectra should more readily show the effects of phase modulation than single component spectra.

The quantitative comparison of this theory with experiments is difficult because the nonlinear index $n_{2}$ and its dispersion are known for only a few simple liquids. ${ }^{73}$ In addition, it is difficult to know the intensities of the pump and Stokes fields Inside the sample cell. But the qualitative predictions of the theory are tr.stable, and were confirmed by a study of the stimulated Raman spectrum of methanol. Frequency doubled Nd:glass laser pulses of $10 \mathrm{~mJ}$ were focussed into a $15 \mathrm{~cm}$ cell of methanol, and the resulting Stokes spectra were displayed on an optical multichannel analyzer. By varying the pulse energy and focus, a range of phase-modulation effects could be produced. It is clear from the spectra presented in Figures 36 and 37 that the features which appear 
Figure 34. Envelope modulation of single-component pulses. In (a)-(d), a gaussian pulse shape was used; in (e)-(h) a Nd:glass laser exciting pulse propagates with a Stokes pulse, and the Stokes spectrum as a function of laser pulse intensity is plotted. The nonlinear refractive index in $(e)-(h)$ is generated by the laser pulse, and the Stokes spectrum displays the effects of the modulation. $\phi_{\text {peak }}$ values are: (a) 0.0 (b) 0.9 (c) 10.0 (d) 90.0 (e) 0.1 (f) 3.6 (g) 40.0 (h) 250.0 . The asymetry in $(e)-(h)$ results from the asymetry of the pulse shapes.

Figure 35. These spectra are calculated for the case of a laser pulse and a two-component Stokes pulse. The Stokes spectrum in the absence of phase-modulation is shown in (e). Curves (e)-(h) represent a fixed laser Intensity and increasing Stokes intensity; the values of $\phi_{\text {peak }}$ are (e) 0.0 (f) 0.1 (g) 0.4 (h) 2.5. In (a)-(d) the Stokes field of (f), 1.e., $\phi_{\text {peak }}=$ 0.1 , was held fixed as the laser intensity was increased. Values of $\phi_{\text {peak }}$ for the laser field are (a) 0.1 (b) 10.0 (c) 40.0 (d) 250.0 . The calculation shows clearly that the sidebands which grow in curves $(f)-(h)$ are related to the presence of two stokes components. They appear at intensities too low to cause significant modulation of a single peak, and are separated in frequency by the splitting between the two parent Stokes peaks.

Figure 36. Spectrum (a) is the transient stimulated Raman spectrum of methanol in the absence of self-phase modulation, and shows the two peaks corresponding to the two Raman-active $\mathrm{C}-\mathrm{H}$ stretch modes of this molecule. In (b) and (c) the sidebands resulting from phase-modulation are shown, as illustrated by the equally spaced arrows. These spectra resemble the calculated spectra Fig. $35(\mathrm{~g})$ and $35(\mathrm{~h})$. In (d) a large amount of broadening is also present, partially obscuring the parent peaks and the periodic sideband structure. 
Figure 37. The spectrum on the right was obtained by tight focussing of the pump laser pulse. The arrows indicate the peak positions in the unmodulated methanol spectrum. The calculated spectrum on the left is almost identical to Fig. $34(g)$; its resemblance to the experimental spectrum indicates the presence of envelope modulation at high light field intensities. 
CALCULATEd single Component phase modulated spectra

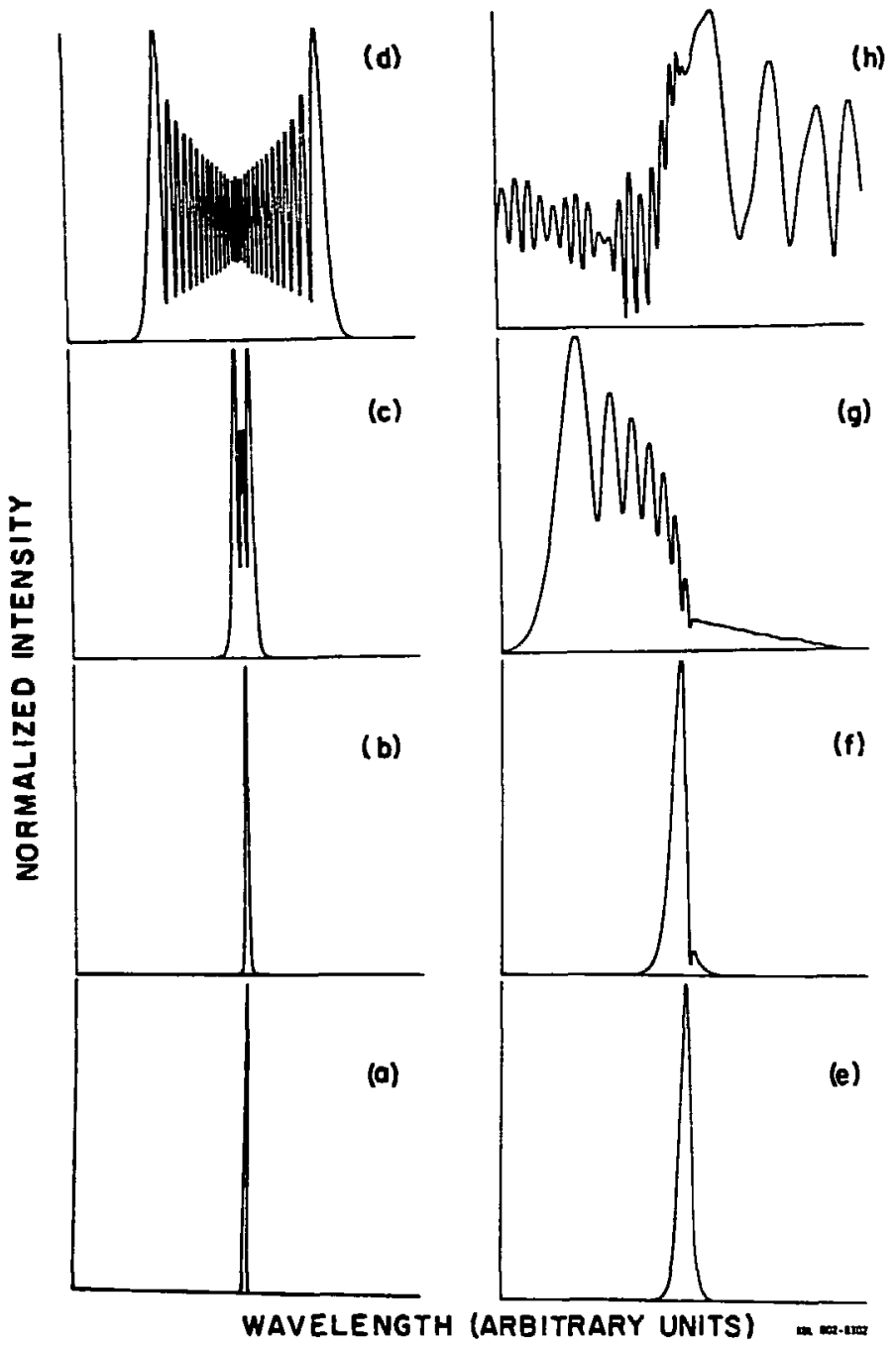

Figure 34 
CALCULATED TWO-COMPONENT PHASE MODULATED SPECTRA

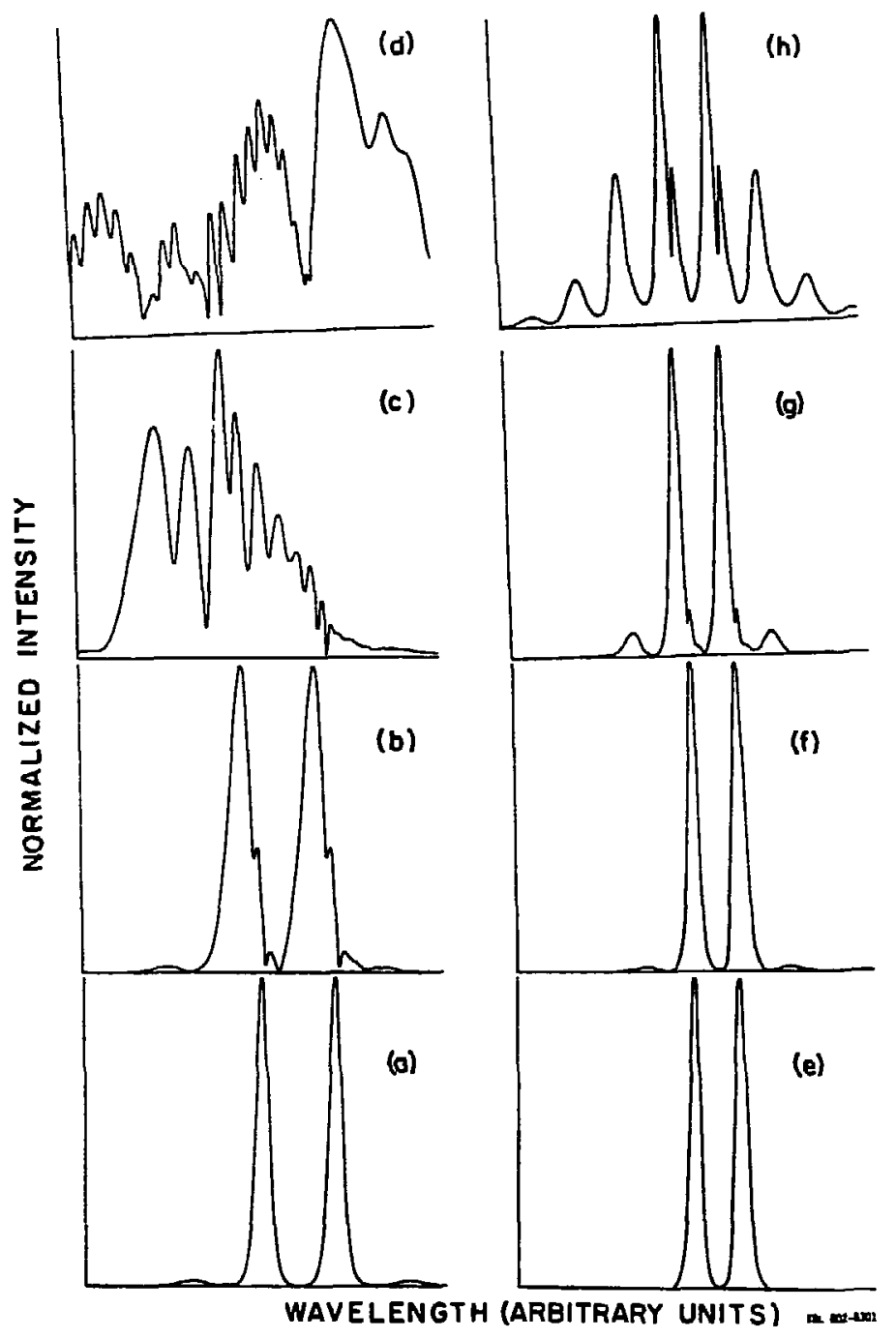

F1gure 35 


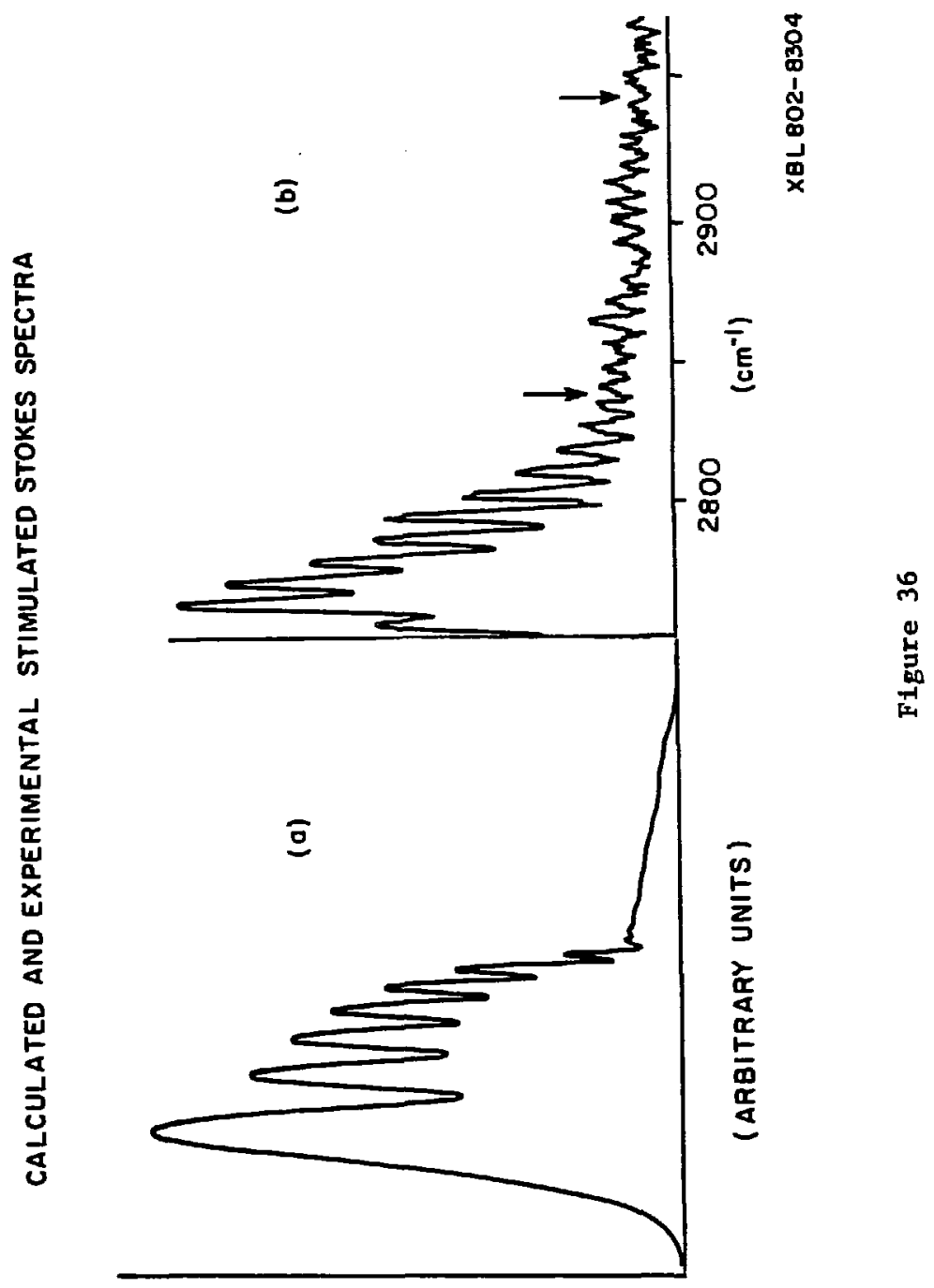

ALISN $3 \perp N I O \exists Z I 7 \forall W Y O N$ 


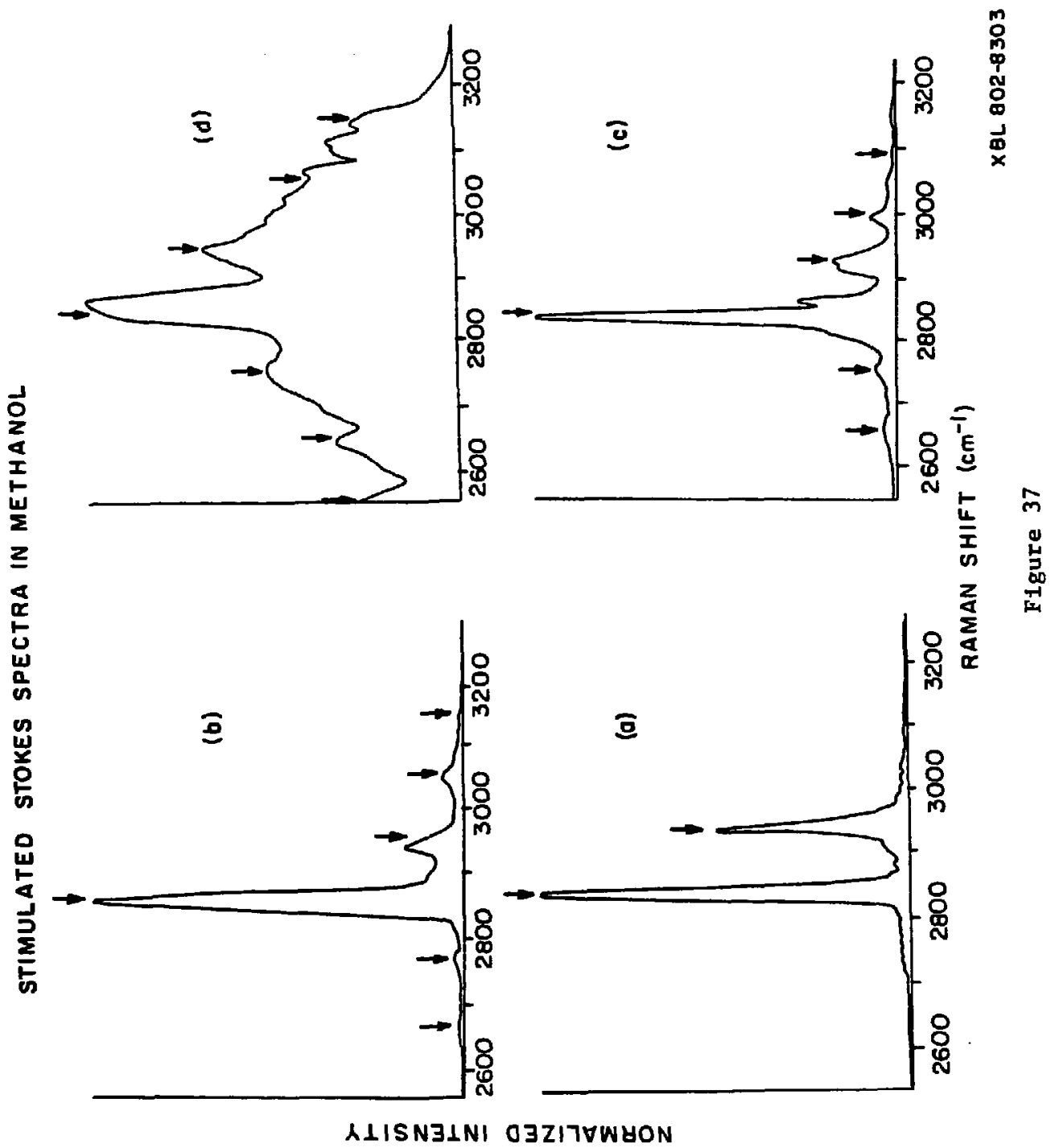


are explained quite well by the calculated spectra in Figures 34 and 35. The satellite peaks which appear in Figures $36(b)$ and (c), and the extremely modulated spectra in Figures $36(d)$ and 37 are characteristic.

The fact that the onset of satellite modulation occurs at relatively low intensitfes suggests that molecules having spontaneous Raman spectra consisting of numerous close or overlapping bands should show very complex stimulated spectra. We have observed this in xylene and durene, and other workers have reported similar effects (without explanation). ${ }^{74}$ The theory developed here should make possible the interpretation of such complicated spectra, and even offers the possibility of studying those molecular properties known to be related to nonlinear optical constants. 66,75 At the very least, this theory serves as a warning to the picosecond spectroscopist tha' moderately sized molecules may of ten exhibit severe broadening of their stimulated Raman spectra. When stimulated scattering occurs from more than one mode, phase-modulation may be an intrinsic part of the excitation process. 


\section{CHAPTER VI}

\section{CONCLUSIONS AND PROSPECTS}

It could be sald that the study of vibrational energy transfer and dephasing in condensed phases is in its infancy. Neither of the experimental techniques used in the present work is ideal, and the theoretical literature hardly shows anything like a consensus on how this problem can be modelled.

But in spite of these facts, definite progress has been rade. The work on durene has shown that spontaneous Raman spectroscopy can be a powerful tool, especially when interesting temperature-dependent effects are present in the spectrum. The exchange model which we have developed explains the spectrum of durene quite well, and even reveals insights Into the importance of delocalization in vibrational modes in solids. Perhaps the most intriguing aspect of the application of exchange theory is that a great deal of information about the behavior of low frequency modes can be extracted from a study of the high frequency modes. It is because of the simplicity of the coupling scheme - one high frequency mode coupled to just one low-frequency mode - that the parameters which result from the exchange analysis can be interpreted in this way. The fact that the data establishes the validity of the simple coupling scheme in the vast majority of cases is one of the most important points in favor of the theory. The successful interpretation of the durene data does not establish the ubiquity of the exchange mechanism. But recent applications of the theory to other types of spectroscopy 41,51 are encouraging, and suggest that further work along these lines may well prove fruitful. 
The picosecond work has succeeded in demonstrating the possibilities and Ifmitations of these kinds of experiments. Studying solids in this way is extremely difficult, and vibrational modes that have no stimulated Raman gain are Inaccessible by this method. For some molecules, self-phase modulation may prove a roadblock. Nevertheless, a means of measuring homogeneous dephasing times is valuable, even if 1ts range of application $1 \mathrm{~s}$ limited. The recent measurement of a series of dephasing times in simple methyl liquids ${ }^{11}$ indicates the potential future directions open to this technique, and also shows that enough suitable molecules can be found to outline general trends.

Since the plcosecond methods are mainly applicable to 11quids, and the exchange analysis requires a temperature range which is generally avallable only in the solid, the two techniques together may provide an Interesting Insight into how vibrational dynamics are different (or similar) in the two phases. Investigating the same molecule by both methods, perhaps including a picosecond $T_{1}$ measurement, offers the possiblifty of a further test of exchange theory. If it is found that the exchange mechanism is important in the liquid state as we11, the combination of the two experimental techniques may lead to an even more thorough understanding of the vibrational dynamics than has been possible before.

Chapter I ralsed the general question of what concepts could be used to describe energy and phase transfer in condensed matter. The continued development of experimental methods will enable the testing of the models constructed in answer to this question. Exchange theory represents one such model, and the development, testing, and application of that theory can be expected to lead to further generalizations about the nature of molecular dynamics. 


\section{APPENDIX 1}

\section{INTERMEDIATE STEP IN EXCHANGE THEORY DERIVATION}

We wh to take the time average of the following function (text Equation (10)):

$$
\phi(\tau)=\left\langle\exp \left(1 \int_{t}^{t+\tau} \Delta \omega_{01}(t) d t\right)\right\rangle
$$

The interval $t$ to $t+\tau$ is broken up into $n$ equal subintervals. The Integral is replaced by a summation, and the varlables by discrete indices:

$$
\phi(\tau)=\left\langle\exp 1 \sum_{m=0}^{n} \frac{\tau}{n} \Delta \omega\left(t+\frac{m}{n} \tau\right)\right\rangle .
$$

We define a quantity $\Delta \omega_{m}$ by

$$
\Delta \omega_{\mathrm{m}}=\Delta \omega\left(t+\frac{\mathrm{m}}{\mathrm{n}} \tau\right)
$$

which represents the value of $\Delta \omega(t)$ at the end of each subinterval m. We now assume that $\Delta \omega(t)$ can have only one value on a given subinterval, which can be elther $-\delta \omega / 2$ or $\delta w / 2$ for the case of a two level system. As the Index $m$ is stepped from 0 to $n$, a set of $\Delta \omega_{m}$ values results which represents the "path" the system took to get from $t$ to $t+\tau$. We now proceed by computing the probability that a particular set of $\Delta w_{m}$ values occurs, and replace the time average in (75) by a probability-weighted average over all possible sets of $\Delta \omega_{m}$. We make the following definitions:

$$
\begin{aligned}
& W_{+}=\text {probability that } \Delta \omega_{\mathrm{m}}=-\delta \omega / 2 \text { and that } \\
& \Delta \omega_{m+1}=\delta \omega / 2 ; 1 . e ., \text { an upward transition probability. } \\
& 1-w_{+}=\text {probab1l1ty that } \Delta \omega_{\mathrm{m}}=-\delta \omega / 2 \text { and that } \\
& \Delta \omega_{m+1}=-\delta \omega / 2 \text {. }
\end{aligned}
$$




$$
\begin{aligned}
& \mathrm{w}_{-}=\text {probability that } \Delta_{\mathrm{m}}=\delta \omega / 2 \text { and that } \\
& \Delta \omega_{\mathrm{m}+1}=-\delta \omega / 2 ; 1 . e ., \text { a downward transition probability. } \\
& 1-\mathrm{w}_{-}=\text {probability that } \Delta \omega_{\mathrm{m}}=\delta \omega / 2 \text { and that } \Delta \omega_{\mathrm{m}+1}=\delta \omega / 2 . \\
& \mathrm{w}_{1}(-\delta \omega / 2)=\text { probability that } \Delta \omega_{0}=-\delta \omega / 2 . \\
& w_{1}(\delta \omega / 2)=\text { probability that } \Delta \omega_{0}=\delta \omega / 2 .
\end{aligned}
$$

Consider the case where $n=1$. In this case, the summation in (74) will have only two terms

$$
\phi(\tau)=\langle\exp 1 \tau[\Delta \omega(t)+\Delta \omega(t+\tau)]\rangle
$$

The function $\Delta \omega(t)+\Delta \omega(t+\tau)$ has four possible values, whose probabilities can be computed from the W's defined above. The angle brackets in (76) w11 be replaced by these four terms, each multiplied by its probab1lity:

$$
\begin{aligned}
\phi(\tau) & =W_{1}(-\delta \omega / 2) \exp (-i \tau \delta \omega / 2)\left(1-W_{+}\right) \exp (-1 \tau \delta \omega / 2) \\
& +W_{1}(-\delta \omega / 2) \exp (-i \tau \delta \omega / 2) W_{+} \exp (1 \tau \delta \omega / 2) \\
& +W_{1}(\delta \omega / 2) \exp (i \tau \delta \omega / 2)\left(1-W_{-}\right) \exp (1 \tau \delta \omega / 2) \\
& +W_{1}(\delta \omega / 2) \exp (i \tau \delta \omega / 2) W_{-} \exp (-i \tau \delta \omega / 2) .
\end{aligned}
$$

This can be rewritten in matrix form:

$$
\begin{aligned}
& \phi(\tau)=\left(W_{1}(-\delta \omega / 2) W_{1}(\delta \omega / 2)\right)\left(\begin{array}{cc}
\exp (-i \tau \delta \omega / 2) & 0 \\
0 & \exp (i \tau \delta \omega / 2)
\end{array}\right) \\
& x\left(\begin{array}{cc}
1-W_{+} & W_{+} \\
W_{-} & 1-W_{-}
\end{array}\right)\left(\begin{array}{c}
\exp (-1 \tau \delta \omega / 2) \\
\exp (i \tau \delta \omega / 2)
\end{array}\right)
\end{aligned}
$$

For $n=2$, the first term in the expansion along the lines of (77) looks like: 


$$
\begin{gathered}
W_{1}(-\delta \omega / 2) \exp (-1 \tau \delta \omega / 4)\left(1-W_{+}\right) \exp (-i \tau \delta \omega / 4)\left(1-W_{+}\right) \exp (-i \tau \delta \omega / 4) \\
=w_{1}(-\delta \omega / 2) \exp (-i \tau \delta \omega / 4)^{2}\left(1-W_{+}\right)^{2} \exp (-1 \tau \delta \omega / 2) .
\end{gathered}
$$

In matrix form, this would square the two matrices in the middle of (78). For $n=N(78)$ becomes

$$
\begin{gathered}
\phi(\tau)=\left(W_{1}(-\delta \omega / 2) W_{1}(\delta \omega / 2)\right)\left(\begin{array}{cc}
\exp \left(-i \frac{\tau}{N} \delta \omega / 2\right) & 0 \\
0 & \exp \left(1 \frac{\tau}{N} \delta \omega / \tau\right)
\end{array}\right)^{N} \\
\times\left(\begin{array}{cc}
1-W_{+} & W_{+} \\
W_{-} & 1-W_{-}
\end{array}\right)\left(\begin{array}{l}
\exp \left(-1 \frac{\tau}{N} \delta \omega / 2\right) \\
\exp \left(1 \frac{\tau}{N} \delta \omega / 2\right)
\end{array}\right)
\end{gathered}
$$

Define the matrices $w_{1}, \exp \left(1 \frac{\tau}{N} \Delta \omega\right), w$, and $\exp \left(1 \frac{\tau}{N} \Delta \omega\right)$ by rewriting $(80)$ :

$$
\phi(\tau)=\underbrace{W}_{1} \cdot\left(\exp \left(1 \frac{\tau}{N} \Delta \omega\right) \underset{\sim}{W}\right)^{N} \exp \left(1 \frac{\tau}{N} \underset{\sim}{\Delta \omega}\right)
$$

Let $\exp \left(1 \frac{\tau}{N} \Delta \omega\right)=1+1 \frac{\tau}{N} \Delta \omega$ and $W=1+\pi \frac{\tau}{N} ;$ let $N+\infty$

$$
\phi(\tau)=\omega_{1} \cdot \exp \tau(\underset{\sim}{\Delta \omega}+\pi) \cdot \underset{\sim}{1} .
$$




\section{APPENDIX 2}

EXCHANGE THEORY FROM ZWANZIG-MORI FORMALISM

In the Zwanzig-Mori formalism, the motion of a dynamical variable $A$ is described by an equation of the form ${ }^{30}$

$$
\frac{d}{d t} A(t)-i \omega_{0} A(t)=\int_{0}^{t} \phi(i-s) A(s) d s+f(t)
$$

where $\omega$ is the frequency, $f(t)$ is a random force acting upon $A$, and $\phi$ is a damping function related to $f(t)$ by the fluctuation-dissipation theorem:

$$
\phi(t)=\langle f(t) f *(0)\rangle .
$$

The brackets represent ensemble averages. In order to describe the motion of more than one dynamical variable, Equation (83) can be regarded as a matrix equation. The solution of (84) is

$$
A(t)=\equiv(t) A(0)+\int_{0}^{t} \equiv(t-s) \cdot f(s) d \cdot s
$$

where the correlation function $\Xi(t)$ is defined in terms of its Fou: ier transform:

$$
\Xi(w)=\frac{1}{w I-w_{0}-I \hat{\phi}(w)}
$$

and

$$
\hat{\phi}(\omega)=\int_{0}^{\infty} \exp (-1 \omega t) \phi(t) d t
$$

The problem is reduced to the evaluation of $\phi(\omega)$ according to (84), 1.e., finding the Fourler transforms of the force autocorrelation functions. This is the approach employed by Wertheimer ${ }^{18-21}$ in treating vibrational dephasing in condensed systems. 
The form of (86) requires an approximation to be made in the case where the quantities $A, \phi, f$, and $w_{0}$ are matrices, namely, the matrix $\phi$ must be diagonalized. This is accomplished using Rayleigh-Schroedinger perturbation theory, which results in new matrix elements $\phi_{1}^{\prime}$ given by

$$
\phi_{i}^{\prime}=\phi_{i 1}+\sum_{i=j} \frac{\phi_{1 j} \phi_{j 1}}{\phi_{i i}-\phi_{j j}}
$$

where $\phi_{1 j}(t)=\left\langle f_{1}(t) f_{j} *(0)\right\rangle$ and $f_{i}(t)$ is the random force on dynamical variable $A_{i}$.

In the Markoff approximation, it is assumed that $\phi_{1 j}(t)=$ constant $x \delta(t)$ and hence $\phi_{i j}(\omega)=$ constant. The exchange theory of Harris 16,17 can be readily derived by considering two modes: $A_{1}$ with resonance frequency $\omega_{0}$, and $A_{2}$ with resonance frequency $\omega_{0}+\Delta \omega$. The quantity characterizes the linewidth in the absence of dephasing, $\tau^{-1}$ is the depopulation rate of mode 2 , and the quantity $\sigma=\exp \left(-E_{i} / k T\right)$ is introduced in the ensemble averaging process to satisfy detailed balance:

$$
\begin{aligned}
\phi_{11}(\omega) & =\Gamma+\sigma \tau^{-1} \\
\phi_{22}(\omega) & =\Gamma+\tau^{-1} \\
\phi_{21}(\omega) & =\sigma \tau^{-1} \\
\phi_{12}(\omega) & =\tau^{-1} \\
\omega_{11} & =\omega_{0} \\
\omega_{22} & =\omega_{0}+\Delta \omega .
\end{aligned}
$$

Combination of (89) with (88) and (86) gives, in the low temperature 1imit $\sigma \ll 1$ :

$$
\equiv(\omega)=\frac{1}{\omega 1-L}
$$


where the matrix $L$ is diagonal. The lineshape of the transition $A_{1}$ can be described by calculating $\mathrm{L}_{1}$ :

$$
L_{1} \text { (exchange) }=\omega_{0}+\Gamma-1 \sigma \frac{\Delta \omega \tau^{-1}}{i \Delta \omega+\tau^{-1}} .
$$

Using the fact that $\operatorname{Re}(L)=$ frequency and $\operatorname{Im}(L)=$ damping, we obtain

$$
\begin{aligned}
& \operatorname{Re}(L)=\omega_{\text {eff }}=\omega_{0}+\frac{\sigma \tau^{2}(\Delta \omega)^{2}}{1+(\Delta \omega)^{2} \tau^{2}} \\
& \operatorname{Im}(L)=\Gamma_{\text {eff }}=\Gamma+\frac{\sigma \tau \Delta \omega}{1+(\Delta \omega)^{2} \tau^{2}}
\end{aligned}
$$

which are the same as Equations (27) and (29).

A more careful and comprehensive development along these lines sheds further light on the meaning of the parameters in (92) and (93), and allows the inclusion of additional dephasing and relaxation mechanisms. This more careful approach differs from the derivation above in three important respects: 1) The presence of more than one pair of exchanging modes can be readily represented. 2) Additional dephasing mechanisms are included by adding more terms to the force autocorrelation functions (84). Any physical process whose contribution is to be considered can be described by choosing an appropriate functional form for $\phi(t)$. 3) The Markoffian approximation need not be invoked; instead, Wertheimer employs a "quasi-Markoffian" approximation ${ }^{21}$ in which $\phi(\omega)$ is a complex constant $\phi_{a}$ near a resonance of interest $\omega=\omega_{a}$. In other words, $\phi(\omega)$ Is still considered to be a constant, as in the Markoffian case, but the value of the constant depends upon the frequency of a nearby resonance $\omega_{a} \cdot$

The treatment of Reference 21 includes the effects of exciton transfer processes in both the low-frequency and high-frequency modes, 
the effects of dynamic coupling between a given high-frequency mode and nelghboring modes (this is particularly important when Fermi resonances are present), the effects of cross-correlations, and the effects of relaxation of the low-frequency modes. It is shown ${ }^{21}$ that when onl: one pair of modes is important, Equation (91) is still the correct functional form to describe the lineshape, but that the quantities $\Delta \omega$ and $\tau$ have Imaginary components. The presence of these components, denoted by $\Delta \omega^{\prime}$ and $\tau^{\prime}$ respectively, produces a dephasing contribution which differs physically from exchange, and is described by

$$
\begin{gathered}
L_{1}(\text { dynamic }) \cong \omega_{0}+1 \Gamma-\sigma\left[\frac{\tau^{\prime-1} \Delta \omega}{\left(\tau^{\prime-1}-\Delta \omega\right)^{2}+\left(\tau^{-1}-\Delta \omega^{\prime}\right)^{2}}\right] \\
\left.x\left[\tau^{\prime-1}-\Delta \omega\right)-1\left(\tau^{-1}-\Delta \omega^{\prime}\right)\right]
\end{gathered}
$$

Two important conclusions can be drawn from this equation. The first stems from the consideration that $\tau^{-1}$ is, according to the discussion of References 17-21, temperature-dependent. Since the frequency shift from (94) is proportional to $\left(\tau^{-1}-\Delta \omega\right)$, the possibility exists that the direction of the frequency shift could reverse with increasing temperature. We have never observed such behavior experimentally. In any case, the ratio of the broadening to the shift, which is temperatureindependent in the HSC model, is not expected to be temperature independent in the dynamic coupling treatment. The second conclusion concerns the coupling of high- to low-frequency modes. From (94) we would anticipate that a given high-frequency mode would be coupled most strongly to that low-frequency mode for which the prefactor $\tau^{-1} \Delta \omega /\left[\left(\tau^{\prime-1}-\Delta \omega\right)^{2}+\right.$ $\left.\left(\tau^{\prime-1}-\Delta \omega^{\prime}\right)^{2}\right]$ is small, which would suggest that $\tau^{-1} \cong \Delta \omega$. However, In the infrared combination band spectra shown in the body of the paper, 
the positions of the observed peaks seem more consistent with the exchange predictions then with the scheme suggested by ( 94 ).

The avallable evidence tends to indicate that the imaginary components of $\delta w$ and $\tau$ are in fact small, in which case (91) retains its usefulness. It should be noted, however, that both of the mechanisms (91) and (94) will be present to a greater or lesser extent in any given situation, and a choice between them depends on a knowledge or estimate of the parameters involved. It seems desirable, therefore, to retain the exchange model (91) whenever possible, owing to the clear physical interpretation of the parameters $\delta \omega$ and $\tau$. 
APPENDIX 3

\section{LISTINGS OF COMPUTER PROGRAMS}

Two sets of programs are listed here: the first set performs the numerical integrations and convolutions for calculating the picosecond probe signal as a function of delay (see Chapter 4). The second set calculates self-phase modulated spectra using Fourler Transforms (see Chapter 5).

Not listed in this appendix are the graphics programs which plot the calculated functions, since they are quite long and call a lot of library subroutines. The graphics routines were written to be generally useful without detailed knowledge of how they work. They are very Interactive and the programming techniques require no subtleties that need to be explained. Some brief discussion of these programs will be given below in the proper places, but two general points should be mentioned: 1) The plotting subroutines called by the main programs are contained in two diskette libraries, TCSLIB and PACLIB. Manuals describing the former, supplied by Tektronix, are avallable. PACLIB was written by this author, and documentation is available from me or from several members of the group. 2) When making any modifications to either the picosecond or self-phase modulation programs, be careful not to change the format of the files that are output to the disk, because this will give the graphics programs trouble when they try to read them. Except for this restriction, the graphics programs should be generally applicable to any kind of output data. Listing of the programs are avallable on disk files for anyone who needs them. 


\section{Picosecond Probe Signal Versus Delay Calculation}

Because of the length of the calculation and the restrictions imposed by the size of the LSI-11 memory, the calculation of the probe curves is broken down into 2 steps, each requiring a separate program. There is also a third program which calculates the standard deviation of a set of experimental points from a calculated curve, and a fourth program which plots everything. Also included in the listings is KMATCH, which was used to draw Figure 27.

MaMA: The "parent" program of the set is titled "MaMA." This performs the numerical integration of Equation (46) as described in the text of Chapter 4. The progran calculates an array $Q$ which represents the vibrational amplitude as a function of time. $Q$ is numerically integrated along the z-axis, and can be output to the disk at the beginning and end of the cell, and at any desired number of intermediate steps. The calculated $Q$ must be stored on the disk to be read subsequently by the convolution program.

VARIABLE AND ARRAY NAMES AND MEANINGS -

(all arrays represent functions of time)

NZ

- the number of steps along $z$ at which data is to be output. If $\mathrm{NZ}$ is set to 1 , data is output at the beginning $(z=0)$ and end of the cell only.

NOUT - the number of steps taken along $z$ in between outputs. The total number of steps along $z$ is N2*NOUT, usually 100 .

ZINC - the size of the Increment along $z$ in $\mathrm{cm}$. The total cell length is NZ*NOUT*ZINC.

GSS - the steady-state stimulated Raman gain in $\mathrm{cm} / \mathrm{MW}$.

GAMMA $\quad-1 / T_{2}$

$T(400) \quad-$ holds the values of the function $\exp (-\Gamma t)$ 
NT - number of time intervals used (usually 300)

TINM - the size of each time interval (usually $0.2 \mathrm{psec}$ )

To - the starting point of the calculation In time (usually -25 psec); in other words, the time integration runs from To to TO+NT+TINC (usually from -25 to +35 psec)

$E(400)$ - Initlally holds the laser pulse shape $E(t)$, later holds $|E(t)|^{2} \exp (\Gamma t)$ (GO TO 201 in the source listing to see where the change occurs)

G(400) - collects the calculated values of the new $Q$ as the RungeKutta procedure progresses. In other words, it is first used to hold $k_{1}$, then $k_{1}+2 k_{2}$ etc. (see Equation (51)). In the comment lines in the source listing, "FIRST POINT" means first term $k_{1}$ in the Runge-Kutta formula (51). $Q(400)$ - the vibrational amplitude AUX(400) - a work-space array used to hold various things. SUBROUTINES CALLED:

INPUT - must be written by the user, and linked to the main program through COMMON. This is called at the very beginning, and must store data in the COMMON block arrays and varlables NZ, ZINC, GSS, GAMMA, T, NT, TINC, TO, E, NOUT. At this point, E should contain the laser field $E(t)$. This routine also must open a file for output. In the sample INPUT routing listed below, the values of all of the necessary parameters are read by INPUT from a data file. This version of INPUT calls a file opening routine OPENIT, a listing of which is also provided.

OUTPUT - also linked through COMMON to the main program, this routine can be used to output any kind of data in any desired format. It is entered at the beginning of the numerical integration, and NZ 
additional times during the progress of the calculation.

QSF

- numerical integration routine, used to perform the time integral in Equation (46). The version of this subroutine supplied in the DEC scientific subroutine package does not work. It has been modified to perform correctly; the modifled source listing can be found in the disk file QSFWRK.FOR. This routine is not listed here.

BRUTE. The convolution of the functions $|Q|^{2}$ and $\left|E_{L}\right|^{2}$ is performed by brute force, using Equation (49) exactly as written. This program opens the file containing, at different steps along $z$, the function $Q$ calculated by MAMA. The use of BRUTE can choose from which step along $z$ to take Q. MAMA's subroutine INPUT also had the foresight to write $E(t)$ Into this same file, so BRUTE can get both of the functions it needs from the same place. The convolution is done on every fifth point only, by shifting $|Q|^{2}$ in time with respect to $|E|^{2}$, multiplying them together, and then doing the Integration by calling QSF (see above under MAMA). The integrated values are stored in the array RESULT(400), which is written onto the disk (In a new file) at the end. All other varlable definitions here are the same as MAMA.

FITIT. Calculates the standard deviation from a set of calculated curves for a file of experimental data. This program requires a set of calculated curves to be resident on a disk which is assigned the logical unit name ZZ: . (Use monitor ASSIGN statement, e.g., ASSIGN DXI: ZZ). The file names must consist of 3 letters and 2 numerals, and must be numbered sequentlally, e.g., ABCOO.DAT, ABCOI.DAT, ABCO2.DAT, etc. There must also be avallable a file containing the experimental data, with error bars (see listing of example file to see format). FITIT takes the log of each 
experimental point and subtracts from it the $10 \mathrm{~g}$ of the calculated function (obtained by interpolation), thus forming a set of $\mathrm{N}$ numbers, where $N$ is the number of experimental observations. A (optionally) weighted least squares is performed on this set of numbers, and the result is output. The value of the experimental zero delay and the amount of information output can be changed interactively. This program does not call any subroutines.

BUBBLE. Plots both experimental data files and the files output by BRUTE that contaln the convoluted probe curves. To use this program, type $R$ BUBBLE and the program responds with a "!". After the exclamation point, the user must type a sequence of one-letter codes which give instructions to the program. Up to 10 codes will be accepted at once, and after the execution of a string of commands the program again prompts with "!". The only way to exit this program is with $a^{\wedge} \mathrm{C}$. The program will always request any Information it needs, such as file names. The acceptable one letter soimands are:

X - reads and plots experimental data files, with error bars

c - reads and plots a file containing a calculated curve

$E$ - erases the graphics screen

S - shifts a calculated curve left or right (the program will ask by how much)

N - normalizes a calculated curve (shifts it up or down)

I - lists parameters that were initially input to MAMA

R - replots experimental points (used to replot the same data after an erase)

H - turns hard copy plotter on

0 - turns hard copy plotter off. 


\section{Calculating Phase-Modulated Spectra by Fourier Transforms}

The main program here is a general Fourler transform program TRANS. The source listing is liberally supplied with comment lines, so only some general remarks will be made here. There are tricks to handling the Fast Fourler I'ransform correctly, and a reading of Brigham's book (Reference 72) is almost a prerequisite for using this program correctly. The program takes an Input function, transforms it, and writes the transformed function into a disk file. This file can be subsequently read and plotted by the program FFTPLT, which is completely interactive and self-explanatory. The user of TRANS must provide two subroutines:

CURVE - must store the function to be transformed as a set of complex numbers; the real part goes in the array XR(2048), and the imaginary part goes in XI(2048). CURVE is linked to TRANS through COMMON.

USER - can be entered if desired just before the transform is taken. This subroutine can perform any desired manipulations, for example, multiplying by the nonlinear phase factor $\phi$, as in the sample CURVE and USER below.

The Fourier Transform subroutine FFT (which can be found in Brigham's book), and attendant other functions and subroutines are kept in a library file FFTLIB. To link the program, the user must type in the files containing CURVE and USER, along with TRANS and FFTLIB. To link the program whose listing is shown below, which was the program that calculated the curves show in Figure 32, type:

R LINK

DXI: ZINTH9 $=$ DXI:TRANS, ZINTH9,FFTLIB 


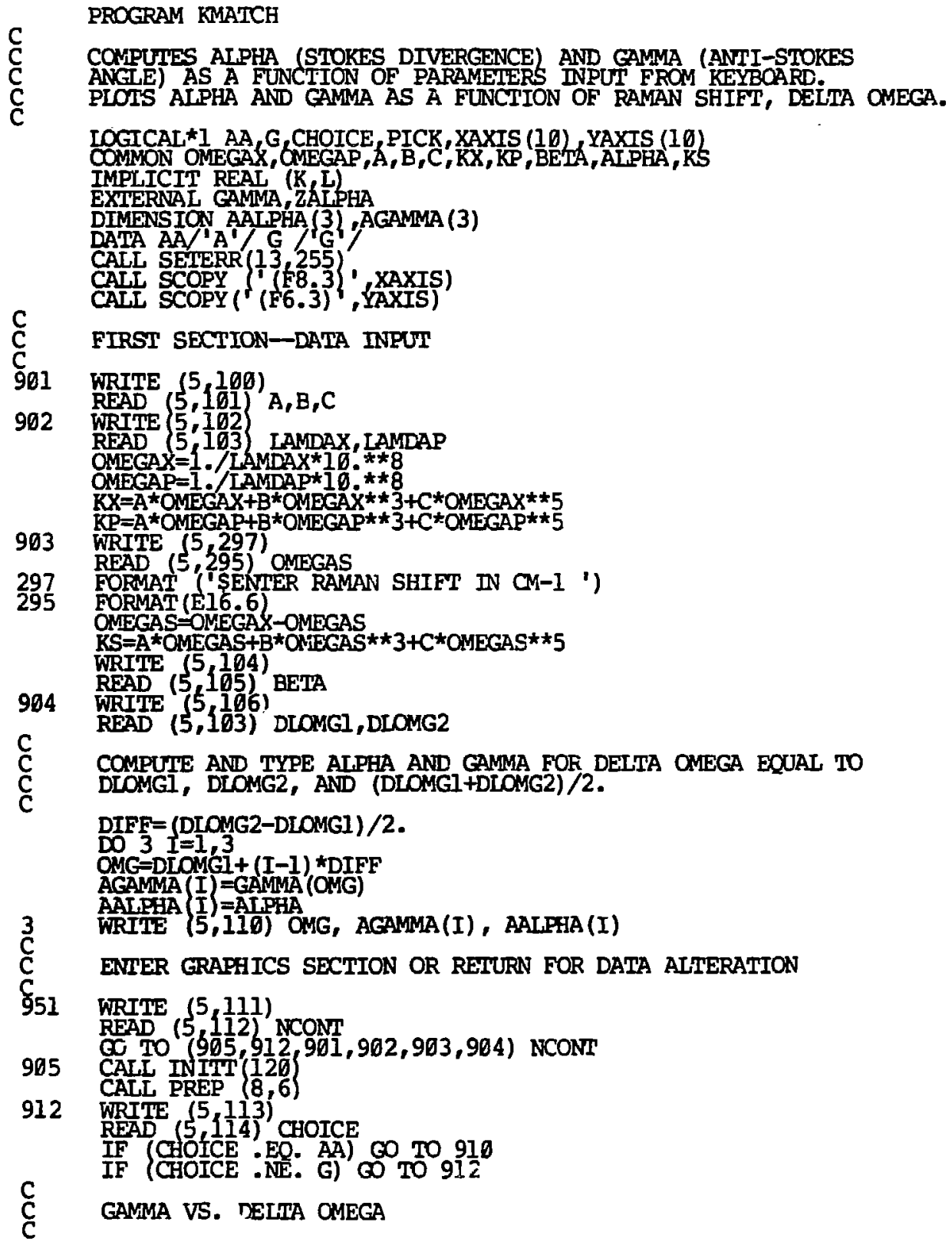


CALL DWINDO (DLOMG1, DLOMG2, AMINI (AGAMMA (1) ,AGAMMA (2) ,AGAMMA (3)), 1AMAXI (AGAMMA (1), AGAMMA (2), AGAMMA (3)))

CAIL WINADT $(X 1, \times 2, \times 3, \times 4)$

801 CALL FCNPLT (GAMMA, X1, X2)

CALL HICK $(6,6$, XAXIS, YAXIS $, 8,6)$

CALC HEADER

CALL HARD (J)

IF (J 9 EO. b) 60 TO 801

C

ALPHA VS. DELTPA CMEGA

910 CALL DWINDO (DLOMG1,DLOMG2,AMIN1 (AAL.PHA (1), AALPHA (2), AALPHA (3)), IAMAXI (AALLPAA (1), AAP. PHA 2 ), AALPFA (3)))

CALL WINADT $\times 1, \times 2, \times 3, \times 4)$

802 CALC FCNPLT (ZAT.PHA, X1, X2)

CALL HICK $(6,6$, XAXIS, YAXIS, 8,6$)$

CALL HEADER

CALL HARD (J)

IF (J.EO. D) $C O$ TO 862

GO TO 951

100

FORMAT

101

FORMAT (3E16.8)

FORMAT I SENITER EXCITING AND PROBE WAVELENGIHS (ANGSTROMS) : ")

FORMAT 2E16.8)

FORMAT 'SENTER BETA: ')

FORMAT EI6.8)

FORMAT ' DELTA OMEGA = , F9.3,' GAMMA = ',F8.6,' ALPHA= ',F8.6)

FORNAT (' ENIER CONIROL' INIEGER: '

1 1-PLOT WITH ERASE; 2-PLOT K'TTHOUT ERASE'/

1, 3-6: REENTER DATA STAPTING WTTH: $1 /$

1. 3-A, $B, C_{j}$ 4-WAVEIENGTHS; 5-BETA; 6-RAMAN SHIFT LIMITS. ')

$\frac{112}{113}$

FORMAT ' I 3

114 FORMAT $(1 \mathrm{~A})$

END

PROGRAM MAMA !STTMULATED RAMAN INTEGRATION

COMMN NZ, ZINC GSS, GAMMA, T, NT TTINC, TQ, E, G,, IZ, AUX, NOUT

C

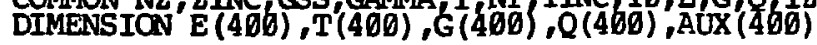

C SUBROUPINE 'INPUT' SUPPLIES THE VALUES OF THE PARAMETEFS

C NZ, ZINC, GSS, GAMMA, T,NI, TINC, T⿹, E

CALL INPUI

C LOP TO CALCULATE INITIAL CONDITIONS

DO $201 \quad I=1, \mathrm{NP}$

201

$Q\left(\frac{I}{T}\right)=E(I) / T(I)$

$\mathrm{E}\{\mathrm{I}\}=\mathrm{E}(\mathrm{I}) \neq \mathrm{E}\{\mathrm{I}\} / \mathrm{T}(\mathrm{I})$

CXI OSF (TIN , $Q, 0, N T$ )

DO $30 I I=1, N$

361

$Q(I)=T(I) * O(I)$

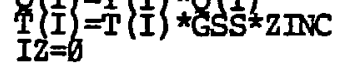




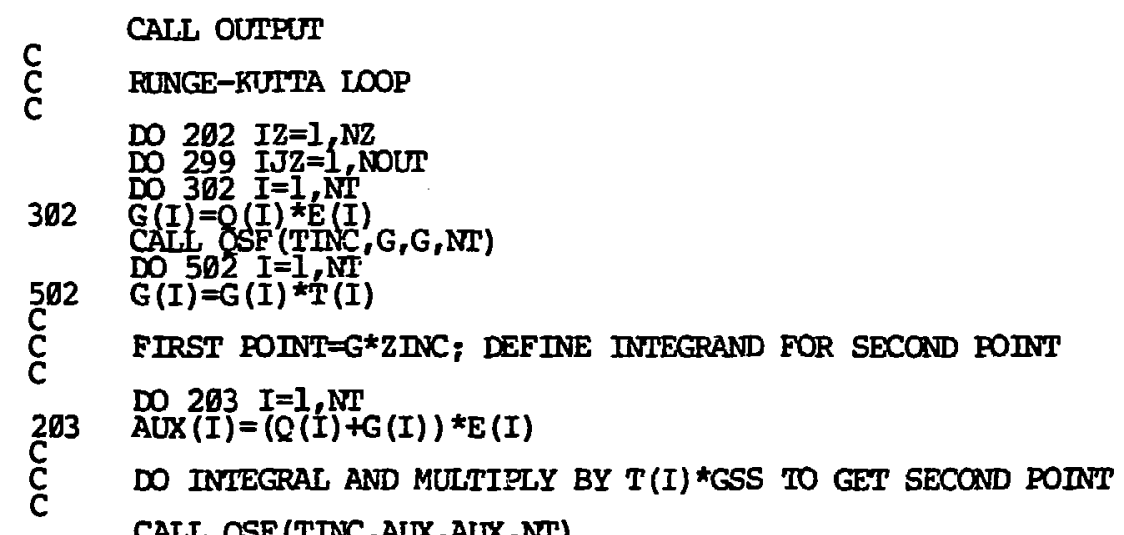

CAIL OSF (TINC, AUX, AUX, NT)

DO $303 \quad I=1$, NI

$303 \quad \begin{array}{ll}\mathrm{AUX}(I)=A U X \\ \mathrm{G}(\mathrm{I})=\mathrm{G}(\mathrm{I})+2 . * \mathrm{~T})\end{array}$

C

G(I) CONIATNS FIRST PT. + 2*SECOND POINT. CEFINE INIEGRAND FOR THIRD ROINT.

DO $204 \mathrm{I}=1, \mathrm{NT}$

$204 \quad \operatorname{AUX}(I)=(Q(I)+\mathrm{ALX}(I)) * E(I)$

C D INTEGRAL

CAL.L OSF (TINC, AUX, AUX, NI)

DO $304 \quad I=1, N$

$304 \quad \mathrm{G}(\mathrm{I})=G(\mathrm{I})+2 .{ }^{*} \mathrm{AUX}(\mathrm{I})$

C

G(I) NOW CONAATNS FIRST+2*SECORD+2*THIRD FOINIS.

C

C DO INIEGRAL AND CAICUIATE (I) VALUES FOR $Q(I)$

CAL OSF (TIK, AUX,AUX, NI)

DO $305 \quad I=1, N$

$\begin{array}{ll}395 & \mathrm{G}(\mathrm{I})=(\mathrm{G}(\mathrm{I})+\mathrm{A}(\mathrm{I}) \\ =\mathrm{G}(\mathrm{I})+\mathrm{Q}(\mathrm{I}) & \end{array}$

$(I) \star T(I)) \star .333333333$

C OUTFUI VALUES

299 CONTINUE

CALL OUTPUT

202 CONTINUE

STOP

END

SUBROUTINE INPUT IVERSION 3

COMMON NZ, ZTNC, GSS, GAMMA, T, NT, TTNC, TO, E, G, Q, IZ, AUX, NOUT

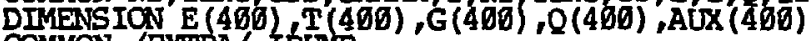
COMMON /EXTRA/IDLOME

C READS ALL INFORMATION FROM DATA FILE WITH USER-INPUT NAME. C SEIS UP OUTPUT FIIE STRUCTURE. 
100 FORME (5 ( 100$)$ FENIER NAME OF INPUT FILE..." )

CALL ASSIGN $(6, \mathrm{BOZO},-1)$

READ $(6,101)$ NZ, $2 \mathrm{MNC}^{-1}$, NOUT, GSS.PKI, $\mathrm{T} 2, \mathrm{NI}, \mathrm{TINC}, \mathrm{TV}, \mathrm{Cl}, \mathrm{C} 2$

101 FOLMAT (I5,E16.6, I5/3E16.6/I5, 2E16.6/2E16.6)

CALL OPENIT $(7, \mathrm{NZ}+3,2 \star \mathrm{NT}$, IDUMB,

WRITE (7'I) NZ,ZINC,NOUI,GSS,PKI,T2,NT,TMK, TO

GAMMA $=1 . / T 2$

GSS $=$ PKI $*$ GSS $\star 0.5 \star$ GAMMA

D $1, I=1, N$ I

TE $=T G+F I$ IAT $(I)$ *THNC

$T(I)=\operatorname{EXP}(-\mathrm{GAMMA} * \mathrm{TE})$

IF (TE -IE. G.) E (I $)=\operatorname{EXP}(-T E * T E / C l / C l * 0.5)$

IF TE TT:

$1 \quad$ COX I I $=E$

E(I)

WRITE (7'2) (AUX (I), I=1,NI)

REIURN

END

SUBROUTINE OUTPUT !VERSION 3

COMMON NZ, ZINC, GSS, GAMMA, T,NT,TINC, TD, E, G, Q, IZ, AUX

C THIS VERSION WRITES $Q * * 2$ ONIO THE DISK AT EACH STEP ALONG Z.

COMMON /EXIRA/IDUMB

DIMENSION E $(400), T(400), G(400), Q(400), A U X(400)$

$J=I Z+3$

DO $8 \mathrm{I}=1, \mathrm{NI}$

$G(I)=1 \cdot \bar{E}-5^{*} \mathrm{Q}(\mathrm{I}){ }^{\star} \mathrm{Q}(\mathrm{I})$

WRIIE $(7, J)$ \{G $(I), I=1, N r)$

REIURN

END

PROGRAM BRUTE

C CONVOLUTES PROBE PULSE SHAPE WITH VIBRATTONAL AMPITIUDE.

C INPUT DATA COMES FROM DATA FILE CFEATED BY PROGRAM 'MAMA'

1999 DIMENSION E (2000), Q (2000), X (2000), RESULT (40D)

$6 \quad \mathrm{E}(\mathrm{I})=0$.

CAI $=0$ ÖENIT $(6$, NIRAX, NRCRLS, IAV, 1$)$

READ $\left(6^{\prime} 1\right)$ NZ, ZINC, IOUT,GS, PKI,T2,NT, TINC, TO

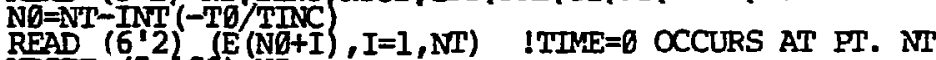
WRITE $(5,100)$ NZ

100 FORMAT ('SFIE CONIATNS', I4,' STEPS ALONG Z'/

1 'SCHOOSE DESIRED STEP NO...'।)

102 ROPMAT $\left(5, \frac{102)}{16}\right)$ IN

$\mathrm{IN}=\mathrm{IN}+2$

READ (6'TN) (Q (I), I=1,NT)

CALL CLOSE (6)

$\mathrm{N} \theta=\mathrm{N} T * 2-\mathrm{N} B$ 


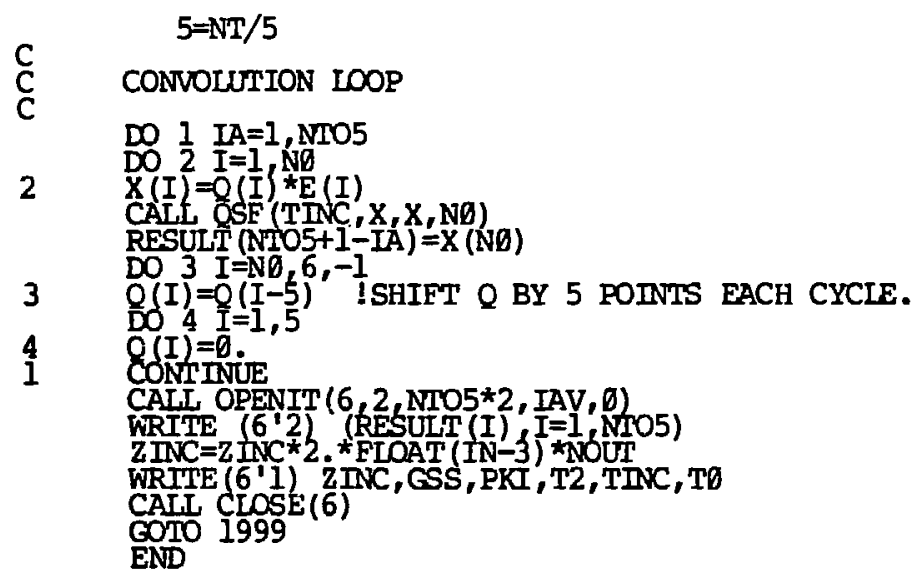

C STEPS THROUGH CURVES OF PROBE SIGNAL VS. DELAY AND C PERFORMS LEAST SOUARES COMPARISON WITH A SET OF

DIMENSION TTME (50) SIGNAL (50) ERROR (50) CALCUL (200) ,STIME (50)

DIMENSION TCALC (260) Z 5 (5) $X$ ( 50 ) IRAD (56)

LOGICAL‡1 NINE, ZERO, STOPZ (2), NAME (16), PREFIX (5), EXIEN (4)

DATA NINE, ZERO' 9 ' 6 '\%

EOUIVALENCE (PREFIX (1), NAME (4)), (EXIEN (1), NAME (9))

CALE SCOPY ('ZZ: ', NAME)

1 WRTTE $(5, *)$ 'Z2: 'WUST WE ASSIQNED'

CAIL ASSIGN $(6, \mathrm{BOZO},-1)$

WRITE $(5, *)$ 'ENIER OÚTFUT CODE'

C

READ $(5, *)$ ISUP

READ EXPERTMENTAL OBSERVATIONS

12 RFAD $(6, *$ END=112) STGNAL (I),STTME (I), ERROR (I)

ERROR II $\{=1 . /$ (AIOGIO SIGANAL (I) + ERROR (I) )-ALOGIO (SIGNAL (I))) **2 $\operatorname{SIGNAL}(\mathrm{I})=A \perp O G I 0$ (SIGNAL (I)) $\mathrm{I}=\mathrm{I}+1$

GOTO 12

112 NPTS $=I-1$

WRTTE $(5, *)$ 'ENTER ZERO OF DELAY, NO. OF STEPS, STEP SIZE' RFAD $(5 *$ ZERODI, NSTEPS, ZSTEP CALL CIOSE (6)

C SET UPTO READ CALCULATED FILES

WRITE $(5, *)$ 'STAPTING 3-LEITER FILENAME _2-DIGIT NO.' 
100 FORMAT (5, (5A0) PREFIX

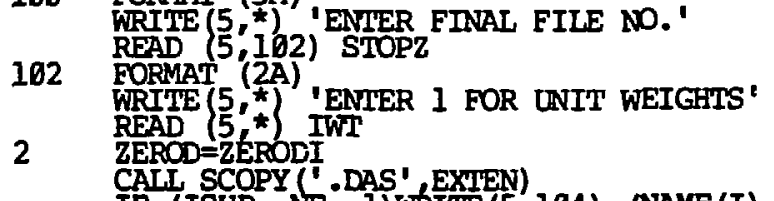

104 FORMAT (ISUP ACCESING FE $(5,104)$ (NAME (I) , I=1,8)

CALL ASSIGN $(6$, NAME, 12$)$

READ $(6,106)$ NTRAX, NKCRLS

106 FORMAT (2I8)

CALL CIOSE (6)

NAME (12) =NAME (12) +1

C C FOR FILE OPENING PROCEDURE; SEE IISTINC OF 'OPENIT'

CALL ASSTGN (6, NAME 12$)$

DEFTNE FILE 6 (NTRAX, NRCRDS, U, IAV)

READ $\left(6^{\prime}, 1\right)$ ZINC, GSS, PKI, T2, TINC, TV

$\mathrm{NT}=\mathrm{NRCRDS} / 2$

TINC $=$ TINC $* 5$.

READ $(6,2)$ jCALCUL (I), I=1,NT) !CALCULATED CURVE

CALL CLOSE (6)

DO $212 \mathrm{I}=1, \mathrm{NI}$

CALCULL(I) =ALOG10 (CALCUL(I))

212 TCALC $(I)=T Q+T M N * F L O A T$ (I)

C

INTERPOLATE BETWEEN NEAREST CALCULATED POINTS FOR EACH DATA FOINT

SIGMAM $=1 \cdot E+32$

DO 9000 IRS $=1$ NSTEPS

D $16 \mathrm{I}=1$, NPTS ICONVERT FROM MM. TO PSEC. DELAY

16 TMME $(I)=6.6666666^{*}$ (ZEROD-STIME (I) )

IBAES $=0$

DO 312 IBAD $I=1$, NPTS

$I 0=\emptyset$

412 IF 412 ICAIC $=2, N$ IX .GP. TTME(I) .AND. IQ .EQ. Ө) IQ=IX

IF IO

RATIO= (TIME (I)-TCAIC (IO-1)) ( TCALC (IQ) -TCAIC (IQ-1))

$Z(I)=C A I C U L(I Q-1)+R A T I O *$ (CAICUL (IQ) - CALCUL (IQ-I))

2012 IBAD $(I)=1$

IBAPS $=$ IBADS +1

$312 \mathrm{Z}(\mathrm{I})=\mathrm{I})=\mathrm{Z}(\mathrm{I})-\mathrm{SIGNAL}(\mathrm{I})$

C D WEIGHIED LEAST SQUARES

IF (IWT

$76 \quad$ ERROR $(\mathrm{I})=1$.

$66 \quad$ SUM $=0$.

SuMX $=\dot{\phi}$

SIGUA $=\dot{D}$

$D O 512 \quad \dot{I}=1$, NPTS 
IF (IBAD(I) EQ: 1) COTO 512

SUM=SUM+ERROR (I)

$512 \quad$ SUMX $=$ SUMX+ERROR
XMEAN=SUMX/SUM
DO 612 I $=1$ NPTS

612 SIGMA $=S I G M A+(Z(I)-X M E A N) * * 2$

SIGMA=SORT (SIGUA/FLOAT (NPTS-1-IBADS))

IF (SIGMA.LT. SIGMAM) SIGMAM=SIGMA

IF SIGMAM E. EQ SIGMA) ZERODM=ZEROD

IF ISUP EQ. I.OR. ISUP EO 2) GOTO 9000

WRITE $(5, *$ 'AUERAGE NORMAITIAATION' XMEAN 'STD.DEV.' SIGMA

WRITE $(5, *)$ 'Z=1,ZINC, T2=1,T2, ZERO DELAY', ZEROD

IF (ISUP' NE; O) 'COTO' $900 \overline{0}$

WRITE $(5, *)$ T RATIO TIME DIFF WEIGHT'

DO 712 I $=1$, NPTS

DIFE $=\mathrm{Z}(\mathrm{I})-\mathrm{XMEAN}$

IF (IBAD (I) $: E Q$. 1) $D I F F=0$.

71.2 CONTINUE

WRITE $(5, *) Z(I)$, TIME (I),DIFF, ERRRR (I)

9000 ZEROD=ZEROD+ZSTEP

8000 WRITE (5 *) 'T2= 'T2 'STD.DEV. ' SIGMAM, 'ZERO LELAY ' ZERODM IF (NAME (7). EQ. STOPZ (1) COAND. NAME (8) EQ. STOPZ (2)) COTOI NAME $(8)=\operatorname{NAME}(8)+1$.

1001 NAME $(7)=$ NAME $(7)+1$ NAME $\{8\}=Z E R O$

GOIO 2

END

SAMPLE BATCH COMMAND FILE LISTING:

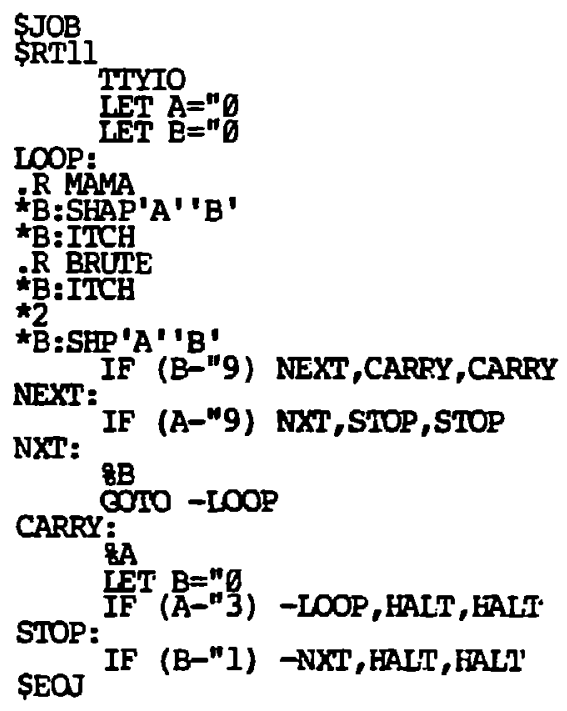


SAMPLE DATA FIIE FOR INPUT INIO 'MAMA':

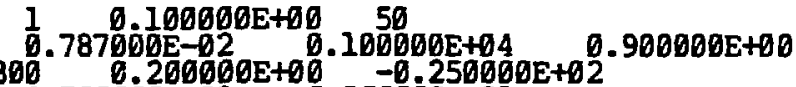

อ.700อ்

THIS PROGRAM PROVIDES A FLEXIBLE FOURIER TRANSFORM ROUTINE WITH SEVERAL INPUI AND OUTPUT OPTIONS. THE TRANSFORM IS ACCOMPLISHED BY THE SUBROUIINE 'FFT' WHICH IS TIAEN FROM 'THE FAST FOURIER TRANSEORM',E.O.BRIGHAM (PREMTICE-HALI, 1974) PAGE 164. TMPUT DATA TO BE TRANSFCRMED IS SLPPLIED EITHER THROUGH THE USER-WRITTEN SUBROUTINE 'CURVE' OR FROM A DISK FIIE. THE DATA EIIE MUST BE LNFORMATTED (SEE APPROPRIATE POINT IN SOURCE LISTING) . THIS DATA MAY BE FURTHER MANIPULATED BEFOFE TRANSFORMING BY TWO ADDITIONAL SURROUTINES:

1) 'USER' IS A USER-WRITIEN ROUTINE OPTIONALLY ENTERED BEFORE TRANSFORM IS TAKEN, WHICF CAN BE USED TO PERFORM ANY DESIRED OPERATIONS. 2) 'HANING' IS A SUPPLIED ROUTINE WHICH TREATS THE DATA USING THE 'EANNING TRUNCATION FUNCTION' METHOD AS DISCUSSED IN BRIGHAM CHAPIER 9. AFTER TRANSFORM IS COMPLETE, THE USER SELECTS THE FORM IN WHICH THE OUTPUT DATA IS IISPLAYED.

1) DATA CAN BE STORED AS REAL AND IMÄGINARY PART OF TRANSFORMED FINCTION, OVER THE INIERVAL $(0,2 P I)$. 2) DATA CAN BE STGEED AS AMPLITUDE AND PHASE FACTOR OVER THE INIERVAL (-PI,PI). AGAIN, SEE BRIGHAM CHA TIERS 2 AND 6. THIS PROGRAM IS DESIGND TO WORK ON ARRAYS GF 2048 POINTS. SNALIER ARRAYS ARE POSSIBIE, BUT THE ONLYY ALIONABLE NUMBER OF DATA POINIS IS 2* WILI HAVE AS MANY POINTS AS THE INPUT FUNCTION. AII SUBROUTINE LTNKAEE IS THROUEH COMMON.

XR-ARRAY CONTATNING THE REAL VALUES OF THE FLNCTION

XI-ARRAY CONAAINING THE IMAGINARY VALUES OF THE FUNCTION. M-INDICATES TOTAL NUMEER OF POINTS BEING USED BY TH REIATION N=2 $=$ M.

THE REIURAND TRANSFORMED FUNCTION IS STORED IN THE SAME ARRAYS XR AND XI; HENCE, INFUT DATA IS DESTROYED IN THE TRALSFCRM PROCESS.

THE COMPANION PROGRA, FFTPLT, CAN BE USED TO PLOT THE OUTPUT. CONRON XR(2048), XI (2048), M

DATA INFUT SECTION

$M=11$ !DEFAULT NUMBEF OF DATA POINTS IS 2048, UNLESS CHANGED WY 'CURVE' OR 'USER'.

100 FORMAT $\}$ IINPUT METHOD: 1 SURROUTINE, 0 DISK FIIE ')

102 RORMAT $(16)$ 


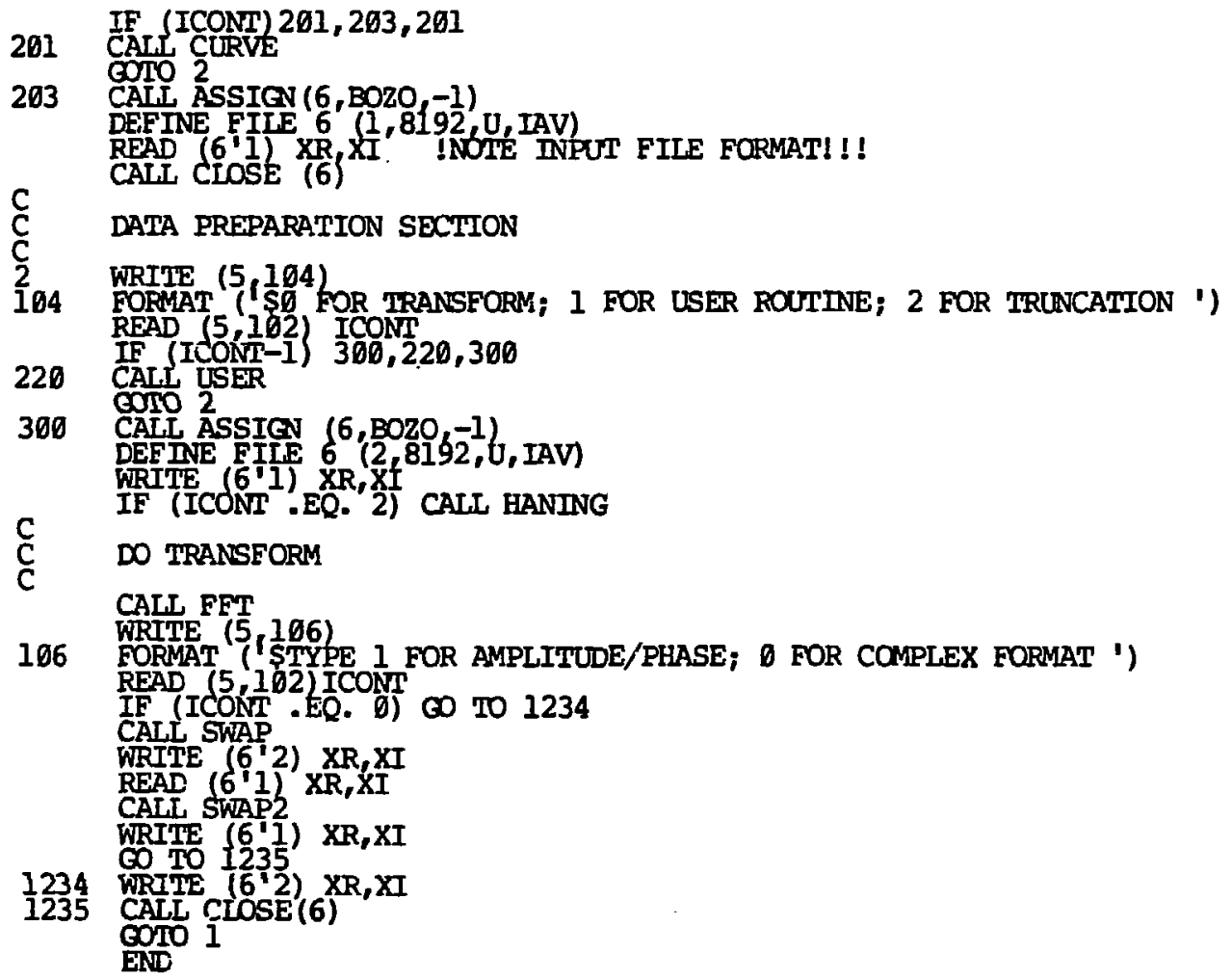
SUBROUTINE CURVE 1
STOKES PUISES
DATA PI $3.1415926 /$
REAL IBUEF COMMON XR (2048) XI (2048)

OONMON/BUFF/RGUTF (300), IBUFF (300), IZERO, NT DO $7 I=1,2048$

$\mathrm{XR}(\mathrm{I})=0$ :

703 XI I $=00$

1233 FOFMAT 'STYPE FIIENAME CONIAINING STOKES PULSE')

CALL ASSTGN (4,BOZO -1$)$

DEFINE FIIE 4 , 54,120 E, U IAV)

READ (4'1) NZ, INN NDUT, GSS, $\mathrm{KKI}, \mathrm{T} 2, \mathrm{NT}, \mathrm{TINC}, \mathrm{TV}$

IZERO=INT - TQ/TINC)

READ $(4$ N2 +3$)$ (RBUFF (I) $\operatorname{IBUFF}(I), I=1, N I)$

READ $(4,2)$ (IBUFF (I), I $=1, \mathrm{NI})$

CALL CLOSE (4) 
D DO 9 I I =, NT

DI010 FORMAT (2 (1PEI6.6))

9 RBUFF $(I)=R B U F F(I)$ IBUFF (I) WRITE $(5,1234)$

1234 FORMAT ( ENIER PERIOD IN POINTS (REAL NO.), INT. FACTOR; II NEW FIIE - 1 EXIT')

202 READ $(5,106)$ PER, FINT, LOOP

100 FORMAT (2E16.6, I6)

IF (PER EO. D.) PER=1.E+30

PER $=2 . \approx \mathrm{PI}$ PER

DO I I $=1$ NT

FHASE=FIOAT (I) *PER

INDEX $=1$ 025-IZERO+I

$1 \quad \mathrm{XIR}($ INDEX $)=$ FINT*RBUFF $(I) \star \operatorname{COS}($ PHASE $)+X R($ INDEX $)$

201 IF (LOOP) $201,202,203$

1235 FORMAT '\$ENIEE IASER INTENSITY FACTOR')

READ $(5,100)$ FINT

$2 \quad$ D 2 I=I,NI

$2 \operatorname{IBUEF}(I)=\operatorname{IBUFF}(I) * F T N T$

END

C SUBROUTINE USER ! ADDS PHASE FAC

C $\mathrm{C}$ OPT. COMM. 22,161 (1977)

COMPLEX Cl,C2

REAL IBUFF

COMMON XR (2048) XI (2048) M

COMMON/BUFF/RBUFF (300), IBUFF (300), IZERO, NI

1 WRITE $(5,100)$

100 FOFMAT 'SSENIER CONSTANT (CM**4/MN*2): ')

READ $(5,102)$ CONST

102 FORMAT $(\mathrm{E} 16.6)$

CONST=CONST*1.E-18

DO 7 I $=1,2048$

ICHECK $=I+I Z E R O-1025$

TEST=OVER (XI (I))

TEST=IEST+OVER (XR (I)

IF (ICHECK GP. $\varrho$ ALD. ICHECK . LT. 301) TEST=IEST+

IOVER (IBUFE (ICHECK))

TEST $=$ CONST $*$ TEST

IF (TEST LT

998 CI=CMPLX X X (I),XI (I) )

D IF (MOD $(I, 10)$.EO. O) WRITE $(5,110) \mathrm{Cl}, \mathrm{C} 2, \mathrm{I}, \mathrm{TEST}$

D110 FORMAT (4E14.6, I5,E14.6)

$7 \quad$ XR $(I)=$ REAL $(C I)$

$999 \quad$ C2 $=$ CMPLX (1 . TEST)

GOTO 998

END

FUNCTION OVER (A)

ISQUARES A WITHOUT DANGER OF OVER C INDERFLON

OVER $=0$

IF (ABS (A) . LT. 1.E-4)RETURN

IF (ABS A $)$.GT:1.E+14) REIURN

OVER $=A \star A$

RETURN 
END

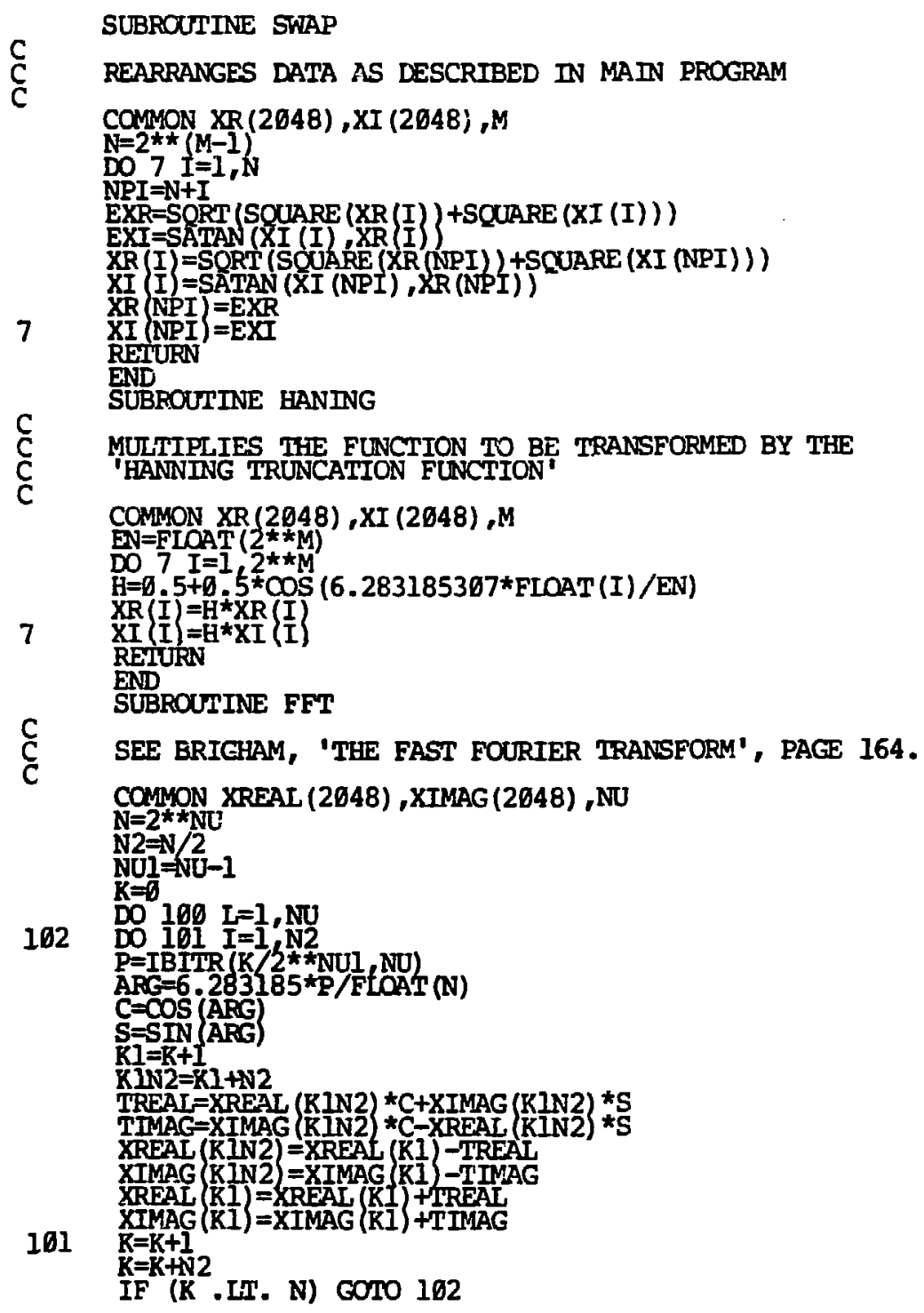




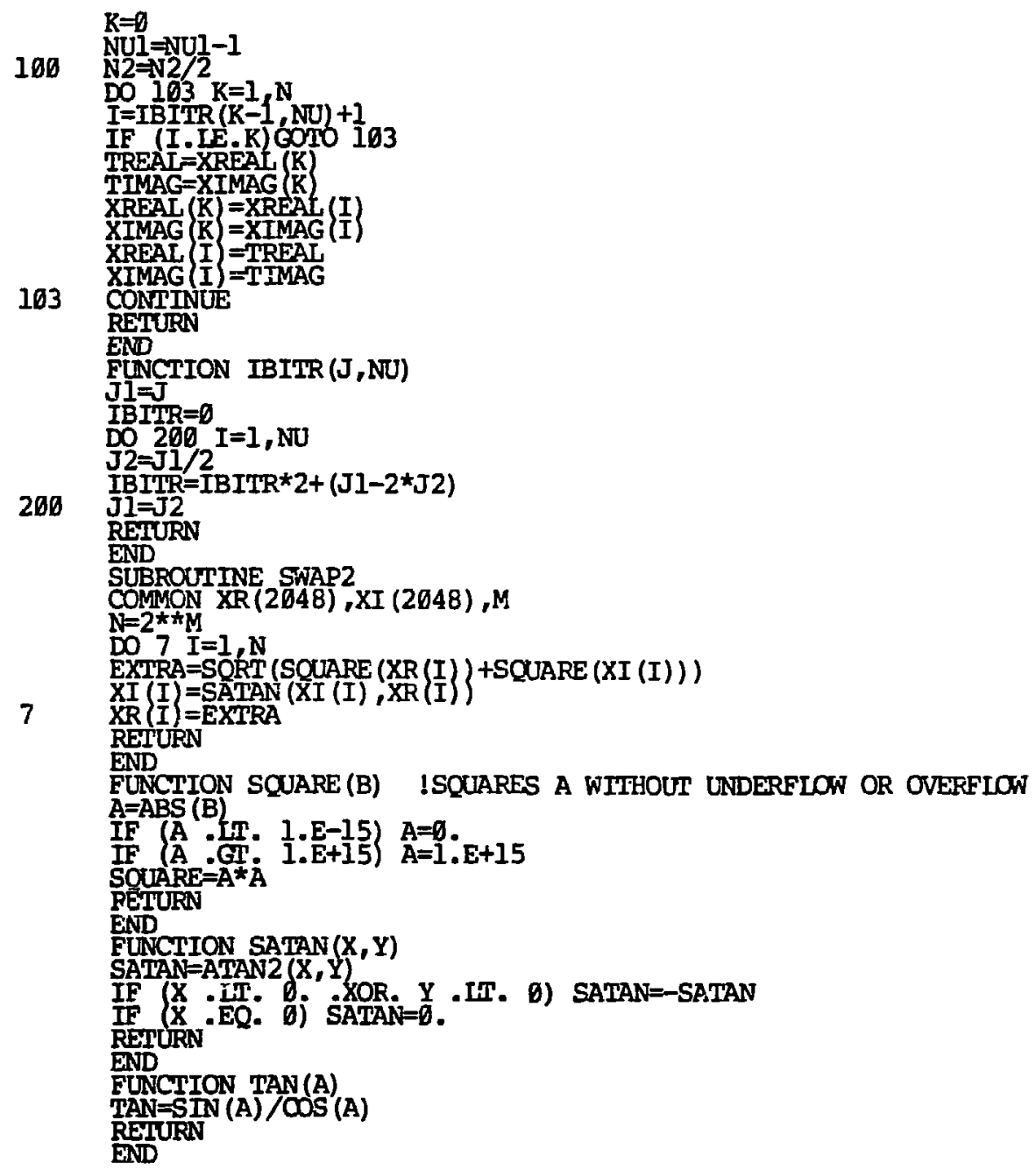




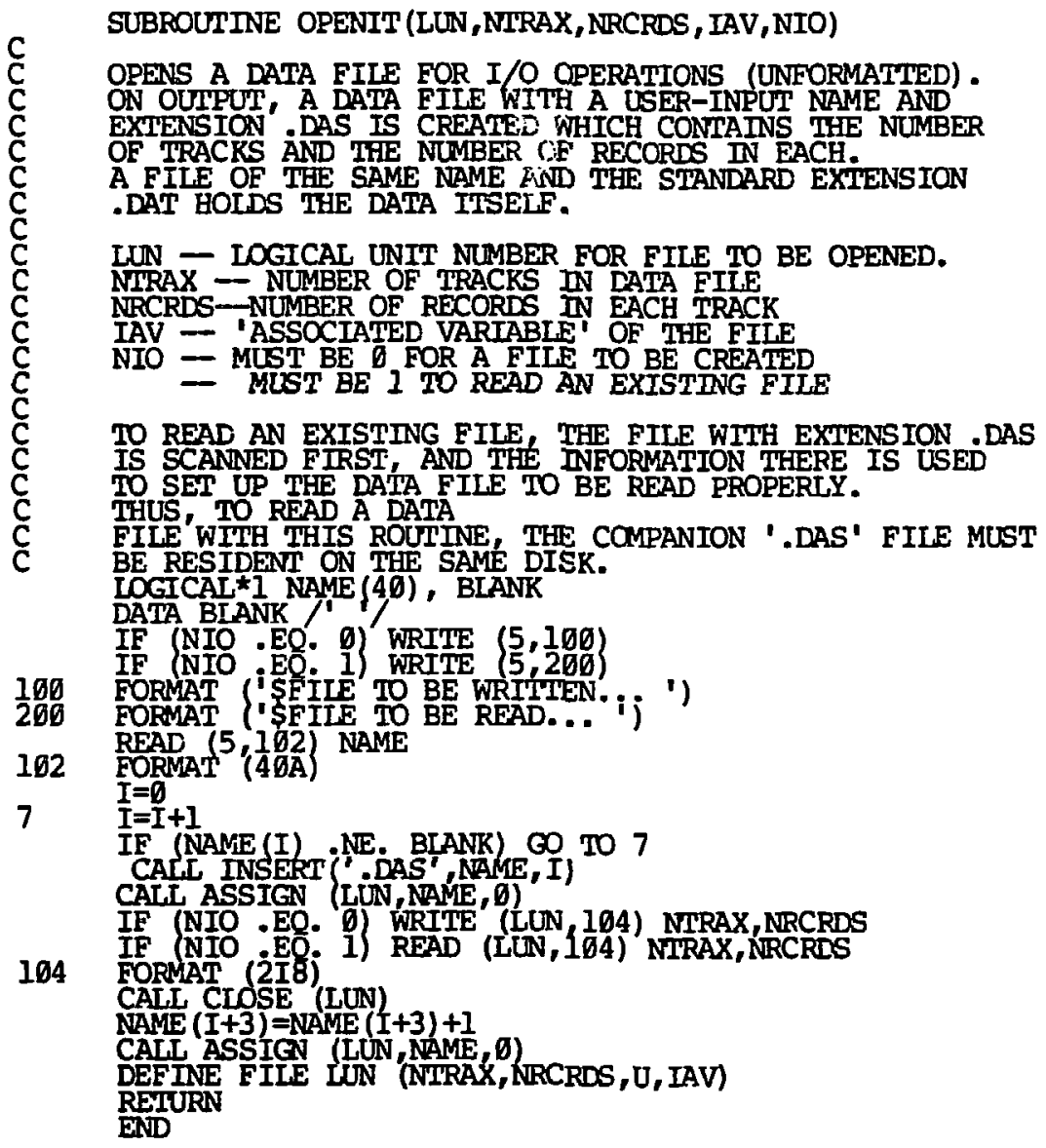




\section{REFERENCES}

1. D. von der Linde, A. Laubereau, and W. Ra1ser, Phys. Rev. Lett. 26, 954 (1971).

2. A. Laubereau, D. von der LInde, and W. Kaiser, Phys. Rev. Lett. 27, 802 (1971).

3. A. Laubereau, G. Wochner, and W. Kaiser, Opt. 14, 75 (1975).

4. A. Laubereau, D. von der Linde, and W. Kaiser, Phys. Rev. Lett. 28, 1162 (1972).

5. A. Laubereau, L. Kirschner, and W. Kaiser, Opt. Comm. 9, I (1973).

6. A. Laubereau, G. Wochner, and W. Kaiser, Phys. Rev. A13, 2212 (1976).

7. A. Laubereau and W. Kaiser, Chemlcal and Biochemical Applications of Lasers, C. B. Moore, ed. (Academic Press, 1976).

8. A. Laubereau and w. Kalser, Opto-electronics, 6, I (1974).

9. A. Laubereau and W. Kaiser, Rev. Mod. Phys. 50, 607 (1978).

10. C. B. Harris, P. A. Cornelius, and R. M. Shelby, in Advances in Laser Chemistry, A. H. Zewail, ed. (Springer Series in ChemicaI Physics), p. 223 (1978).

11. C. B. Harris, H. Auweter, and S. M. George, Phys. Rev. Lett. 44, 737 (1980).

12. R. M. Shelby, Ph.D. Thesis, University of California at Berkeley, 1978.

13. R. L. Carman, F. Shimizu, C. S. Wang, and N. Bloembergen, Phys. Rev. A2, 60 (1970).

14. R. L. Carman, M. E. Mack, F. Shimizu, and N. Bloembergen, Phys. Rev. Lett. 23, 1327 (1969).

15. C. B. Harris, R. M. Shelby, and P. A. Cornelius, Phys. Rev. Lett. 38, 1415 (1977).

16. C. B. Harris, R. M. Shelby, and P. A. Cornelius, Chem. Phys. Lett. 57, 8 (1978).

17. R. M. Shelby, C. B. Harris, and P. A. Cornelius, J. Chem. Phys. 70, 34 (1979).

18. R. K. Wertheimer, Chem. Phys. Lett. 52, 224 (1977).

19. R. K. Wertheimer, Mol. Phys. 38, 797 (1979). 
20. R. K. Wertheimer, Chem. Phys. 41, 229 (1979).

21. R. K. Wertheimer, Chem. Phys. 45, 415 (1980).

22. R. S. Abbott and D. W. Oxtoby, J. Chem. Phys. $\underline{70}, 4703$ (1979).

23. R. Kosloff and S. A. Rice, submitted to J. Chem: Phys.

24. G. J. Sma11, Chem. Phys. Lett. 57, 501 (1978).

25. S. Marks, P. A. Cornelius, and C. B. Harris, submitted to J. Chem. Phys.

26. C. A. van't Hof and J. Schmidt, Chem. Phys. Lett. 36, 460 (1975).

27. P. W. Anderson and P. R. Welss, Rev. Mod. Phys. 25, 269 (1953).

28. P. W. Anderson, J. Phys. Soc. Jap. 9, 888 (1954).

29. A. Nitzan and R. J. Silbey, J. Chem. Phys. 60, 407 (1974).

30. H. Mori, Prog. Theor. Phys. 33, 423 (1965).

31. P. N. Prasad and R. Kopelman, J. Chem. Phys. 58, 126 (1973).

32. P. N. Prasad and R. Kopelman, J. Chem. Phys. 58, 5031 (1973).

33. M. A. Kovner, Opt. Spektrosk. 1,348 (1956).

34. C. B. Harris and D. A. Zwemer, Ann. Rev. Phys. Chem. 29, 473 (1978).

35. Y. Toyozawa, Prog. Theor. Phys. 20, 53 (1958).

36. Y. Onodera and Y. Toyozawa, J. Phys. Soc. Jap. 24, 341 (1968).

37. J. C. Laufer and R. Kopelman, J. Chem. Phys. 53, 3674 (1970).

38, J. E. Cahill, J. Chem. Phys. 66, 4847 (1977).

39. H. M. McConne11, J. Chem. Phys. 28, 430 (1958).

40. R. Kubo and K. Tomita, J. Phys. Soc. Jap. , 888 (1954).

41. C. B. Harris, J. Chem. Phys. 67, 5607 (1977).

42. K. Dressler, 0. Oehler, and D. A. Smith, Phys. Rev. Lett. 34 , 1364 (1975).

43. J. Weisenfeld and C. B. Moore, Bull. Am. Phys. Soc. 21, 1289 (1976).

44. K. Tanabe and J. Jonas, J. Chem. Phys, 67, 4222 (1977).

45. S. F. Fischer and A. Laubereau, Chem. Phys. Lett. 35, 6 (1975). 
46. M. Shugard, J. C. Tully, and A. Nitzan, J. Chem. Phys. 69, 336 (1978).

47. S. Mukame1, Chem. Phys. $\underline{31}, 327$ (1978).

48. S. Mukamel, Chem. Phys. 37, 33 (1979).

49. R. Silbey, Ann. Rev. Phys. Chem. 27, 203 (1976).

50. D. W. Oxtoby and S. A. Rtce, Chem. Phys. Lett. 43, 1 (1976).

51. S. Voelker, R. M. Macfarlane, and J. H. Van der Waals, Chem. Phys. Lett. 53, 8 (1978).

52. M. N. Sapozhnikov, J. Chem. Phys. 68, 2352 (1978).

53. D. E. McCumber, Phys. Rev. 133A, 163 (1964).

54. A. G. Redfield, Adv. Mag. Res. Vol I, 1 (1965).

55. F. P. Burke and G. J. Sma11, J. Chem. Phys. 61, 4588 (1974).

56. S. Lin and H. Eyring, Proc. Natl. Acad. Sci. USA 74, 3623 (1977).

57. H. D. Ladouceur and D. J. Diestler, J. Chum. Phys. 70, 2620 (1979).

58. D. W. Oxtoby, J. Chem. Phys. 70, 2605 (1979).

59. D. W. Oxtoby, Adv. Chem. Phys. 40, 1 (1979).

60. M. Maier, W. Kalser, and J. A. Giordmane, Phys. Rev. 177, 580 (1969).

61. G. Arfken, Mathematical Methods for Physicists (Academic Press, 1970).

62. Data supplied by H. Auweter and S. M. George.

63. W. Zinth, A. Laubereau, and W. Raiser, Opt. Comm. 22, 161 (1977).

64. R. R. Alfano, L. L. Hope, and S. L. Shapiro, Phys. Rev. A6, 433 (1972).

65. R. H. Stolen and C. Len, Phys. Rev. A17, 1448 (1977).

66. T. K. Gustafson, J. P. Taran, H. A. Haus, J. R. Lifsitz, and P. L. Kelley, Phys. Rev. 177, 306 (1969).

67. F. Shimizu, Phys. Rev. Lett. 19, 1097 (1967).

68. E. Hecht and A. Zajac, Optics (Addison-Wesley, 1976).

69. N. Bloembergen, Nonlinear Optics (Benjamin, 1965).

70. G. Baldwin, Introduction to Nonlinear Optics (Plenum Press, 1969). 
71. Y. A. Cornelius and C. B. Harris, submitted to Opt. Coum.

72. E. 0. BrIgham, The Fast Fourler Transform (Prentice-Ha11, 1974).

73. C. K. Miller and J. F. Ward, Phys. Rev. A16, 1179 (1977); M. D. Levinson and N. Bloembergen, J. Chem. Phys. 60, 1323 (1973).

74. T. Kobayash1, Opt. Comm. 28, 147 (1979).

75. P. P. Ho and R. R. Alfano, Phys. Rev. A20, 2170 (1979). 